\title{
Testes Acelerados Assumindo um Modelo de Lei de Potência Inversa e Dados com Censuras do Tipo II
}

\author{
Teresa Cristina Martins Dias
}

Orientador: Dr. Jorge Alberto Achcar

Dissertação apresentada ao Instituto de Ciências Matemáticas de São Carlos USP, como parte dos requisitos para obtenção do título de mestre em "Ciências de Computação e Matemática Computacional".

São Carlos 
À minha familia 
Que triste seria o mundo

se tudo nele estivesse feito.

Se não se pudesse plantar uma flor

ou iniciar uma conquista.

Gabriela Mistral

Agradeço ao Prof. Dr. Jorge A. Achcar pela orientação segura e dedicada.

À Prof a . Maria Creusa S. Galvão Leite pelo incentivo no início do curso de pós - graduação.

Aos colegas e funcionários da UFSCar pela acolhida. Aos funcionários da USP pela colaboração na realização desse trabalho.

À CAPES pelo apoio financeiro parcial recebido.

A todos aqueles que de alguma maneira contribuiram para a realização desse trabalho. Em especial aos amigos Castilho, Silvely, Élvia, Gilberto e Joãozinho pela amizade e apoio durante este período, dentro e fora do ICMSC. 


\section{RESUMO}

Nesta dissertação de mestrado, alguns resultados sobre análise estatística de testes de sobrevivência acelerados são apresentados. Em particular, um modelo de teste de vida acelerado, Modelo de Lei de Potência Inversa, é considerado assumindo uma distribuição Exponencial para os tempos de sobrevivência dos componentes, com censuras do Tipo II. Análises clássica e Bayesiana são apresentadas para fazer inferências a respeito dos parâmetros de interesse. $\mathrm{Na}$ análise clássica, estimadores pontuais e intervalos de confiança são encontrados usando métodos assintóticos. Na análise Bayesiana, partindo de uma priori de Jeffreys, densidades a posteriori marginais para os parâmetros de interesse e intervalos de credibilidade são determinados, utilizando o Método de Laplace para aproximações de integrais. Uma análise Bayesiana é desenvolvida também, quando os tempos de falha seguem uma distribuição de Weibull. Também são apresentados, alguns métodos de Planejamento de Experimentos e uma aplicação de densidades preditivas em Controle de Qualidade.

Os métodos propostos são ilustrados através de dois exemplos. 


\begin{abstract}
In this dissertation, we present some results on the statistical analysis of accelerated life tests. In particular, we consider the Inverse Power Law Model, assuming an Exponential distribution for the lifetimes of the components with censored data under a type II censoring mechanism. Classical and Bayesian analysis are presented to get inferences about the parameters of interest. In the classical analysis, point estimates and confidence intervals are obtained using asymptotics methods. In the Bayesian analysis, assuming a noninformative Jeffreys prior density, marginal posterior densities for the parameters of interest and credible intervals are obtained using the Laplace's method for the approximation of integrals. Bayesian analysis is also considered when the failure times follow a Weibull distribution. Some methods for design of experiments are developed and an application of predictives densities in Quality Control is presented.
\end{abstract}

The proposed methods are illustrated with two numerical examples. 


\section{Conteúdo}

1 Introdução 1

2 Análise Clássica do Modelo de Lei de Potências 6

3 Análise Bayesiana 15

3.1 Análise Bayesiana assumindo $\alpha$ e $\beta$ desconhecidos . . . . . . . . . 16

3.2 Análise Bayesiana assumindo $\beta$ conhecido . . . . . . . . . . . 20

4 Planejamento de Experimentos $\quad 24$

4.1 Planejamento Seqüencial . . . . . . . . . . . . . . . 24

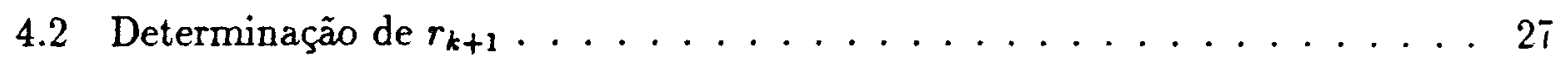

5 Densidades Preditivas $\quad 30$

5.1 Densidades Preditivas em Controle de Qualidade . . . . . . . . . . . . . 34

6 Análise Bayesiana assumindo uma Distribuição de Weibull para os tempos de sobrevivência $\quad 37$

6.1 Análise Bayesiana assumindo $\alpha, \beta$ e p desconhecidos . . . . . . . . 40

6.2 Posteriori Conjunta Marginal para $\theta_{1}^{*}$ e p: $\Pi\left(\theta_{1}^{*}, p \mid\right.$ dados $) \ldots \ldots . . . .44$ 
7 Dois Exemplos $\quad 48$

7.1 Um Exemplo com a Distribuição Exponencial . . . . . . . . . . . . . 48

7.2 Um Exemplo com a Distribuição de Weibull . . . . . . . . . . . . 57

7.2.1 Densidade a Posteriori Conjunta para $\theta_{1}^{*}$ e p: $\Pi\left(\theta_{1}^{*}, p \mid\right.$ dados $) \ldots . \quad 61$

8 Considerações Futuras $\quad 64$

Apêndice A Método Delta $\quad 66$

Apêndice B Aproximação de Integrais pelo Método de Laplace $\quad 68$

Apêndice C Densidade a Posteriori Marginal para $\delta_{1}=\ln \theta_{1}$ quando $\theta_{1}$ e $\beta$ são desconhecidos

Bibliografia 


\section{Capítulo 1}

\section{Introdução}

O estudo de confiabilidade de um componente ou de um sistema tem sido muito considerado na literatura estatística nas últimas décadas, por sua importância em sistemas de operação (eletrônicos, mecânicos, etc..). Quanto mais complexo for um sistema, mais difícil será achar uma medida de sua confiabilidade. Como um caso especial, considere os equipamentos eletrônicos atuais que são muito complexos devido ao grande avanço tecnológico e exigem componentes com baixa taxa de falha para se obter uma grande confiabilidade. Se o tipo de falha do sistema ou dos componentes é conhecido, pode-se então diminuir o custo da produção, garantir a manutenção e confiabilidade desse equipamento (ver por exemplo, Levenbach, 1957).

Determinar a taxa de falha em experimentos de teste de vida que são comuns na prática, é quase que impossível nas condições usuais de stress (temperatura, pressão, voltagem, ou outros fatores) e difíceis de serem simulados em laboratórios pois, normalmente exigem tempo ou custo muito elevados (ver por exemplo, Lawless, 1982).

Os testes acelerados consistem em observar o desempenho das peças submetidas a níveis de stress mais altos do que o usual, reduzindo artificialmente o tempo de falha dessas peças. A partir do conhecimento prévio a respeito da variação do comportamento de falha nas condiçóes usuais e através dos testes de sobrevivência conduzidos sob condiçōes aceleradas, chamados testes de sobrevivência acelerados, pode-se fazer inferências sobre caracteristicas da distribuição de vida correspondente às condições normais de uso do componente.

Um exemplo interessante mostrado por R. L. Guild da indústria Sylvania, citado em Levenbach (1957) está relacionado ao tempo de queima de válvulas de rádio. 
Neste exemplo, a distribuição ajustada ao tempo de falha não muda quando as condiçôes do teste se tornam mais rigorosas, resultando em uma redução no tempo de queima na razão $30: 1$.

Para experimentos onde a distribuição do tempo de vida dos componentes é conhecida, boas estimativas para os parâmetros da distribuição podem ser obtidas, sem ter que esperar pela falha de todos os elementos da amostra (ver por exemplo, Epstein \& Sobel, 1953). Este procedimento é conhecido como esquema de censuras.

Em situações onde o interesse é analisar a distribuição do tempo de vida de lâmpadas, válvulas de rádios, rolamentos ou de capacitores dielétricos, verifica-se que a distribuição Exponencial é muito usada na prática, pois caracteriza a distribuição do tempo de vida dos componentes e representa a taxa de falha constante para os itens.

De um modo geral, o conhecimento físico mostra que freqüentemente pode existir uma ou mais relaçôes entre os parâmetros da distribuição de falha e as condiçôes usuais do teste. $\mathrm{O}$ uso destes modelos ou relações tem sido discutido na literatura, especialmente pelos autores que tratam de mecanismos que induzem às falhas.

Mann, Schaffer e Singpurwalla (1974), descrevem alguns modelos, baseados no conceito de mecânica estatística, tais como encrgia de ativação e teoria cinética. Por exemplo, o Modelo de Arrhenius,

$$
\lambda_{i}=\exp \left\{\alpha-\frac{\beta}{V_{i}}\right\}
$$

e o Modelo de Eyring,

$$
\lambda_{i}=V_{i} \exp \left\{\alpha-\frac{\beta}{V_{i}}\right\}
$$

têm sua maior aplicação em componentes eletrônicos, como por exemplo, semi - condutores. O Modelo de Lei de Potência Inversa,

$$
\theta_{i}=\frac{\alpha}{V_{i}^{\beta}}
$$

muito popular na indústria e engenharia, tem sua aplicação em capacitores dielétricos. Os parâmetros $\alpha$ e $\beta$ são desconhecidos e $\mathrm{V}$ é a variável stress assumindo vários níveis, $i=1,2, \ldots, k$. Para componentes onde os tempos de sobrevivência seguem uma distribuição Exponencial, eles analisam cada uma dessas relações entre o parâmetro da distribuição de vida e a variável stress $V$. Estimadores de máxima verossimilhança foram encontrados para $\alpha, \beta$ e para o parâmetro de interesse $\lambda_{u}$ ou $\theta_{u}$ (tempo médio de sobrevivência dos itens nas condições normais de funcionamento). 
Klein \& Basu (1981) estudaram um modelo geral para tempos de falha com distribuição de Weibull, considerando o parâmetro de forma comum para todos os niveis de stress e os esquemas de censura do Tipo I, Tipo II e censura progressiva. Este Modelo Geral inclui como caso particular, cada um dos modelos acima citados: Arrhenius, Eyring e Lei de Potência. Klein \& Basu (1981a) estudaram este mesmo problema, considerando o parâmetro de forma diferente para cada um dos níveis de stress. Nestes estudos, encontraram estimadores de máxima verossimilhança para os parâmetros do Modelo Geral e de interesse. Nelson (1972), assumindo tempos de falha com distribuição de Weibull e a relação dada pelo Modelo de Lei de Potência Inversa, apresenta alguns métodos gráficos para verificar a adequabilidade do Modelo e estimar os parâmetros de interesse e percentis da distribuição de vida. Considerando uma relação linear inversa entre a variável stress e os parâmetros da distribuição Gaussiana Inversa, Bhattacharyya \& Fries (1981), determinam estimadores de máxima verossimilhança e estimadores de mínimos quadrados para os parâmetros de interesse.

Neste trabalho será desenvolvida uma análise clássica e Bayesiana assumindo que os tempos de falha dos componentes têm distribuição Exponencial, com função densidade,

$$
f\left(t, \lambda_{i}\right)=\lambda_{i} e^{-\lambda_{i} t}, \quad \lambda_{i}>0 \text { e } t \geq 0
$$

onde a relação entre o parâmetro da distribuição do tempo de sobrevivência, $\lambda_{i}$, e a variável stress para vários níveis, $V_{i}$, é dada pelo Modelo de Lei de Potência Inversa,

$$
\theta_{i}=\frac{\alpha}{V_{i}^{\beta}}
$$

onde $\alpha>0,-\infty<\beta<\infty$ e $\theta_{i}=\frac{1}{\lambda_{i}}$.

A adequabilidade do modelo Exponencial pode ser verificada usando métodos gráficos baseados em um estimador não - paramétrico da função de sobrevivência empírica, como por exemplo, o Estimador Produto - Limite de Kaplan \& Meier (1958).

Em geral os pesquisadores da área industrial e de engenharia justificam o uso deste modelo a partir de considerações empíricas (ver por exemplo, Levenbach, 1957).

Em situações práticas de teste de vida, os dados de sobrevivência, em geral, são incompletos ou censurados. Isto pode ser devido ao próprio planejamento do experimento considerado para a coleta dos dados.

Um plano amostral muito usado nesta área é dado pelo esquema de censuras de Tipo II, onde sob as condiçôes ambientais, o teste é aplicado nos componentes e termina 
quando um número pré - determinado de falhas é obtido. Além disso, são considerados $\mathrm{k}$ níveis de uma variável stress $V_{i}, i=1,2, \ldots, k$, assumindo uma distribuição Exponencial para os tempos de sobrevivência dos componentes e para cada nivel de stress é considerada uma amostra de $n_{i}$ unidades e $r_{i}$ falhas. Portanto, para um nivel $i$ de stress $V$, tem-se os tempos de sobrevivência ordenados associados a este nível $\mathrm{i}, t_{1 i}, t_{2 i}, \ldots, t_{r_{i} i}$ e $\left(n_{i}-r_{i}\right)$ observações censuradas iguais à $t_{r i i}, i=1,2, \ldots, k$.

No esquema de amostragem completa, todos os componentes da amostra são observados até que todos falhem. Para este plano amostral, as inferências são análogas ao esquema de censuras do Tipo II, quando $n=r$ ou $n_{i}=r_{i}$ para cada nível i de stress V.

Um outro esquema de censuras é dado pelo esquema de censuras de Tipo I. Nesse esquema, são observados os tempos de sobrevivència de $\mathrm{n}$ unidades durante um tempo fixo $t_{0}$. Este plano amostral tem grande aplicação na área médica. Lawless (1982), descreve outros planos amostrais.

Considerando os testes acelerados, é possível desenvolver métodos para planejar um experimento. $O$ pesquisador pode estar interessado em determinar um nivel adicional de stress $V_{i}$ ou ainda, determinar o número necessário de falhas $r_{i}$ para um dado nível de stress $V_{i}$. Zelen (1959), apresenta alguns métodos de planejamento de experimentos, envolvendo duas variáveis stress, assumindo as distribuições Exponencial com dois parâmetros, de Weibull e outras, para os tempos de vida dos componentes.

Um estudo importante dentro da Estatística está relacionado com a teoria de decisão e "predição" de observações futuras, considerando os dados observados . Na literatura, a aplicação de densidades preditivas tem sido de grande importância (ver por exemplo, Box \& Tiao, 1973; Aitchinson \& Dunsmore, 1975 e Press, 1988). Uma aplicação é dada por Achcar (1984) que determina a densidade preditiva para uma observação futura, para dados com censura do Tipo II, onde o logaritmo dos tempos de sobrevivência seguem um modelo Log - linear. Também verifica a adequabilidade do modelo proposto através de densidades preditivas. Achcar \& Bolfarine (1988), discutem alguns problemas de verificaçâo do modelo de regressão Gama Generalizado, usando densidades preditivas.

Em geral, o interesse do pesquisador está voltado para inferências sobre o tempo médio de vida dos componentes, sob condiçóes usuais de funcionamento. Numa análise clássica, os pesquisadores encontram os estimadores de máxima verossimilhança para os parâmetros $\alpha$ e $\beta$ e usam métodos assintóticos para as inferências sobre os parâmetros. Sprott \& Kalbfleish (1968), discutem e comparam resultados de funções de verossimilhança e aproximações da Teoria das Grandes Amostras, através de exemplos 
dados na literatura. Para o parâmetro $\theta_{1}$, tempo médio de vida sob as condições usuais de funcionamento, em geral, são considerados resultados assintóticos obtidos a partir do Método "Delta " (ver por exemplo, Miller, 1981).

Num estudo de testes acelerados, Bhattacharya (1967), apresenta uma análise Bayesiana do modelo Exponencial, discutindo o uso das densidades a priori Uniforme, Gama Inversa e a priori quasi - densidade.

O objetivo principal deste trabalho, é desenvolver uma análise Bayesiana do Modelo de Lei de Potência Inversa, assumindo uma priori de Jeffreys para os parâmetros $\alpha$ e $\beta$ (ver por exemplo, Box \& Tiao, 1973) e uma distribuição Exponencial para os tempos de vida dos componentes.

Em alguns casos, a taxa de falha dos componentes apresenta pequenas variações, podendo ser considerada constante, onde é possível ajustar a distribuição Exponencial para os dados. Neste trabalho, também será desenvolvida uma análise Bayesiana do Modelo de Lei de Potência Inversa assumindo uma distribuição de Weibull. Considerando a densidade a posteriori para o parâmetro de forma da distribuição de Weibull, pode-se verificar se a suposição de distribuição Exponencial é razoável para os dados pois, o modelo de Weibull engloba o modelo Exponencial como um caso particular.

Utilizando o Método de Laplace para aproximação de integrais, as densidades a posteriori marginais para os parâmetros de interesse são determinadas (ver por exemplo, Tierney \& Kadane, 1986; Tierney, Kass \& Kadane, 1989) quando não é possível resolver analiticamente as integrais envolvidas. Além disso, são desenvolvidos alguns métodos para planejamento de experimentos e uma aplicação de densidades preditivas em Controle de Qualidade é apresentada.

Os métodos propostos neste trabalho, são ilustrados com dois exemplos. 


\section{Capítulo 2}

\section{Análise Clássica do Modelo de Lei de Potências}

Neste capítulo será apresentada uma análise clássica para determinação de estimadores de máxima verossimilhança e intervalos de confiança para os parâmetros $\alpha$ e $\beta$ do Modelo de Lei de Potência, considerando que os dados provêm de uma amostra com distribuição Exponencial. Através desses estimadores, serão feitas inferências sobre características da distribuição de vida dos componentes sob condições usuais, em especial para o parâmetro de interesse $\theta_{1}$.

Considere um teste de sobrevivência acelerado em um planejamento onde são aplicados nos itens, $\mathrm{k}$ valores acelerados de stress $V_{i}, i=1,2, \ldots, k$. O comportamento de falha desses componentes é especificado pela distribuição Exponencial.

Seja então, $f\left(t ; \lambda_{i}\right)$ a função densidade de probabilidade da variável aleatória tempo de falha, $T>0$, dada por :

$$
f\left(t ; \lambda_{i}\right)= \begin{cases}\lambda_{i} e^{-\lambda_{i} t} & \lambda_{i}>0 \text { e } t \geq 0 \\ 0 & \text { caso contrário }\end{cases}
$$

Correspondente à função densidade de probabilidade, fica determinada a probabilidade acumulada de falha de um componente até o tempo $t$, dada pela função distribuição:

$$
F(t)=P[T \leq t]=\int_{0}^{t} f(x) d x
$$

e a probabilidade de que o componente não tenha falha até o tempo $t$, dada pela função 
de Sobrevivência ou função de Confiabilidade:

$$
S(t)=1-F(t)=P[T>t]=\int_{t}^{\infty} f(x) d x
$$

Considerando a função densidade de probabilidade, (2.1), tem-se:

$$
\begin{aligned}
& F(t)=\int_{0}^{t} \lambda e^{-\lambda x} d x=1-e^{-\lambda t} \\
& S(t)=e^{-\lambda t}
\end{aligned}
$$

Através da função Risco, $h(t)$, verifica-se que a função densidade Exponencial tem uma taxa constante de falha, $\lambda$ (ver por exemplo, Lawless, 1982):

$$
h(t)=\frac{f(t)}{S(t)}=\lambda
$$

Considerando $\mathrm{k}$ niveis de stress, tem-se que $\lambda_{i}$ é a taxa constante de falha sob um stress $V_{i}$ e $\theta_{i}=1 / \lambda_{i}$ é o tempo médio de sobrevivência sob o stress $V_{i}, i=1,2, \ldots, k$.

Assumir que a relação entre o parâmetro da distribuição de falha e o stress é definida pelo Modelo de Lei de Potência Inversa:

$$
\theta_{i}=\frac{\alpha}{V_{i}^{\beta}}
$$

para $\alpha$ e $\beta$ pertencentes ao espaço paramétrico $\theta$, onde $\theta=\{\alpha, \beta: \alpha>0 ;-\infty<\beta<\infty\}$.

Observar que $\alpha$ e $\beta$ são parâmetros desconhecidos a serem estimados.

Assumindo a relação (2.2) entre o parâmetro da distribuição de falha e o stress, duas suposições são feitas a respeito do comportamento de falha do planejamento:

(i) O aumento dos níveis de stress não muda o tipo da distribuição de sobrevivência, $f\left(t ; \lambda_{i}\right)$ da distribuição Exponencial, mas a variação dos niveis influencia os valores dos parâmetros;

(ii) A relação entre o stress e o parâmetro é válida somente para um certo intervalo de valores do stress. Fora desse intervalo, podem ocorrer alterações no mecanismo de falha do sistema, mudando essa relação (ver por exemplo, Singpurwalla, 1971).

Em testes de sobrevivência, os tempos de falha tornam-se disponiveis de uma maneira ordenada. Se n observações são submetidas ao mesmo estímulo de falha, então 
o item mais fraco falha primeiro, o segundo mais fraco é o próximo a falhar, e assim sucessivamente, até que o último item falhe.

Epstein \& Sobel (1953) mostram duas vantagens associadas à possibilidade de se interromper o teste antes de todas as $n$ observações falharem. A primeira diz respeito ao fato que se pode chegar a uma conclusão em menor tempo e com um número menor de observações a serem testadas e a segunda, que se pode obter um estimador não viciado e de variância mínima, para o parâmetro em estudo.

O esquema de censura do Tipo II adotado no planejamento, em tais situações, garante ainda uma economia no custo do experimento.

Assim, no inicio do teste, fixar o seguinte:

- k niveis de stress $V_{i}$ suficientemente grandes para induzirem falhas;

- $n_{i}$ itens a serem observados em cada nivel de stress;

- $r_{i}$ falhas correspondentes a cada nível $V_{i}$.

Os $\mathbf{k}$ testes são realizados aplicando-se constantemente o stress $V_{i}$ nas $n_{i}$ unidades. Interromper os testes logo após a $r_{i}$-ésima falha ocorrer e tomar os seus respectivos tempos de falhas aleatórias ordenados e não-censurados $t_{1 i}, t_{2 i}, \ldots, t_{r_{i} i}$ observados $\mathrm{e}$ as $\left(n_{i}-r_{i}\right)$ observações censuradas restantes iguais à $t_{r_{i} i}, i=1,2, \ldots, k$.

Assumir então que os tempos de falha constituem uma amostra de $n$ variáveis aleatórias independentes com a mesma distribuição, porém com o parâmetro $\lambda_{i}$ diferente para cada nivel de stress $V_{i}$ e desconhecido, onde a forma da função densidade é conhecida, dada por $(2.1)$ e $n=n_{1}+\cdots+n_{k}$. Tem-se por objetivo encontrar estatísticas, funções das observações $\left(t_{1 i}, t_{2 i}, \ldots, t_{r_{i} i}\right)$, para serem usadas como estimadores de $\alpha$ e $\beta$ ou de funções desses parâmetros tal como o modelo dado em (2.2).

Sendo $V_{1}$ o nivel de stress sob as condições usuais de funcionamento do teste, em particular, quer-se estimar o tempo médio de sobrevivência $\theta_{1}$, sob o nivel $V_{1}$.

Definição 2.1 Sejam $t_{(1 i)}<t_{(2 i)}<\ldots<t_{\left(r_{i} i\right)}$, os tempos observados das $r_{i}$ primeiras falhas das variáveis aleatórias independentes $T_{1 i}, T_{2 i}, \ldots, T_{n_{i} i}$ com função densidade dada em (2.1), $r_{i} \leq n_{i}$ produzidos sob um stress $V_{i} ;\left(t_{(1 i)}, t_{(2 i)}, \ldots, t_{\left(r_{i}\right)}\right)$ é chamado estatistica de ordem da amostra $i, i=1,2, \ldots, k$. 
Teorema 2.1 A função densidade conjunta das $r$ primeiras estatísticas de ordem de $\left(T_{(1 i)}, T_{(2 i)}, \ldots, T_{\left(n_{i} i\right)}\right)$ é dada por:

$$
f\left(t_{(1 i)}, \ldots, t_{\left(r_{i}\right)}\right)=\frac{n_{i} !}{\left(n_{i}-r_{i}\right) !} f\left(t_{(1 i)}\right) \ldots f\left(t_{\left(r_{i}\right)}\right)\left[S\left(t_{\left(r_{i}\right) ;} ; \lambda_{i}\right)\right]^{\left(n_{i}-r_{i}\right)}
$$

para $i=1,2, \ldots, k$ (ver por exemplo, Mood, Graybill \& Boes, 1974).

Para verificar a expressão acima, note que o coeficiente combinatório representa o número de maneiras a escolher uma unidade correspondente a cada um dos $t_{(1 i)}, \ldots, t_{\left(r_{i} i\right)}$ e $\left(n_{i}-r_{i}\right)$ unidades para os tempos de falhas não observados. $O$ fator produto representa a densidade conjunta dos $r_{i}$ tempos de falhas observados, dado o parâmetro $\lambda_{i}$, e finalmentc, o último fator representa a probabilidade que $\left(n_{i}-r_{i}\right)$ unidades tenham duração de vida maior que $t_{\left(r_{i}\right)}$.

Notação: Por facilidade, adota-se a seguinte igualdade:

$$
\left\{\begin{array}{c}
t_{(1 i)}=t_{1 i} \\
t_{(2 i)}=t_{2 i} \\
\vdots \\
t_{\left(r_{i} i\right)}=t_{r_{i} i}
\end{array}\right.
$$

Tem-se agora um modelo estatístico e a relação entre o parâmetro deste modelo e o stress bem definidos. Torna-se possivel então, estimar os parâmetros da distribuição que geram os dados e o parâmetro de interesse.

O Método da Máxima Verossimilhança, permite estimar os parâmetros $\alpha$ e $\beta$, e conseqüentemente estimar $\theta_{i}$. Este método consiste em encontrar o valor dos parâmetros $\alpha$ e $\beta$, que com maior probabililidade, produzem os dados $\left(t_{1 i}, t_{2 i}, \ldots, t_{r_{i} i}\right), i=1,2, \ldots, k$.

Definição 2.2 A função de verossimilhança do resultado observado, sob o modelo (2.1): com censuras do Tipo II assumindo $k$ niveis aleatorizados da variável stress, é dada por:

$$
L(\alpha, \beta)=\prod_{i=1}^{k}\left\{f\left(t_{1 i}, t_{2 i}, \ldots, t_{r_{i}} ; \lambda_{i}\right)\right\}
$$

onde $f\left(t_{1 i}, \ldots, t_{r_{i} i}\right)$ é dada em (2.3). 
Substituindo (2.3) na equação (2.4) e considerando $V_{1}, V_{2}, \ldots, V_{k}$ valores fixos, $L(\alpha, \beta)$ é dada por:

$$
\begin{aligned}
L(\alpha, \beta) & \propto \prod_{i=1}^{k}\left\{\prod_{j=1}^{r_{i}} f\left(t_{j i} ; \lambda_{i}\right)\left[1-F\left(t_{r_{i} i} ; \lambda_{i}\right)\right]^{\left(n_{i}-r_{i}\right)}\right\} \\
& \propto \prod_{i=1}^{k}\left\{\prod_{j=1}^{r_{i}} \lambda_{i} \exp \left[-\lambda_{i} t_{j i}\right]\left(\exp \left[-\lambda_{i} t_{r_{i} i}\right]\right)^{\left(n_{i}-r_{i}\right)}\right\}
\end{aligned}
$$

Como $\lambda_{i}=1 / \theta_{i}=V_{i}^{\beta} / \alpha$

$$
\begin{aligned}
L(\alpha, \beta) & \propto \prod_{i=1}^{k}\left\{\left(\prod_{j=1}^{r_{i}} \frac{V_{i}^{\beta}}{\alpha} \exp \left[-\frac{V_{i}^{\beta}}{\alpha} t_{j i}\right]\right)\left(\exp \left[-\frac{V_{i}^{\beta}}{\alpha} t_{r_{i} i}\right]\right)^{\left(n_{i}-r_{i}\right)}\right\} \\
& \propto \prod_{i=1}^{k}\left\{\left(\frac{V_{i}^{r_{i} \beta}}{\alpha^{r_{i}}} \exp \left[-\frac{\sum_{j=1}^{r_{i}} V_{i}^{\beta} t_{j i}}{\alpha}\right]\right)\left(\exp \left[-\frac{\left(n_{i}-r_{i}\right) V_{i}^{\beta} t_{r_{i} i}}{\alpha}\right]\right)\right\} \\
& \propto \prod_{i=1}^{k}\left\{\frac{V_{i}^{r_{i} \beta}}{\alpha^{r_{i}}} \exp \left\{-\frac{V_{i}^{\beta}}{\alpha}\left(\sum_{j=1}^{r_{i}} t_{j i}+\left(n_{i}-r_{i}\right) t_{r_{i} i}\right)\right]\right\}
\end{aligned}
$$

Definindo $A_{i}=\sum_{j=1}^{r_{i}} t_{j i}+\left(n_{i}-r_{i}\right) t_{r_{i} i}$, tem-se:

$$
L(\alpha, \beta) \propto \prod_{i=1}^{k}\left\{\frac{V_{i}^{\beta r_{i}}}{\alpha^{r_{i}}} \exp \left[-\frac{A_{i} V_{i}^{\beta}}{\alpha}\right]\right\}
$$

Suponha que exista um valor $(\hat{\alpha}, \hat{\beta})$ que maximize $L(\alpha, \beta)$. Então $(\hat{\alpha}, \widehat{\beta})$ é chamado de Estimador de Máxima Verossimilhança de $(\alpha, \beta)$. Em geral, o Estimador de Máxima Verossimilhança, quando existe, é um conceito muito usado por suas boas propriedades estatísticas (ver por exemplo, Bickel \& Doksum, 1977).

Resultado 2.1 Se $L(\alpha, \beta)$ dada em (2.4), é diferenciável em $\alpha$ e $\beta$, então $L(\alpha, \beta)$ e $l(\alpha, \beta)=\ln L(\alpha, \beta)$ possuem seus máximos para o mesmo valor de $(\alpha, \beta)$. Então $\max L(\alpha, \beta)=\max l(\alpha, \beta) ;$ portanto $(\hat{\alpha}, \widehat{\beta})$, os estimadores de máxima verossimilhança de $(\alpha, \beta)$, são soluções das equações:

$$
\left\{\begin{array}{l}
\frac{\partial l(\alpha, \beta)}{\partial \alpha}=0 \\
\frac{\partial l(\alpha, \beta)}{\partial \beta}=0
\end{array}\right.
$$


Portanto, aplicando o logaritmo em (2.5), $l(\alpha, \beta)$, é dado por:

$$
\begin{aligned}
l(\alpha, \beta) & =\ln L(\alpha, \beta) \\
& =\ln \left\{\prod_{i=1}^{k} \frac{V_{i}^{\beta r_{i}}}{\alpha^{r_{i}}} \exp \left[-A_{i} V_{i}^{\beta} / \alpha\right]\right\} \\
& =\sum_{i=1}^{k}\left\{\beta r_{i} \ln V_{i}-r_{i} \ln \alpha-\left(A_{i} V_{i}^{\beta}\right) / \alpha\right\} \\
& =\beta \sum_{i=1}^{k} r_{i} \ln V_{i}-\ln \alpha \sum_{i=1}^{k} r_{i}-\left(\sum_{i=1}^{k} A_{i} V_{i}^{\beta}\right) / \alpha
\end{aligned}
$$

Considerando todos os niveis i do stress $\mathrm{V}$, o número total de falhas observadas é dado por, $r=\sum_{i=1}^{k} r_{i}$. Então:

$$
l(\alpha, \beta)=\beta \sum_{i=1}^{k} r_{i} \ln V_{i}-r \ln \alpha-\frac{\sum_{i=1}^{k} A_{i} V_{i}^{\beta}}{\alpha}
$$

Os estimadores de máxima verossimilhança de $(\alpha, \beta)$ são dados pela resolução das equações:

$$
\left\{\begin{array} { l } 
{ \frac { \partial l ( \alpha , \beta ) } { \partial \alpha } = 0 } \\
{ \frac { \partial l ( \alpha , \beta ) } { \partial \beta } = 0 }
\end{array} \quad \text { então, } \quad \left\{\begin{array}{ll}
-r / \alpha+\left(\sum_{i=1}^{k} A_{i} V_{i}^{\beta}\right) / \alpha^{2} & =0 \\
\sum_{i=1}^{k} r_{i} \ln V_{i}-\left(\sum_{i=1}^{k} A_{i} V_{i}^{\beta} \ln V_{i}\right) / \alpha=0
\end{array}\right.\right.
$$

ou seja:

$$
\left\{\begin{array}{l}
\hat{\alpha}=\left(\sum_{i=1}^{k} A_{i} V_{i}^{\widehat{\beta}}\right) / r \\
\sum_{i=1}^{k} r_{i} \ln V_{i}=r \sum_{i=1}^{k} A_{i} V_{i}^{\widehat{\beta}} \ln V_{i} / \sum_{i=1}^{k} A_{i} V_{i}^{\widehat{\beta}}
\end{array}\right.
$$

onde $A_{i}=\sum_{j=1}^{r_{i}} t_{j i}+\left(n_{i}-r_{i}\right) t_{r_{i} i}$ e $i=1,2, \ldots, k$.

As equações de verossimilhança (2.7) são não lineares. Para solucionar numericamente estas equações, pode-se recorrer a métodos iterativos. Alguns artigos foram escritos com o propósito de analisar técnicas numéricas para soluções de equações de verossimilhança (ver Zacks, 1971). 
A partir dos Estimadores de Máxima Verossimilhança pontuais para $\alpha$ e $\beta$, intervalos de confiança podem ser obtidos para esses parâmetros, usando a Teoria das Grandes Amostras. Sob certas condiçōes, ela assegura que os Estimadores de Máxima Verossimilhança $\alpha$ e $\beta$ têm assintoticamente uma distribuição Normal Bivariada. É interessante observar que se a Matriz de Informação de Fisher é composta por constantes, os resultados assintóticos em geral são bem precisos, mesmo para amostras não muito grandes (ver por exemplo, Sprott, 1973, 1980). Para o caso estudado neste trabalho, é necessário muito cuidado em verificar a precisão, especialmente para amostras não muito grandes pois, a Matriz de Informação de Fisher contém termos envolvendo o parâmetro $\alpha$.

Suponha que uma função de verossimilhança $L(\alpha, \beta)$, de uma amostra aleatória de tamanho $\mathrm{n}$, seja diferenciável em $\alpha$ e $\beta$ até segunda ordem e se $(\ddot{\alpha}, \widehat{\beta})$ é Estimador de Máxima Verossimilhança para $(\alpha, \beta)$ então, $(\hat{\alpha}, \widehat{\beta})$ têm distribuiçãuo Normal assintótica. Assim, considere o seguinte resultado:

Resultado 2.2 Se a funçâo de verossimilhança (2.5) para $\alpha$ e $\beta$ é diferenciável em $(\alpha, \beta)$ até segunda ordem para um conjunto de dados fixado e se $(\hat{\alpha}, \widehat{\beta})$ é o Estimador de Máxima Verossimilhança de $(\alpha, \beta)$ para uma amostra aleatória de tamanho $n$, considerando $k$ niveis de stress, tem-se que:

$$
(\hat{\alpha}, \widehat{\beta}) \stackrel{a}{\sim} N\left\{(\alpha, \beta) ; I^{-1}(\widehat{\alpha}, \widehat{\beta})\right\}
$$

onde $I(\alpha, \beta)$ é a Matriz de Informação de Fisher, dada por:

$$
I(\alpha, \beta)=\left(\begin{array}{lr}
E\left\{-\partial^{2} l / \partial \alpha^{2}\right\} & E\left\{-\partial^{2} l / \partial \alpha \partial \beta\right\} \\
E\left\{-\partial^{2} l / \partial \beta \partial \alpha\right\} & E\left\{-\partial^{2} l / \partial \beta^{2}\right\}
\end{array}\right)
$$

Note que $I^{-1}(\widehat{\alpha}, \widehat{\beta})$, o inverso da Matriz de Informação de Fisher, é a Matriz de Variância e Covariância assintótica de $\hat{\alpha}$ e $\hat{\beta}$, dada por:

$$
I^{-1}(\hat{\alpha}, \hat{\beta})=\left(\begin{array}{cc}
\hat{\sigma}_{\alpha}^{2} & \hat{\sigma}_{\alpha \beta} \\
\hat{\sigma}_{\beta \alpha} & \hat{\sigma}_{\hat{\beta}}^{2}
\end{array}\right)
$$

(ver por exemplo, Bickel \& Doksum, 1977).

A partir da função de verossimilhança dada em (2.6) e das primeiras derivadas parciais dadas anteriormente em (2.7) e notando que $E\left\{A_{i}\right\}=\left(r_{i} \alpha\right) / V_{i}^{\beta}$, para todos os 
niveis de stress $i=1, \ldots, k$, tem-se que:

$$
\begin{gathered}
E\left\{-\frac{\partial^{2} l}{\partial \alpha^{2}}\right\}=-\frac{r}{\alpha^{2}}+\frac{2}{\alpha^{3}} \sum_{i=1}^{k} V_{i}^{\beta} \frac{r_{i} \alpha}{V_{i}^{\beta}}=\frac{r}{\alpha^{2}} \\
E\left\{-\frac{\partial^{2} l}{\partial \alpha \partial \beta}\right\}=E\left\{-\frac{\partial^{2} l}{\partial \beta \partial \alpha}\right\}=-\frac{1}{\alpha^{2}} \sum_{i=1}^{k} \frac{r_{i} \alpha}{V_{i}^{\beta}} V_{i}^{\beta}\left(\ln V_{i}\right)=-\frac{1}{\alpha} \sum_{i=1}^{k} r_{i}\left(\ln V_{i}\right) \\
E\left\{-\frac{\partial^{2} l}{\partial \beta^{2}}\right\}=\frac{1}{\alpha} \sum_{i=1}^{k} \frac{r_{i} \alpha}{V_{i}^{\beta}} V_{i}^{\beta}\left(\ln V_{i}\right)^{2}=\sum_{i=1}^{k} r_{i}\left(\ln V_{i}\right)^{2}
\end{gathered}
$$

Portanto, a Matriz de Informação de Fisher é dada por:

$$
I(\alpha, \beta)=\left(\begin{array}{cc}
r / \alpha^{2} & \left(-\sum_{i=1}^{k} r_{i} \ln V_{i}\right) / \alpha \\
\left(-\sum_{i=1}^{k} r_{i} \ln V_{i}\right) / \alpha & \sum_{i=1}^{k} r_{i}\left(\ln V_{i}\right)^{2}
\end{array}\right)
$$

Considerando a distribuição Normal Assintótica (2.8) para os Estimadores de Máxima Verossimilhança $\hat{\alpha}$ e $\hat{\beta}$, é possivel construir testes de hipóteses ou intervalos de confiança para os parâmetros $\alpha$ e $\beta$.

Assim, os intervalos de confiança para os parâmetros $\alpha$ e $\beta$, considerando um nivel de confiança $100(1-\gamma) \%$, são dados por:

$$
\begin{aligned}
& i c(\alpha)=\left[\hat{\alpha}-Z_{\gamma / 2} \widehat{\sigma}_{\alpha} ; \hat{\alpha}+Z_{\gamma / 2} \widehat{\sigma}_{\alpha}\right] \\
& i c(\beta)=\left[\hat{\beta}-Z_{\gamma / 2} \widehat{\sigma}_{\beta} ; \hat{\beta}+Z_{\gamma / 2} \hat{\sigma}_{\beta}\right]
\end{aligned}
$$

onde $Z_{\gamma / 2}$ é um percentil da distribuição Normal padronizada.

Note que o Estimador de Máxima Verossimilhança para $\theta_{1}=\alpha / V_{1}^{\beta}$ é dado por $\widehat{\theta}_{1}=\hat{\alpha} / V_{1}^{\widehat{\beta}}$. Para inferências sobre $\theta_{1}$, em geral os pesquisadores usam o Método Delta (ver Apêndice A). Como $\theta_{1}=g(\alpha, \beta)=\alpha / V_{1}^{\beta}$, tem-se,

$$
\left\{\begin{array}{l}
\frac{\partial g(\alpha, \beta)}{\partial \alpha}=1 / V_{1}^{\beta} \\
\frac{\partial g(\alpha, \beta)}{\partial \beta}=-\alpha \ln V_{1} / V_{1}^{\beta}
\end{array}\right.
$$


Portanto, $\hat{\theta}_{1}=\hat{\alpha} / V_{1}^{\hat{\beta}}$ tem uma distribuição Normal assintótica dada por,

$$
\hat{\theta}_{1} \stackrel{a}{\sim} N\left\{\frac{\alpha}{V_{1}^{\beta}} ; \hat{\sigma}_{\theta_{1}}^{2}\right\}
$$

onde

$$
\hat{\sigma}_{\theta_{1}}^{2}=\hat{\sigma}_{\alpha}^{2}\left(\frac{1}{V_{1}^{\widehat{\beta}}}\right)^{2}+2 \widehat{\sigma}_{\alpha \beta}\left(\frac{1}{V_{1}^{\widehat{\beta}}}\right)\left(\frac{\hat{\alpha} \ln V_{1}}{V_{1}^{\widehat{\beta}}}\right)+\hat{\sigma}_{\beta}^{2}\left(\frac{\widehat{\alpha} \ln V_{1}}{V_{1}^{\widehat{\beta}}}\right)^{2}
$$

e $\hat{\sigma}_{\alpha}^{2}, \hat{\sigma}_{\beta}^{2}$ e $\hat{\sigma}_{\alpha \beta}$ são dados em (2.9).

Portanto, um intervalo de confiança para $\theta_{1}$ com coeficiente de confiança $100(1-\gamma) \%$ é dado por:

$$
i c\left(\theta_{1}\right)=\left[\hat{\theta}_{1}-Z_{\gamma / 2} \hat{\sigma}_{\theta_{1}} ; \hat{\theta}_{1}+Z_{\gamma / 2} \hat{\sigma}_{\theta_{1}}\right]
$$

Em geral o pesquisador deve tomar muito cuidado no uso desses resultados assintóticos, especialmente para amostras pequenas ou moderadas. 


\section{Capítulo 3}

\section{Análise Bayesiana}

Neste capítulo será apresentada uma análise Bayesiana para o modelo Exponencial relacionado através de seu parâmetro $\lambda$, com o Modelo de Lei de Potência Inversa, propostos no Capítulo 2. Através de uma densidade a priori não - informativa de Jeffreys, será obtida a densidade a posteriori para os parâmetros $\alpha$ e $\beta$. Considerando inicialmente $\alpha$ e $\beta$ parâmetros desconhecidos, obtém-se a densidade a pesteriori conjunta para esses parâmetros e utilizando o Método de Laplace para aproximações de integrais, a densidade a posteriori para o parâmetro de interesse $\theta_{1}$ é obtida. Supondo $\beta$ conhecido, um intervalo de credibilidade é construído para $\theta_{1}$ numa forma muito simples.

Em Inferência Bayesiana, o parâmetro da distribuição de vida com vida média $\theta=1 / \lambda$ é considerado uma variável aleatória, com função densidade $\Pi(\theta)$, que expressa o conhecimento (ou ignorância) que se tem a respeito do verdadeiro valor desconhecido do parâmetro.

O Teorema de Bayes leva à determinação da densidade a posteriori do parâmetro condicionado aos dados observados, incorporando a informação adicional dada pela densidade a priori $\Pi(\theta)$ (ver Box \& Tiao, 1973). 


\subsection{Análise Bayesiana assumindo $\alpha$ e $\beta$ desconheci- dos}

Nesta secção, assuma que o pesquisador não tem qualquer conhecimento a priori da distribuição dos parâmetros $\alpha$ e $\beta$. O problema do estatístico Bayesiano consiste em encontrar uma densidade a priori conveniente, dado o espaço paramétrico de seus parâmetros. Uma escolha freqüente entre os Bayesianos é a priori não-informativa de Jeffreys. A escolha da priori não - informativa de Jeffreys é justificada por suas boas propriedades (ver Box \& Tiao, 1973).

Assumindo que o pesquisador não tem informação a respeito dos parâmetros $\alpha$ e $\beta$, a regra de Jeffreys determina uma densidade a priori não - informativa, dada por:

$$
\Pi(\alpha, \beta) \propto\{\operatorname{det} I(\alpha, \beta)\}^{1 / 2}
$$

onde $I(\alpha, \beta)$ é a Matriz de Informação de Fisher, descrita no Capítulo 2 .

Considerando a função densidade Exponencial (2.1), o Modelo de Lei de Potência (2.2) e o esquema de censuras do Tipo II descritos no Capítulo 2, a densidade a priori de Jeffreys para $\alpha$ e $\beta$ é dada por:

$$
\Pi(\alpha, \beta) \propto \frac{1}{\alpha}\left\{r \sum_{i=1}^{k} r_{i}\left(\ln V_{i}\right)^{2}-\left(\sum_{i=1}^{k} r_{i} \ln V_{i}\right)^{2}\right\}^{1 / 2}
$$

Como a expressão $\left\{r \sum_{i=1}^{k} r_{i}\left(\ln V_{i}\right)^{2}-\left(\sum_{i=1}^{k} r_{i} \ln V_{i}\right)^{2}\right\}^{1 / 2}$ é constante em relação à $\alpha$ e $\beta$, sem perda de informação, $\Pi(\alpha, \beta)$ pode ser dada por:

$$
\Pi(\alpha, \beta) \propto \frac{1}{\alpha} \quad \alpha \text { e } \beta \in \Theta
$$

onde $\Theta=\{(\alpha, \beta): \alpha>0$ e $-\infty<\beta<\infty\}$.

Especificada então $\Pi(\alpha, \beta)$, a densidade a posteriori conjunta para $\alpha$ e $\beta$ é determinada pelo Teorema de Bayes:

$$
\Pi(\alpha, \beta \mid \text { dados })=\frac{L(\alpha, \beta) \Pi(\alpha, \beta)}{\int_{-\infty}^{\infty} \int_{0}^{\infty} L(\alpha, \beta) \Pi(\alpha, \beta) d \alpha d \beta}
$$

onde $L(\alpha, \beta)$ é a função de verossimilhança (2.4) descrita no Capitulo 2 e $i=1,2, \ldots, k$. 
Observe que o conjunto de observações $\left(t_{1 i}, \ldots, t_{r_{i} i}\right)$ nesta etapa da análise, são dados que já foram observados, portanto não são mais considerados variáveis aleatórias. Este conjunto passa agora a ser denotado por dados.

Note que a integração do denominador da densidade $\Pi(\alpha, \beta \mid$ dados $)$ anterior, apresenta como resultado uma constante com respeito à $\alpha$ e $\beta$. Pelo Teorema de Bayes, se a função de verossimilhança for multiplicada por uma constante arbitrária, as inferências sobre a distribuição da densidade a posteriori não serão afetadas. Assim,

$$
\Pi(\alpha, \beta \mid \text { dados }) \propto L(\alpha, \beta) \Pi(\alpha, \beta)
$$

Substituindo $L(\alpha, \beta)$ e $\Pi(\alpha, \beta)$, respectivamente em (2.5) e (3.1), tem-se que:

$$
\begin{aligned}
\Pi(\alpha, \beta \mid \text { dados }) & \propto \frac{1}{\alpha}\left(\prod_{i=1}^{k} \frac{V_{i}^{\beta r_{i}}}{\alpha^{r_{i}}}\right) \exp \left\{\frac{-\sum_{i=1}^{k} A_{i} V_{i}^{\beta}}{\alpha}\right\} \\
& \propto \frac{1}{\alpha^{(r+1)}}\left(\prod_{i=1}^{k} V_{i}^{\beta r_{i}}\right) \exp \left\{\frac{-\sum_{i=1}^{k} A_{i} V_{i}^{\beta}}{\alpha}\right\}
\end{aligned}
$$

Portanto, a densidade a posteriori conjunta para $\alpha$ e $\beta$ é dada por:

$$
\Pi(\alpha, \beta \mid d a d o s) \propto \alpha^{-(r+1)}\left(\prod_{i=1}^{k} V_{i}^{\beta r_{i}}\right) \exp \left\{\frac{-\sum_{i=1}^{k} A_{i} V_{i}^{\beta}}{\alpha}\right\},
$$

para $i=1,2, \ldots, k$ e $\alpha$ e $\beta \in \Theta$.

A partir de (3.2), a densidade a posteriori marginal para $\beta$ é dada por:

$$
\begin{aligned}
\Pi(\beta \mid \text { dados }) & \propto \int_{0}^{\infty} \Pi(\alpha, \beta \mid \text { dados }) d \alpha \\
& \propto \int_{0}^{\infty} \alpha^{-(r+1)}\left(\prod_{i=1}^{k} V_{i}^{\beta r_{i}}\right) \exp \left\{\frac{-\sum_{i=1}^{k} A_{i} V_{i}^{\beta}}{\alpha}\right\} d \alpha \\
& \propto\left(\prod_{i=1}^{k} V_{i}^{\beta r_{i}}\right) \int_{0}^{\infty} \alpha^{-(r+1)} \exp \left\{\frac{-\sum_{i=1}^{k} A_{i} V_{i}^{\beta}}{\alpha}\right\} d \alpha
\end{aligned}
$$

\section{Resultado 3.1}

$$
\int_{0}^{\infty} b^{a} \theta^{-(a+1)} \exp \left[-\frac{b}{\theta}\right] d \theta=\Gamma(a)
$$


(ver por exemplo, Abramowitz \& Stegun, 1968).

Considerando,

$$
\left\{\begin{array}{l}
a=r \\
b=\sum_{i=1}^{k} A_{i} V_{i}^{\beta}
\end{array}\right.
$$

tem-se que:

$$
\Pi(\beta \mid \text { dados }) \propto \frac{\prod_{i=1}^{k} V_{i}^{\beta r_{i}}}{\left(\sum_{j=1}^{k} A_{j} V_{j}^{\beta}\right)^{r}} \Gamma(r)
$$

onde $\Gamma(r)=(r-1)$ !

Portanto, a densidade a posteriori marginal para $\beta$ é dada por:

$$
\Pi(\beta \mid \text { dados }) \propto \frac{\prod_{i=1}^{k} V_{i}^{\beta r_{i}}}{\left(\sum_{j=1}^{k} A_{j} V_{j}^{\beta}\right)^{r}}, \quad-\infty<\beta<\infty
$$

Através da maximização de (3.3), pode-se notar que o Estimador de Máxima Verossimilhança para $\beta$ coincide com o estimador da moda da densidade a posteriori marginal para $\beta$, usando a densidade a priori de Jeffreys (3.1):

$$
\ln \Pi(\beta \mid \text { dados }) \propto \beta \sum_{i=1}^{k} r_{i} \ln V_{i}-r \ln \left\{\sum_{i=1}^{k} A_{i} V_{i}^{\beta}\right\}
$$

Então:

$$
\left.\frac{d \ln \Pi(\beta \mid \text { dados })}{d \beta}\right|_{\beta=\bar{\beta}}=0, \quad \text { isto é, } \quad \sum_{i=1}^{k} r_{i} \ln V_{i}-r \frac{\left(\sum_{i=1}^{k} A_{i} V_{i}^{\beta} \ln V_{i}\right)}{\sum_{j=1}^{k} A_{j} V_{j}^{\beta}}=0
$$

Portanto, $\tilde{\beta}$, o estimador de $\beta$ dado pela moda a posteriori é dado por:

$$
r \frac{\sum_{i=1}^{k} A_{i} V_{i}^{\hat{\beta}} \ln V_{i}}{\sum_{j=1}^{k} A_{j} V_{j}^{\hat{\beta}}}=\sum_{i=1}^{k} r_{i} \ln V_{i}
$$

Usualmente, os objetivos dos pesquisadores e o interesse da indústria estão voltados para inferências a respeito de $\theta_{1}$, o tempo médio de vida dos componentes sob o nível de stress usual $V_{1}$. 
Considerando o modelo dado em (2.2), e a densidade a posteriori (3.2), uma técnica de transformação de variáveis é utilizada para determinação da densidade a posteriori conjunta para $\beta$ e $\theta_{1}$.

Seja então a transformação de variáveis:

$$
\left\{\begin{array}{l}
\beta=\beta \\
\theta_{1}=\alpha / V_{1}^{\beta} ; \quad \text { então, } \quad \alpha=\theta_{1} V_{1}^{\beta}
\end{array}\right.
$$

e a matriz Jacobiana dada por:

$$
J=\left(\begin{array}{ll}
\partial \beta / \partial \beta & \partial \beta / \partial \theta_{1} \\
\partial \alpha / \partial \beta & \partial \alpha / \partial \theta_{1}
\end{array}\right)
$$

ou seja:

$$
J=\left(\begin{array}{cc}
1 & 0 \\
\theta_{1} V_{1}^{\beta} \ln V_{1} & V_{1}^{\beta}
\end{array}\right)
$$

Portanto, $\operatorname{det}(J)=V_{1}^{\beta}$.

A densidade a posteriori conjunta para os parâmetros $\beta$ e $\theta_{1}$, é dada por:

$$
\Pi\left(\theta_{1}, \beta \mid \text { dados }\right) \propto \frac{\prod_{i=1}^{k} V_{i}^{\beta r_{i}}}{\left(\theta_{1} V_{1}^{\beta}\right)^{r+1}} \exp \left\{-\frac{\sum_{i=1}^{k} A_{i} V_{i}^{\beta}}{\theta_{1} V_{1}^{\beta}}\right\} V_{1}^{\beta}
$$

isto é,

$$
\Pi\left(\theta_{1}, \beta \mid \text { dados }\right) \propto \frac{\prod_{i=1}^{k} V_{i}^{\beta r_{i}}}{\left(\theta_{1}^{r+1}\right)\left(V_{1}^{\beta r}\right)} \exp \left\{-\frac{\sum_{i=1}^{k} A_{i} V_{i}^{\beta}}{\theta_{1} V_{1}^{\beta}}\right\}
$$

Integrando a densidade (3.5) em relação ao parâmetro $\beta$, obtém-se a densidade a posteriori marginal para $\theta_{1}$ :

$$
\Pi\left(\theta_{1} \mid \text { dados }\right) \propto \frac{1}{\theta_{1}^{r+1}} \int_{-\infty}^{\infty} \frac{\prod_{i=1}^{k} V_{i}^{\beta r_{i}}}{V_{1}^{\beta r}} \exp \left\{-\frac{\sum_{i=1}^{k} A_{i} V_{i}^{\beta}}{\theta_{1} V_{1}^{\beta}}\right\} d \beta
$$

Como existe dificuldade de se resolver analiticamente a integral em (3.6), um método para aproximação de integrais é usado. Tierney \& Kadane (1986) apresentam uma solução para este tipo de problema, dada pela aproximação de integrais pelo Método de Laplace. 
Pode-se reescrever a densidade (3.6) por,

$$
\Pi\left(\theta_{1} \mid \text { dados }\right) \propto \frac{1}{\theta_{1}^{r+1}} \int_{-\infty}^{\infty} \exp \left\{-n h_{\theta_{1}}(\beta)\right\} d \beta
$$

onde $n h_{\theta_{1}}(\beta)=\beta r \ln V_{1}-\beta \sum_{i=1}^{k} r_{i} \ln V_{i}+\left(\sum_{i=1}^{k} A_{i} V_{i}^{\beta}\right) / \theta_{1} V_{1}^{\beta}$

Se $\widehat{\beta}$ maximiza $-n h(\beta)$ e $\sigma=\left\{h^{\prime \prime}(\widehat{\beta})\right\}^{-1 / 2}$, então:

$$
\int \exp \{-n h(\beta)\} d \beta \approx \sqrt{2 \pi} \sigma n^{-1 / 2} \exp \{-n h(\widehat{\beta})\}
$$

(ver Apêndice B).

Então, a densidade a posteriori marginal para $\theta_{1}$ aproximada pelo Método de Laplace é dada por:

$$
\Pi\left(\theta_{1} \mid d a d o s\right) \propto \frac{\prod_{i=1}^{k} V_{i}^{\widehat{\beta} r}}{\theta_{1}^{(r-1 / 2)} V_{1}^{\widehat{\beta} r}}\left\{\sum_{i=1}^{k} A_{i}\left(\frac{V_{i}}{V_{1}}\right)^{\widehat{\beta}}\left(\ln \left(\frac{V_{i}}{V_{1}}\right)\right)^{2}\right\}^{-1 / 2} \exp \left\{-\frac{\sum_{i=1}^{k} A_{i} V_{i}^{\widehat{\beta}}}{\theta_{1} V_{1}^{\widehat{\beta}}}\right\}
$$

para $\theta_{1}>0$.

\subsection{Análise Bayesiana assumindo $\beta$ conhecido}

Assumindo agora que o parâmetro $\beta$ é conhecido, torna-se possível determinar um estimador pontual e construir um intervalo de credibilidade para o parâmetro de interesse $\theta_{1}$, numa forma bem simples.

em função de $\alpha$ :

A função de verossimilhança (2.5) para $\alpha$ e $\beta$, passa agora a ser dada somente

$$
L(\alpha) \propto \prod_{i=1}^{k} \frac{V_{i}^{\beta r_{i}}}{\alpha^{r_{i}}} \exp \left\{-\frac{A_{i} V_{i}^{\beta}}{\alpha}\right\}
$$

Considerando a densidade a priori de Jeffreys, $\Pi(\alpha) \propto 1 / \alpha, \alpha>0$, e a função de verossimilhança acima, a densidade a posteriori para $\alpha$ é dada por:

$$
\Pi(\alpha \mid d a d o s)=\frac{L(\alpha) \Pi(\alpha)}{\int_{0}^{\infty} L(\alpha) \Pi(\alpha) d \alpha}
$$


Isto é,

$$
\begin{aligned}
\Pi(\alpha \mid \text { dados }) & =\frac{\alpha^{-(r+1)} \prod_{i=1}^{k} V_{i}^{\beta r_{i}} \exp \left\{-\left(\sum_{i=1}^{k} A_{i} V_{i}^{\beta}\right) / \alpha\right\}}{\int_{0}^{\infty}\left[\Pi_{j=1}^{k} V_{j}^{\beta r_{j}} /\left(\alpha^{-(r+1)}\right)\right] \exp \left\{-\left(\sum_{j=1}^{k} A_{j} V_{j}^{\beta}\right) / \alpha\right\} d \alpha} \\
& =\frac{\alpha^{-(r+1)} \exp \left\{-\sum_{i=1}^{k} A_{i} V_{i}^{\beta} / \alpha\right\}}{\int_{0}^{\infty} \alpha^{-(r+1)} \exp \left\{-\sum_{j=1}^{k} A_{j} V_{j}^{\beta} / \alpha\right\} d \alpha}
\end{aligned}
$$

Definindo $b=\sum_{i=1}^{k} A_{i} V_{i}^{\beta}$ no Resultado (3.1), tem-se que a densidade a posteriori marginal para $\alpha$ é dada por:

$$
\Pi(\alpha \mid \text { dados })=\frac{\alpha^{-(r+1)}}{\Gamma(r)}\left\{\sum_{i=1}^{k} A_{i} V_{i}^{\beta}\right\}^{r} \exp \left\{-\frac{\sum_{i=1}^{k} A_{i} V_{i}^{\beta}}{\alpha}\right\}, \quad \alpha>0
$$

Considerando a densidade a posteriori para $\alpha$ dada em (3.8) e a transformação de variável $\theta_{1}=\alpha / V_{1}^{\beta}$, isto é, $\alpha=\theta_{1} V_{1}^{\beta}$, determina-se a densidade a posteriori para $\theta_{1}$, dada por:

$$
\Pi\left(\theta_{1} \mid d a d o s\right)=\frac{V_{1}^{\beta}}{\Gamma(r)}\left(\theta_{1} V_{1}^{\beta}\right)^{-(r+1)}\left(\sum_{i=1}^{k} A_{i} V_{i}^{\beta}\right)^{r} \exp \left\{-\frac{\sum_{i=1}^{k} A_{i} V_{i}^{\beta}}{\theta_{1} V_{1}^{\beta}}\right\}
$$

Logo, a densidade a posteriori para $\theta_{1}$, assumindo $\beta$ conhecido, é dada por:

$$
\Pi\left(\theta_{1} \mid \text { dados }\right)=\frac{\left(\sum_{i=1}^{k} A_{i} V_{i}^{\beta}\right)^{r}}{\Gamma(r) V_{1}^{\beta r} \theta_{1}^{(r+1)}} \exp \left\{-\frac{\sum_{i=1}^{k} A_{i} V_{i}^{\beta}}{\theta_{1} V_{1}^{\beta}}\right\}, \quad \theta_{1}>0
$$

A maximização da equação acima fornece um estimador pontual para $\theta_{1}$ : a moda a posteriori. O logaritmo de $\Pi\left(\theta_{1} \mid\right.$ dados $)$ é dado por,

$$
\ln \Pi\left(\theta_{1} \mid \text { dados }\right)=r \ln \sum_{i=1}^{k} A_{i} V_{i}^{\beta}-\ln \Gamma(r)-\beta r \ln V_{1}-(r+1) \ln \theta_{1}-\frac{\sum_{i=1}^{k} A_{i} V_{i}^{\beta}}{\theta_{1} V_{1}^{\beta}}
$$

$$
\operatorname{De},\left.\frac{d \ln \Pi\left(\theta_{1} \mid d a d o s\right)}{d \theta_{1}}\right|_{\theta_{1}=\tilde{\theta_{1}}}=0, \text { tem-se que, }-\frac{r+1}{\theta_{1}}-\frac{1}{V_{1}^{\beta}}\left[-\frac{\sum_{i=1}^{k} A_{i} V_{i}^{\beta}}{\theta_{1}^{2}}\right]=0
$$
é dado por:

Portanto, $\tilde{\theta}_{1}$, o estimador de $\theta_{1}$ dado pela moda a posteriori com $\beta$ conhecido

$$
\tilde{\theta}_{1}=\frac{\sum_{i=1}^{k} A_{i}\left(V_{i} / V_{1}\right)^{\beta}}{r+1}
$$


Uma maneira de resumir parcialmente as informações contidas na distribuição a posteriori é através de intervalos de credibilidade. Vários intervalos podem ser construídos para cada parâmetro de interesse. equação (3.9).

Como especificado anteriormente, a densidade a posteriori para $\theta_{1}$ é dada na

Definindo, $C=\left(\sum_{i=1}^{k} A_{i} V_{i}^{\beta}\right) / V_{1}^{\beta}$ tem-se:

$$
\Pi\left(\theta_{1} \mid \text { dados }\right)=\frac{C^{r}}{\theta_{1}^{(r+1)} \Gamma(r)} \exp \left\{-\frac{C}{\theta_{1}}\right\}
$$

Pela técnica da transformação de variáveis, verifica-se que a distribuição a posteriori para a variável $\lambda_{1}=1 / \theta_{1}$, com $\left|d \theta_{1} / d \lambda_{1}\right|=1 / \lambda_{1}^{2}$, é dada por:

$$
\begin{gathered}
\Pi\left(\lambda_{1} \mid \text { dados }\right)=\frac{C^{r}}{\lambda_{1}^{2} \Gamma(r)}\left\{\frac{1}{\lambda_{1}}\right\}^{-(r+1)} \exp \left\{-\frac{C}{\lambda_{1}^{-1}}\right\} . \\
\text { Isto é, } \Pi\left(\lambda_{1} \mid \text { dados }\right)=\frac{\lambda_{1}^{-(r+1)}}{\Gamma(r)} C^{r} \exp \left\{-\lambda_{1} C\right\}, \quad \lambda_{1}>O
\end{gathered}
$$

Note que $\quad \lambda_{1} \mid$ dados $\sim \operatorname{Gama}(r ; C)$

ou seja, a variável $\lambda_{1}$ condicionada aos dados observados tem distribuição Gama com parâmetros $C=\left(\sum_{i=1}^{k} A_{i} V_{i}^{\beta}\right) / V_{1}^{\beta}$ e $r=\sum_{i=1}^{k} r_{i}$ é um número inteiro positivo.

Através de uma nova transformação de variáveis, pode-se chegar a uma outra distribuição conhecida, porém com maior facilidade para se trabalhar na construção de intervalos de credibilidade. A distribuição $\chi^{2}$ é um caso particular da distribuição Gama (ver por exemplo, Mood, Graybill \& Boes, 1974).

Definindo, $C \lambda_{1}=x / 2$, isto é, $\lambda_{1}=x / 2 C$ onde $\left|d \lambda_{1} / d x\right|=1 / 2 C$, tem-se que,

$$
\begin{aligned}
& \Pi(x \mid \text { dados })=\frac{C^{r}}{\Gamma(r)}\left(\frac{x}{2 C}\right)^{r-1} \exp \left\{-C \frac{x}{2 C}\right\} \frac{1}{2 C} \\
& \text { Portanto, } \Pi(x \mid \text { dados })=\frac{C^{r}}{\Gamma(r)} \frac{x^{(r-1)}}{2^{r} C^{r}} \exp \left\{-\frac{x}{2}\right\}
\end{aligned}
$$


onde

$$
\left\{\begin{array}{l}
C=\left(\sum_{i=1}^{k} A_{i} V_{i}^{\beta}\right) / V_{1}^{\beta} \\
r=\sum_{i=1}^{k} r_{i} \\
X=2 C \lambda_{1}
\end{array}\right.
$$

Assim, a densidade a posteriori para a variável $\mathrm{X}$ condicionada aos dados tem uma distribuição $\chi^{2}$ com (2r) graus de liberdade:

$$
\Pi(x \mid \text { dados })=\frac{x^{(2 r / 2)-1}}{\Gamma(2 r / 2) 2^{2 r / 2}} \exp \left\{-\frac{x}{2}\right\}
$$

ou seja,

$$
X \mid \text { dados }=\frac{2 \sum_{i=1}^{k} A_{i} V_{i}^{\beta}}{V_{1}^{\beta} \theta_{1}} \sim \chi_{2 r}^{2}
$$

Um intervalo de credilidade para $\theta_{1}$ com probabilidade $(1-\gamma)$, é dado por:

$$
P\left\{\chi_{2 r}^{2}(\gamma / 2) \leq X \leq \chi_{2 r}^{2}(1-\gamma / 2)\right\}=1-\gamma
$$

isto é,

$$
P\left\{\chi_{2 r}^{2}(\gamma / 2) \leq \frac{2 \sum_{i=1}^{k} A_{i} V_{i}^{\beta}}{\theta_{1} V_{1}^{\beta}} \leq \chi_{2 r}^{2}(1-\gamma / 2)\right\}=1-\gamma
$$

onde $\chi_{2 r}^{2}(\gamma / 2)$ e $\chi_{2 r}^{2}(1-\gamma / 2)$ são percentis da distribuição Quiquadrado com $2 \mathrm{r}$ graus de liberdade, dados por: $P\left\{X \leq \chi_{2 r}^{2}(1-\gamma / 2)\right\}=1-\gamma / 2$ e $P\left\{X \leq \chi_{2 r}^{2}(\gamma / 2)\right\}=\gamma / 2$.

Portanto, um intervalo de credibilidade $100(1-\gamma) \%$ para $\theta_{1}$ condicionado aos dados é dado por:

$$
i c\left(\theta_{1}\right)=\left[\frac{2 \sum_{i=1}^{k} A_{i} V_{i}^{\beta}}{V_{1}^{\beta} \chi_{2 r}^{2}(1-\gamma / 2)} ; \frac{2 \sum_{i=1}^{k} A_{i} V_{i}^{\beta}}{V_{1}^{\beta} \chi_{2 r}^{2}(\gamma / 2)}\right]
$$




\section{Capítulo 4}

\section{Planejamento de Experimentos}

Neste capítulo será apresentado um estudo sobre planejamento de experimentos para os testes de sobrevivência acelerados. Considerando $\mathrm{k}$ níveis de stress e um conjunto de dados observados, tem-se por objetivo encontrar o número necessário de falhas para um nível adicional de stress. Através deste estudo de planejamento, também é possível determinar o número de níveis de stress que devem ser considerados.

O interesse da indústria está voltado para experimentos com custo menor. Isto é, associando-se um custo operacional a cada unidade que falha, o custo total do experimento envolve o número total de falhas. Assim, torna-se importante estimar o número necessário (mínimo) de falhas.

Estando interessado em adicionar um nível de stress $V_{k+1}$ ao seu experimento, o pesquisador fixa o comprimento do intervalo de credibilidade, por exemplo um intervalo de comprimento máximo igual à $\mathrm{L}$, associado a uma probabilidade $(1-\gamma)$. Portanto, deseja - se encontrar o número necessário de fallhas, $r_{k+1}$, usando a informação dos dados observados.

\subsection{Planejamento Seqüencial}

Considerando o parâmetro $\beta$ conhecido, o conjunto de dados observados em uma primeira etapa do experimento, é denotado por dados 1, onde o número total de itens colocados em teste é $n=n_{1}+\cdots+n_{k}$, o número total de falhas obtidas é $r=$ $r_{1}+\cdots+r_{k}$ para os $\mathrm{k}$ níveis de stress $V_{1}, \ldots, V_{k}$ fixados e as estatísticas $A_{1}, \ldots, A_{k}$, 
onde $A_{i}=\sum_{j=1}^{r_{i}} t_{j i}+\left(n_{i}-r_{i}\right) t_{r_{i} i}$ para $i=1, \ldots, k$ são valores já determinados (Capítulos 1 e 2) e por isso, conhecidos nesta etapa.

Suponha que baseado nos dados 1 , o pesquisador encontre um intervalo de credibilidade de comprimento $L_{1}$ associado à probabilidade $(1-\gamma)$, onde $L_{1}$ é maior do que um comprimento desejado $L\left(L<L_{1}\right)$.

Da análise feita a partir dos dados 1 , foi obtida a densidade a posteriori para $\alpha:$

$$
\Pi(\alpha \mid \text { dados } 1)=\frac{\left(\sum_{i=1}^{k} A_{i} V_{i}^{\beta}\right)^{r} \alpha^{-(r+1)}}{\Gamma(r)} \exp \left\{-\frac{\sum_{i=1}^{k} A_{i} V_{i}^{\beta}}{\alpha}\right\}, \quad \alpha>0
$$

e a posteriori para $\theta_{1}$ :

$$
\Pi\left(\theta_{1} \mid \text { dados } 1\right)=\frac{\left(\sum_{i=1}^{k} A_{i} V_{i}^{\beta}\right)^{r}}{\Gamma(r) V_{1}^{\beta r} \theta_{1}^{r+1}} \exp \left\{-\frac{\sum_{i=1}^{k} A_{i} V_{i}^{\beta}}{V_{1}^{\beta} \theta_{1}}\right\}, \quad \theta_{1}>0
$$

dadas respectivamente em (3.8) e (3.9).

Numa segunda etapa do experimento, assuma que os dados foram coletados num nível adicional de stress $V_{k+1} \operatorname{com} n_{k+1}$ unidades obtendo $r_{k+1}$ falhas, e a estatística:

$$
A_{k+1}=\sum_{j=1}^{r_{k+1}} t_{j,(k+1)}+\left(n_{k+1}-r_{k+1}\right) t_{r_{k+1}, k+1}
$$

Nesta segunda etapa considere como priori para $\alpha$ a densidade a posteriori para $\alpha$ baseada nos dados 1 . Ou seja, a informação obtida através de uma primeira análise passa agora a ser incorporada como densidade a priori atualizando a densidade a posteriori baseada nos dados 2 . Assim, a priori $\Pi(\alpha)$ é dada por $\Pi(\alpha \mid$ dados 1$)(\operatorname{ver}(3.8))$.

A função de verossimilhança para $\alpha$ baseada nos dados 2 é dada da mesma maneira considerando os conceitos de estatística de ordem e de função de verossimilhança. Nesta segunda etapa, tendo apenas um nível adicional $V_{k+1}, L(\alpha \mid$ dados2) é dada por:

$$
\begin{aligned}
L(\alpha \mid \text { dados } 2) & =f\left(t_{j, k+1} ; \lambda_{k+1}\right) S^{\left(n_{k+1}-r_{k+1}\right)}\left(t_{r_{k+1}, k+1} ; \lambda_{k+1}\right) \\
& =\lambda_{k+1}^{r_{k+1}} \exp \left\{-\lambda_{k+1} A_{k+1}\right\} \\
& =\left(\frac{V_{k+1}^{\beta}}{\alpha}\right)^{r_{k+1}} \exp \left\{-\frac{V_{k+1}^{\beta} A_{k+1}}{\alpha}\right\}
\end{aligned}
$$


Para a construção de um intervalo de credibilidade para $\theta_{1}$, com o nível adicional de stress $V_{k+1} \operatorname{com} r_{k+1}$ falhas, a mesma linha de raciocínio anterior é seguida, isto é, pela técnica de transformação de variáveis, verifica-se que a densidade a posteriori para $\theta_{1}$ baseada nos dados 2 onde $\theta_{1}=\alpha / V_{1}^{\beta}$ isto é, $\alpha=\theta_{1} V_{1}^{\beta}$ e $\left|d \alpha / d \theta_{1}\right|=V_{1}^{\beta}$, é dada por:

$$
\Pi\left(\theta_{1} \mid \text { dados } 2\right)=\frac{\left(\sum_{i=1}^{k} A_{i} V_{i}^{\beta}\right)^{r+r_{k+1}}}{V_{1}^{\beta\left(r+r_{k+1}\right)} \theta_{1}^{\left(r+r_{k+1}\right)} \Gamma\left(r+r_{k+1}\right)} \exp \left\{-\frac{\sum_{i=1}^{k+1} A_{i} V_{i}^{\beta}}{\theta_{1} V_{1}^{\beta}}\right\}, \quad \theta_{1}>0
$$

Portanto,

$$
X \mid \text { dados }=2 \frac{\sum_{i=1}^{k+1} A_{i} V_{i}^{\beta}}{\theta_{1} V_{1}^{\beta}} \sim \chi_{2\left(r+r_{k+1}\right)}^{2}
$$

Um intervalo de credibilidade para $\theta_{1}$ adicionando o nível de stress $V_{k+1}$, com probabilidade $(1-\gamma)$ é dado por:

$$
P\left\{\chi_{2\left(r+r_{k+1}\right)}^{2}(\gamma / 2) \leq X \leq \chi_{2\left(r+r_{k+1}\right)}^{2}(1-\gamma / 2)\right\}=1-\gamma
$$

isto é,

$$
P\left\{\chi_{2\left(r+r_{k+1}\right)}^{2}(\gamma / 2) \leq 2 \frac{\sum_{i=1}^{k+1} A_{i} V_{i}^{\beta}}{\theta_{1} V_{1}^{\beta}} \leq \chi_{2\left(r+r_{k+1}\right)}^{2}(1-\gamma / 2)\right\}=1-\gamma
$$

Portanto, um intervalo de credibilidade $100(1-\gamma) \%$ para $\theta_{1}$ baseado nos dados 1 e 2, é dado por:

$$
i c\left(\theta_{1}\right)=\left[\frac{2 \sum_{i=1}^{k+1} A_{i} V_{i}^{\beta}}{V_{1}^{\beta} \chi_{2\left(r+r_{k+1}\right)}^{2}(1-\gamma / 2)} ; \frac{2 \sum_{i=1}^{k+1} A_{i} V_{i}^{\beta}}{V_{1}^{\beta} \chi_{2\left(r+r_{k+1}\right)}^{2}(\gamma / 2)}\right]
$$

O comprimento deste intervalo é dado por:

$$
L=\frac{2\left(\sum_{i=1}^{k+1} A_{i} V_{i}^{\beta}\right)}{V_{1}^{\beta}}\left\{\frac{1}{\chi_{2\left(r+r_{k+1}\right)}^{2}(\gamma / 2)}-\frac{1}{\chi_{2\left(r+r_{k+1}\right)}^{2}(1-\gamma / 2)}\right\}
$$

\subsection{Determinação de $r_{k+1}$}

Suponha que os dados 1 são conhecidos e que o pesquisador tenha especificado um comprimento $L$ para o intervalo de credibilidade para $\theta_{1}$, associado à probabilidade 
$\left(1-\gamma^{*}\right)$. O objetivo nesta etapa é estimar o número necessário de falhas no segundo estágio do experimento.

Considerando um coeficiente de confiança $100\left(1-\gamma^{*}\right) \%$ e observando o fato que $A_{k+1}$ é uma variável aleatória, quer-se encontrar $r_{k+1}$ de (4.3) tal que,

$$
P\left\{\frac{2\left(\sum_{i=1}^{k} A_{i} V_{i}^{\beta}+A_{k+1} V_{k+1}^{\beta}\right) a\left(r_{k+1}\right)}{V_{1}^{\beta}} \leq L\right\}=1-\gamma^{*}
$$

onde $\beta$ e L são especificados anteriormente, $V_{1}, \ldots, V_{k+1}$ são níveis de stress fixados e:

$$
\left\{\begin{aligned}
r & =\sum_{i=1}^{k} r_{i} \text { é fixo } \\
A_{k+1} & =\sum_{j=1}^{r_{k+1}} t_{j, r_{k+1}}+\left(n_{k+1}-r_{k+1}\right) t_{r_{k+1}, k+1} \\
a\left(r_{k+1}\right) & =\left(\chi_{2\left(r+r_{k+1}\right)}^{2}(\gamma / 2)\right)^{-1}-\left(\chi_{2\left(r+r_{k+1}\right)}^{2}(1-\gamma / 2)\right)^{-1}
\end{aligned}\right.
$$

Escrevendo $\quad B=\sum_{i=1}^{k} A_{i} V_{i}^{\beta}$, tem-se:

$$
P\left\{\frac{2\left(B+A_{k+1} V_{k+1}^{\beta}\right) a\left(r_{k+1}\right)}{V_{1}^{\beta}} \leq L\right\}=1-\gamma^{*}
$$

Note que se

$$
\frac{2\left(B+A_{k+1} V_{k+1}^{\beta}\right) a\left(r_{k+1}\right)}{V_{1}^{\beta}} \leq L
$$

então, $B+A_{k+1} V_{k+1}^{\beta} \leq L . V_{1}^{\beta} / 2 a\left(r_{k+1}\right)$

$$
\text { Portanto, } A_{k+1} \leq\left\{\frac{L V_{1}^{\beta}}{2 a\left(r_{k+1}\right)}-B\right\} / V_{k+1}^{\beta}
$$

Resultado 4.1 Considerando a função densidade(2.1) $e \quad a$ estatística $A_{i}=\sum_{j=1}^{r_{i}} t_{i j}+\left(n_{i}-r_{i}\right) t_{r_{i} i}, i=1, \ldots, k$, então:

$$
\frac{2 A_{i}}{\theta_{i}} \sim \chi_{2 r_{i}}^{2}
$$

onde $\theta_{i}=\frac{1}{\lambda_{i}}$.

(ver por exemplo, Lawless, 1982). 
Então,

Em particular, considere aqui, o parâmetro $\theta_{k+1}$ e o nível de stress $k+1$.

onde $\theta_{k+1}=\frac{\alpha}{V_{k+1}^{\beta}} \cdot$ Ou seja,

$$
\frac{2 A_{k+1}}{\theta_{k+1}} \sim \chi_{2 \tau_{k+1}}^{2}
$$

$$
Y=\frac{2 A_{k+1} V_{k+1}^{\beta}}{\alpha} \sim \chi_{2 \tau_{k+1}}^{2}
$$

Como $A_{k+1} \leq \frac{1}{V_{k+1}^{\beta}}\left\{\frac{L V_{1}^{\beta}}{2 a\left(r_{k+1}\right)}-B\right\}$, então:

$$
Y \leq \frac{2}{\alpha}\left\{\frac{L V_{1}^{\beta}}{2 a\left(r_{k+1}\right)}-B\right\}
$$

Portanto, para determinar $r_{k+1}$, deve-se achar $r_{k+1}$, tal que:

$$
P\left\{Y \leq \frac{2}{\alpha}\left(\frac{L V_{1}^{\beta}}{2 a\left(r_{k+1}\right)}-B\right)\right\} \cong 1-\gamma^{*}
$$

onde $\left(1-\gamma^{*}\right)$ é fixado e $Y \sim \chi_{2 r_{k+1}}^{2}$ dado em (4.5).

Pelo fato do parâmetro $\alpha$ ser desconhecido, pode-se usar um estimador de $\alpha$. Por exemplo, considere a moda da densidade a posteriori para $\alpha$ baseada nos dados 1 . Como a densidade a posteriori é dada por:

$$
\Pi(\alpha \mid \text { dados } 1)=\frac{\alpha^{-(r+1)}}{\Gamma(r)}\left\{\sum_{i=1}^{k} A_{i} V_{i}^{\beta}\right\}^{r} \exp \left\{-\frac{\sum_{i=1}^{k} A_{i} V_{i}^{\beta}}{\alpha}\right\}
$$

a maximização desta equação fornece um estimador pontual de $\alpha$ dado pela moda a posteriori:

$$
\text { de }\left.\quad \frac{d \ln \Pi(\alpha \mid \text { dados } 1)}{d \alpha}\right|_{\alpha=\bar{\alpha}}=0 \quad \text { tem - se } \quad-(r+1)+\frac{B}{\alpha}=0
$$

Portanto, a moda $\tilde{\alpha}$ é dada por,

$$
\tilde{\alpha}=\frac{\sum_{i=1}^{k} A_{i} V_{i}^{\beta}}{r+1}
$$

Assim, usando (4.6) encontra-se o número necessário de falhas $r_{k+1}$ para um nivel adicional de stress $V_{k+1}$, com um intervalo de credibilidade $100\left(1-\gamma^{*}\right) \%$ para $\theta_{1}$ com comprimento $\mathrm{L}$. 


\section{Capítulo 5}

\section{Densidades Preditivas}

Neste capítulo será apresentado um estudo sobre densidades preditivas com aplicações em controle de qualidade e planejamento de experimentos em testes acelerados. Assuma que os tempos de sobrevivência dos componentes observados têm distribuição Exponencial e a relação entre o parâmetro da distribuição de vida e o stress é dada pelo Modelo de Lei de Potência Inversa.

$\mathrm{O}$ uso de densidades preditivas pode ser de grande interesse prático, principalmente na área de confiabilidade (ver por exemplo Geisser, 1982).

Considerando o teste de sobrevivência acelerado descrito no Capítulo 2, a densidade preditiva para uma observação futura em um nível $i$ de stress $V$ é determinada nesta secção. Inferências sobre observações futuras serão obtidas a partir da densidade preditiva encontrada.

Resultado 5.1 Sejam $T_{1} \ldots, T_{m}$ variáveis com função densidade conhecida, associada aos parâmetros $\theta_{1}, \ldots, \theta_{p}$ pertencente ao espaço paramétrico $\Theta$, onde $T_{j}>0$ representa $o$ tempo de sobrevivência da $j$-ésima observação, $j=1, \ldots, m$. A densidade preditiva para uma observação futura $T_{m+1}$, é dada por:

$$
f\left(t_{m+1} \mid t_{1}, \ldots, t_{m}\right)=\int_{\Theta} f\left(t_{m+1} \mid \theta_{1}, \ldots, \theta_{p}\right) \Pi\left(\theta_{1}, \ldots, \theta_{p} \mid t_{1}, \ldots, t_{m}\right) d \theta_{1} \ldots d \theta_{p}
$$

isto é, $\quad f\left(t_{m+1} \mid t_{1}, \ldots, t_{m}\right)=E\left\{f\left(t_{m+1} \mid \theta_{1} \ldots, \theta_{p}\right)\right\}$

onde $f\left(t_{m+1} \mid \theta_{1}, \ldots, \theta_{p}\right)$ é a função densidade de $T_{m+1}$ e $\Pi\left(\theta_{1}, \ldots, \theta_{p} \mid t_{1}, \ldots, t_{m}\right)$ é a densidade a posteriori dos parâmetros $\theta_{1}, \ldots, \theta_{p}$. 
(ver por exemplo, Aitchison \& Dunsmore, 1975).

Para o caso em estudo, considere o experimento descrito no Capítulo 2: $n_{i}$ itens são submetidos a um teste sob condições aceleradas de stress $V_{i}$, até a obtenção de $r_{i}$ falhas. Os tempos de sobrevivência dos itens, $t_{i j}, j=1, \ldots, r_{i}$ e $i=1, \ldots, k$ têm distribuição Exponencial (2.1) e a relação entre o parâmetro da distribuição de vida $\lambda_{i} \mathrm{e}$ o stress $V_{i}$ do Modelo de Lei de Potência Inversa (2.2) é dada por $\theta_{i}=\frac{1}{\lambda_{i}}, i=1, \ldots, k$.

Assumindo uma priori de Jeffreys para os parâmetros $\alpha$ e $\beta$ do modelo (2.2), $\Pi(\alpha, \beta) \propto \frac{1}{\alpha}, \alpha$ e $\beta \in \Theta$, onde $\Theta=\{(\alpha, \beta): \alpha>0$ e $-\infty<\beta<\infty\}$, a densidade a posteriori para $\alpha$ e $\beta$ encontrada em (3.2) é dada por:

$$
\Pi(\alpha, \beta \mid \text { dados }) \propto \alpha^{-(r+1)} \sum_{i=1}^{k} V_{i}^{\beta r_{i}} \exp \left\{-\frac{\sum_{i=1}^{k} A_{i} V_{i}^{\beta}}{\alpha}\right\}
$$

Observe que $f\left(t_{(n+1) i} \mid \alpha, \beta\right)$ é dada por:

$$
f\left(t_{(n+1) i} \mid \alpha, \beta\right)=\frac{V_{i}^{\beta}}{\alpha} \exp \left\{-\frac{V_{i}^{\beta}}{\alpha} t_{(n+1) i}\right\}
$$

Portanto, a função densidade preditiva para uma observação $T_{(n+1) i}(\operatorname{ver}(5.1))$, em um nível $i$ do stress $V$ é dada por:

$$
\begin{aligned}
f^{i}\left(t_{(n+1) i} \mid \text { dados }\right) & \propto \int_{-\infty}^{\infty} \int_{0}^{\infty} \frac{V_{i}^{\beta}}{\alpha} \exp \left\{\frac{-V_{i}^{\beta}}{\alpha} t_{(n+1) i}\right\} \alpha^{-(r+1)} \\
& \times \sum_{i=1}^{k} V_{j}^{\beta r_{j}} \exp \left\{-\frac{\sum_{j=1}^{k} A_{j} V_{j}^{\beta}}{\alpha}\right\} d \alpha d \beta \\
& \propto \int_{-\infty}^{\infty} V_{i}^{\beta} \prod_{j=1}^{k} V_{j}^{\beta r_{j}}\left[\int_{0}^{\infty} \alpha^{-(r+2)}\right. \\
& \left.\times \exp \left\{-\frac{1}{\alpha}\left(V_{i}^{\beta} t_{(n+1) i}+\sum_{j=1}^{k} A_{j} V_{j}^{\beta}\right)\right\} d \alpha\right] d \beta
\end{aligned}
$$

Pelo Resultado (3.1), tem-se:

$$
f^{i}\left(t_{(n+1) i} \mid d a d o s\right) \propto \int_{-\infty}^{\infty} \frac{V_{i}^{\beta} \prod_{j=1}^{k} V_{j}^{\beta r_{j}}}{\left\{V_{i}^{\beta} t_{(n+1) i}+\sum_{j=1}^{k} A_{j} V_{j}^{\beta}\right\}^{r+1}} d \beta
$$


aplicado:

Reescrevendo (5.2) de maneira conveniente, o Método de Laplace pode ser

$$
f^{i}\left(t_{(n+1) i} \mid t_{1}, \ldots, t_{n}\right) \propto \int_{-\infty}^{\infty} e^{-n h(\beta)} d \beta
$$

onde $n h(\beta)=-\beta \ln V_{i}-\beta \sum_{j=1}^{k} r_{j} \ln V_{j}+(r+1) \ln \left\{V_{i}^{\beta} t_{(n+1) i}+\sum_{j=1}^{k} A_{j} V_{j}^{\beta}\right\}$

Pelo Método de Laplace, a densidade preditiva para $T_{(n+1) i}$ é dada por:

$$
f^{i}\left(t_{(n+1) i} \mid t_{1}, \ldots, t_{n}\right) \propto \frac{\left\{n h^{\prime \prime}(\hat{\beta})\right\}^{-\frac{1}{2}} V_{i}^{\hat{\beta}} \prod_{j=1}^{k} V_{j}^{\hat{\beta}_{r_{j}}}}{\left\{V_{i}^{\hat{\beta}} t_{(n+1) i}+\sum_{j=1}^{k} A_{j} V_{j}^{\hat{\beta}}\right\}^{\tau+1}}, t_{(n+1) i}>0
$$

onde $\hat{\beta}$ que maximiza $-n h(\beta)$ é tal que satisfaça a igualdade:

$$
\ln V_{i}+\sum r_{j} \ln V_{j}=(r+1) \frac{\left[\left(\ln V_{i}\right) V_{i}^{\hat{\beta}} t_{(n+1) i}+\sum_{j=1}^{k} A_{j} V_{j}^{\hat{\beta}} \ln V_{j}\right]}{\left[V_{i}^{\hat{\beta}} t_{(n+1) i}+\sum_{j=1}^{k} A_{j} V_{j}^{\hat{\beta}}\right]}
$$

e $n h^{\prime \prime}(\hat{\beta})$ é dado por:

$(r+1)\left\{\frac{V_{i}^{\beta}\left(\ln V_{i}\right)^{2} t_{(n+1) i}+\sum_{j=1}^{k} A_{j} V_{j}^{\beta}\left(\ln V_{j}\right)^{2}-\left(V_{i}^{\beta}\left(\ln V_{i}\right) t_{(n+1) i}+\sum_{j=1}^{k} A_{j} V_{j}^{\beta} \ln V_{j}\right)^{2}}{\left(V_{i}^{\beta} t_{(n+1) i}+\sum_{j=1}^{k} A_{j} V_{j}^{\beta}\right)}\right\}$

Assumindo que o parâmetro $\beta$ seja conhecido, (ver Capítulo 3 , secção 3.2 ), a densidade a priori de Jeffreys para $\alpha$ é dada por $\Pi(\alpha) \propto \frac{1}{\alpha}, \alpha>0$. Assim, a densidade a posteriori para $\alpha$ é dada por,

$$
\Pi(\alpha \mid \text { dados })=\frac{\left\{\sum_{j=1}^{k} A_{j} V_{j}^{\beta}\right\}^{r}}{\Gamma(r)} \alpha^{-(r+1)} \exp \left\{\frac{-\sum_{j=1}^{k} A_{j} V_{j}^{\beta}}{\alpha}\right\}, \quad \alpha>0
$$

$(\operatorname{ver}(3.8))$. 
Portanto, por (5.1), tem-se que:

$$
\begin{aligned}
f^{i}\left(t_{(n+1) i} \mid t_{1}, \ldots, t_{n}\right) & =\int_{0}^{\infty} \frac{V_{i}^{\beta}\left\{\sum_{j=1}^{k} A_{j} V_{j}^{\beta}\right\}^{r}}{\alpha \Gamma(r)} \alpha^{-(r+1)} \\
& \times \exp \left\{-\frac{1}{\alpha}\left[V_{i}^{\beta} t_{(n+1) i}+\sum_{j=1}^{k} A_{j} V_{j}^{\beta}\right]\right\} d \alpha \\
& =\frac{V_{i}^{\beta}\left\{\sum_{j=1}^{k} A_{j} V_{j}^{\beta}\right\}^{r}}{\Gamma(r)} \int_{0}^{\infty} \alpha^{-(r+2)} \\
& \times \exp \left\{-\frac{1}{\alpha}\left[V_{i}^{\beta} t_{(n+1) i}+\sum_{j=1}^{k} A_{j} V_{j}^{\beta}\right]\right\} d \alpha
\end{aligned}
$$

Usando o Resultado (3.1) e como $\Gamma(r+1)=r \Gamma(r)$, a função densidade preditiva para uma observação futura em um nivel $i$ do stress $V, T_{(n+1) i}$ assumindo $\beta$ conhecido, é dada por:

$$
f^{i}\left(t_{(n+1) i} \mid t_{1}, \ldots, t_{n}\right)=\frac{r V_{i}^{\beta}\left\{\sum_{j=1}^{k} A_{j} V_{j}^{\beta}\right\}^{r}}{\left\{V_{i}^{\beta} t_{(n+1) i}+\sum_{j=1}^{k} A_{j} V_{j}^{\beta}\right\}^{r+1}}, \quad t_{(n+1) i}>0
$$

Isto é, $T_{(n+1) i} \mid T_{1}, \ldots, T_{n}$ tem uma distribuição de Pareto.

forma:

Escrevendo $C=\sum_{j=1}^{k} A_{j} V_{j}^{\beta}$, a densidade (5.4) pode ser reescrita da seguinte

$$
f^{i}\left(t_{(n+1) i} \mid t_{1}, \ldots, t_{n}\right)=\frac{r\left(\frac{C}{V_{i}^{\beta}}\right)^{r}}{\left\{t_{(n+1) i}+\frac{C}{V_{i}^{\beta}}\right\}^{r+1}}, \quad t_{(n+1) i}>0
$$

Usando a técnica de transformação de variáveis, ou seja, definindo, $Y_{n+1}=b+T_{n+1}, a=r$ e $b=C / V_{i}^{\beta}$, tem-se:

$$
f\left(y_{(n+1)} \mid t_{1}, \ldots, t_{n}\right)=\frac{a b^{a}}{y_{n+1}^{a+1}}, \quad y>b
$$

Observe em (5.6), que a função densidade preditiva para $Y_{n+1}$ tem, para $a>1$,

$$
E\left(Y_{n+1} \mid t_{1}, \ldots, t_{n}\right)=\frac{a b}{a-1}
$$


e para $a>2$

$$
\operatorname{var}\left(Y_{n+1} \mid t_{1}, \ldots, t_{n}\right)=\frac{a b^{2}}{(a-1)^{2}(a-2)}
$$

Assim, considerando o tempo de sobrevivência $t_{(n+1) i}$ em um nivel de stress $V_{i}$, tem-se que:

$$
\begin{gathered}
E^{i}\left(T_{(n+1) i} \mid t_{1}, \ldots, t_{n}\right)=\frac{\sum_{j=1}^{k} A_{j} V_{j}^{\beta}}{(r-1) V_{i}^{\beta}} \\
\operatorname{Var}^{i}\left(T_{(n+1) i} \mid t_{1}, \ldots, t_{n}\right)=\frac{r\left(\sum_{j=1}^{k} A_{j} V_{j}^{\beta}\right)^{2}}{(r-1)^{2}(r-2) V_{i}^{2 \beta}},
\end{gathered}
$$

para $i=1, \ldots, k$.

\subsection{Densidades Preditivas em Controle de Qualida- de}

Uma aplicação de densidades preditivas pode ser dada em Engenharia, na área de controle de qualidade.

$\mathrm{O}$ pesquisador interessado em adquirir componentes fabricados por uma indústria, deseja saber se o componente satisfaz ou não as especificações do projeto. Para verificar se um processo está sob controle, o engenheiro retira uma amostra, de cada lote, dos componentes fabricados e verifica se as peças atendem às especificações do pesquisador.

Usualmente, para minimizar o custo e o tempo do experimento, testes com níveis de stress acelerados são aplicados por um período de tempo $P_{i}$.

Assumindo $\beta$ conhecido, considere a densidade preditiva dada em (5.5). Fixando uma probabilidade $(1-\gamma)$ e um nível i de stress $\mathrm{V}$, um valor de $P_{i}$ pode ser encontrado, satisfazendo:

$$
P^{i}\left\{T_{(n+1) i}>P_{i} \mid \text { dados }\right\}=1-\gamma
$$

Da mesma forma, fixando-se o período de tempo $P_{i}$ de aplicação do stress, o valor de $V_{i}$ pode ser encontrado, tal que satisfaça a equação (5.9), isto é, 


$$
P^{i}\left\{T_{(n+1) i}>P_{i} \mid \text { dados }\right\}=\int_{P_{i}}^{\infty} f^{i}\left(t_{n+1) i} \mid d a d o s\right) d t_{(n+1) i}
$$

Daí,

$$
P^{i}\left\{T_{(n+1) i}>P_{i} \mid \text { dados }\right\}=\int_{P_{i}}^{\infty} \frac{V_{i}^{\beta}\left[\sum_{j=1}^{k} A_{j} V_{j}^{\beta}\right]^{r}}{\left\{V_{i}^{\beta} t_{(n+1) i}+\sum_{j=1}^{k} A_{j} V_{j}^{\beta}\right\}^{r+1}} d t_{(n+1) i}
$$

ou ainda, substituindo a forma simplificada encontrada em (5.6), tem-se que,

$$
P^{i}\left\{T_{(n+1) i}>P_{i} \mid \text { dados }\right\}=r\left(\frac{C}{V_{i}^{\beta}}\right)^{r} \int_{P_{i}}^{\infty} \frac{d t_{(n+1) i}}{\left\{t_{(n+1) i}+\frac{C}{V_{i}^{\beta}}\right\}^{r+1}}
$$

onde $C=\sum_{i=1}^{k} A_{i} V_{i}^{\beta}$.

Considerando $P^{i}\left\{T_{(n+1) i}>P_{i} \mid\right.$ dados $\}=1-\gamma$, o resultado da integração acima é dado por:

$$
\left(\frac{C}{V_{i}^{\beta}}\right)^{r}\left(\frac{1}{P_{i}+\frac{C}{V_{i}^{\beta}}}\right)^{r}=1-\gamma
$$

A partir de (5.10), é possível então determinar os valores de $P_{i}$ ou $V_{i}$, assumindo $\beta$ conhecido, que satisfaçam (5.6) ou (5.9) respectivamente. Assim,

(i) Fixando-se um nível $i$ de stress $V$, o período de tempo $P_{i}$ a ser aplicado no stress $V_{i}$ é dado por:

$$
P_{i}=\frac{C\left[1-(1-\gamma)^{\frac{1}{r}}\right]}{(1-\gamma)^{\frac{1}{r}} V_{i}^{\beta}}
$$

(ii) Fixando-se o período $P_{i}$ de aplicação do stress, o nivel $V_{i}$ é dado por:

$$
V_{i}=\left\{\frac{C\left(1-(1-1 / \gamma)^{\frac{1}{r}}\right)}{(1-\gamma)^{\frac{1}{r}} P_{i}}\right\}^{1 / \beta}
$$


Para os valores $P_{i}$ e $V_{i}$ encontrados satisfazendo $P^{i}\left\{T_{(n+1) i}>P_{i} \mid\right.$ dados $\}=$ $1-\gamma$, é possível estabelecer um critério para o teste de controle de qualidade.

Observar m componentes submetidos a um teste de sobrevivência sob o nível de stress $V_{i}$ durante um período de tempo $P_{i}$.

Definindo:

$$
\begin{aligned}
\gamma^{i}=P^{i}\left\{T_{(n+1) i} \leq P_{i} \mid \text { dados }\right\}, \text { considere o teste de hipótese: } \\
\\
\left\{\begin{array}{c}
H_{0}: \gamma^{i} \leq \gamma \text { (o processo está sob controle) } \\
H_{1}: \gamma^{i}>\gamma \text { (o processo está fora de controle) }
\end{array}\right.
\end{aligned}
$$

Seja $X$ o número de componentes que falharam $(X \leq m)$ durante o teste. Assumindo $X \sim b\left(m ; \gamma^{i}\right)$, tem-se:

$$
X \stackrel{a}{\sim} N\left\{m \gamma^{i} ; m \gamma^{i}\left(1-\gamma^{i}\right)\right\}
$$

ou seja, pelo Teorema do Limite Central, quando n cresce, a distribuição Binomial tende à curva normal. Em geral, pode - se considerar que se $m \gamma \geq 5$ e $m(1-\gamma) \geq 5$, então m já é suficientemente grande para se utilizar a aproximação da distribuição Binomial para a distribuição Normal. Alguns autores porém, sugerem $m \gamma \geq 10$ e $m(1-\gamma) \geq 10$ ou ainda $m \gamma \geq 15$ e $m(1-\gamma) \geq 15$ (ver por exemplo, Bickel \& Doksum, 1977).

Então, um critério para o teste de controle de qualidade é dado por:

o processo está fora de controle se $Z \geq Z_{\alpha}$, onde

$$
Z=\frac{(X-m \gamma)}{\sqrt{m \gamma(1-\gamma)}} \stackrel{a}{\sim} N\{0,1\}
$$

$\alpha$ é o nível de significância e $Z_{\alpha}$ é tal que $P\left[Z \geq Z_{\alpha}\right]=\alpha$.

Assumindo $\beta$ desconhecido, a densidade preditiva aproximada pelo Método de Laplace, dada em (5.3) e métodos numéricos podem ser usados. Conseqüentemente, os valores $V_{i}$ e $P_{i}$ podem ser encontrados satisfazendo $P^{i}\left\{T_{(n+1) i}>P_{i} \mid\right.$ dados $\}=(1-\gamma)$. 


\section{Capítulo 6}

\section{Análise Bayesiana assumindo uma Distribuição de Weibull para os tempos de sobrevivência}

A distribuição de Weibull tem grande aplicabilidade em modelos de sobrevivência, pois representa um modelo apropriado para descrever a confiabilidade e o sistema de falha de componentes, quando estes apresentam variações em suas taxas de falha.

Em problemas práticos, é possível verificar se os tempos de vida dos componentes apresentam variações na taxa de falha, ou se esta se mantém constante no decorrer do tempo de uso. Ou seja, é possível verificar se a distribuição do tempo de falha dos itens pode ser ajustada à distribuição de Weibull, levando-se em consideração uma forte dependência do risco de falha sobre o fator tempo, ou se pode ser ajustada à distribuição Exponencial, onde apresentam constância na taxa de falha ou ainda, pequenas variações que podem ser consideradas constantes.

Um método alternativo às análises gráficas (ver por exemplo Nelson,1972), é apresentado neste capítulo para verificar a melhor distribuição ajustada para os tempos de sobrevivência dos componentes.

Considere um teste de sobrevivência acelerado em um planejamento com censuras do Tipo II, onde são aplicados nos itens, $\mathrm{k}$ valores de stress $V_{i}, i=1,2, \ldots, k$, como descrito no Capítulo 2. O comportamento de falha desses componentes é especificado pela distribuição de Weibull. 
Seja então, $f\left(t ; \lambda_{i}^{*}, p\right)$, a função densidade de probabilidade da variável aleatória tempo de falha, $T>0$, para um nivel i de stress $\mathrm{V}$, dada por:

$$
f\left(t ; \lambda_{i}^{*}, p\right)= \begin{cases}\lambda_{i}^{*} p\left(\lambda_{i}{ }^{*} t\right)^{p-1} \exp \left\{-\left(\lambda_{i}^{*} t\right)^{p}\right\} & \lambda_{i}^{*}>0, t>0 \text { e } p>0 \\ 0 & \text { caso contrário }\end{cases}
$$

onde $\theta_{i}^{*}=\frac{\Gamma(1+1 / p)}{\lambda_{i}^{*}}$ é o tempo médio de sobrevivência dos componentes para $i=1,2, \ldots, k$ e p, chamado parâmetro de forma, é comum a todos os níveis.

Considerando o Modelo de Lei de Potência Inversa (ver Introdução), a relação existente entre o parâmetro da distribuição do tempo de sobrevivência dos componentes em estudo e a variável stress desse modelo, é dada por:

$$
\frac{1}{\lambda_{i}^{*}}=\frac{\alpha}{V_{i}^{\beta}}
$$

onde $\alpha$ e $\beta \in \Theta$ e $i=1,2, \ldots, k$.

A função densidade (6.1) especifica a função distribuição $F(t)$,

$$
F(t)=P[T \leq t]=1-\exp \left\{-\left(\lambda_{i}^{*} t\right)^{p}\right\}
$$

e a função de Confiabilidade,

$$
S(t)=1-F(t)=\exp \left\{-\left(\lambda_{i}^{*} t\right)^{p}\right\}
$$

como definidas no Capítulo 2.

Pela definição de função Risco, $h(t)$, verifica-se que a função densidade de Weibull tem taxa de falha dada por:

$$
h(t)=\frac{f(t)}{S(t)}=\lambda_{i}^{*} p\left(\lambda_{i}^{*} t\right)^{p-1}
$$

Note que a distribuição Exponencial é um caso particular da distribuição de Weibull, quando o parâmetro p é igual a 1. Então, testar o ajuste da distribuição Exponencial aos dados, é equivalente a estimar o parâmetro p na distribuição de Weibull e verificar se está muito próximo de 1 , isto é, $p \cong 1$.

A função de verossimilhança para os parâmetros $\lambda_{i}^{*}$ e p, com dados censurados pelo esquema de censura do Tipo II e sob um nível i do stress $\mathrm{V}$, é dada por:

$$
L_{i}\left(\lambda_{i}^{*}, p\right)=\prod_{j=1}^{r_{i}} f\left(t_{i j} ; \lambda_{i}^{*}, p\right) S^{\left(n_{i}-r_{i}\right)}\left(t_{i r_{i}} ; \lambda_{i}^{*}, p\right)
$$




$$
\begin{aligned}
& =\prod_{j=1}^{r_{i}}\left(\lambda_{i}^{*} p\left(\lambda_{i}{ }^{*} t_{i j}\right)^{p-1}\right) \exp \left\{-\left(\lambda_{i}^{*} t_{i j}\right)^{p}\right\}\left(\exp \left\{-\left(\lambda_{i}^{*} t_{r_{i}}\right)^{p}\right\}\right)^{n_{i}-r_{i}} \\
& =\left(\lambda_{i}^{*}\right)^{p r_{i}} p^{r_{i}}\left(\prod_{j=1}^{r_{i}} t_{i j}^{p-1}\right) \exp \left\{-\left(\lambda_{i}^{*}\right)^{p} \sum_{j=1}^{r_{i}} t_{i j}^{p}\right\}\left(\exp \left\{-\left(\lambda_{i}^{*}\right)^{p} t_{i r_{i}}^{p}\right\}\right)^{n_{i}-r_{i}} \\
& =\left(\lambda_{i}^{*}\right)^{p r_{i}} p^{r_{i}}\left(\prod_{j=1}^{r_{i}} t_{i j}^{p-1}\right) \exp \left\{-\left(\lambda_{i}^{*}\right)^{p} \sum_{j=1}^{r_{i}} t_{i j}^{p-1}\right\} \\
& \times \exp \left\{-\left(\lambda_{i}^{*}\right)^{p}\left(\sum_{j=1}^{r_{i}} t_{i j}^{p}+\left(n_{i}-r_{i}\right) t_{i r_{i}}^{p}\right)\right\}
\end{aligned}
$$

Definindo $A_{i}(p)=\sum_{j=1}^{r_{i}} t_{i j}^{p}+\left(n_{i}-r_{i}\right) t_{i r_{i}}^{p}$, tem-se que,

$$
L\left(\lambda_{i}^{*}, p\right)=\left(\lambda_{i}^{*}\right)^{p r_{i}} p^{r_{i}}\left(\prod_{j=1}^{r_{i}} t_{i j}^{p-1}\right) \exp \left\{-\left(\lambda_{i}^{*}\right)^{p} A_{i}(p)\right\}
$$

Assumindo $\mathrm{k}$ níveis aleatorizados de stress $V_{1}, V_{2}, \ldots, V_{k}$, a função de verossimilhança para $\lambda_{i}{ }^{*} \mathrm{e}$ p, é dada por:

$$
L\left(\lambda_{i}^{*}, p\right)=\prod_{i=1}^{k}\left[\left(\lambda_{i}^{*}\right)^{p r_{i}} p^{r_{i}}\left(\prod_{j=1}^{r_{i}} t_{i j}^{p-1}\right) \exp \left\{-\left(\lambda_{i}^{*}\right)^{p} A_{i}(p)\right\}\right]
$$

Considerando a relação (6.2),

$$
\begin{aligned}
L(\alpha, \beta, p) & =\prod_{i=1}^{k}\left[\left(\frac{V_{i}^{\beta}}{\alpha}\right)^{p r_{i}} p^{r_{i}}\left(\prod_{j=1}^{r_{i}} t_{i j}^{p-1}\right) \exp \left\{-\left(\frac{V_{i}^{\beta}}{\alpha}\right)^{p} A_{i}(p)\right\}\right] \\
& =\prod_{i=1}^{k}\left(\frac{V_{i}^{\beta}}{\alpha}\right)^{p r_{i}} p^{\sum_{i=1}^{k} r_{i}}\left\{\prod_{i=1}^{k} \prod_{j=1}^{r_{i}} t_{i j}^{p-1}\right\} \exp \left\{-\sum_{i=1}^{k}\left(\frac{V_{i}^{\beta}}{\alpha}\right)^{p} A_{i}(p)\right\} \\
& =\frac{p^{r}}{\alpha^{p r}}\left(\prod_{i=1}^{k} V_{i}^{\beta p r_{i}}\right)\left(\prod_{i=1}^{k} \prod_{j=1}^{r_{i}} t_{i j}^{p-1}\right) \exp \left\{-\frac{\sum_{i=1}^{k} V_{i}^{\beta p} A_{i}(p)}{\alpha^{p}}\right\}
\end{aligned}
$$

onde $r=\sum_{i=1}^{k} r_{i}$ é o número total de falhas para $i=1,2, \ldots, k$. 


\subsection{Análise Bayesiana assumindo $\alpha, \beta$ e p desconhe- cidos}

Nesta secção assuma que o pesquisador não tem conhecimento à priori a respeito da distribuição dos parâmetros $\alpha, \beta$ e p. Assumindo $\alpha$ e $\beta$ parâmetros independentes de p, a Regra Multiparamétrica de Jeffreys (ver por exemplo, Box \& Tiao, 1973), determina uma priori não - informativa para os parâmetros $\alpha \mathrm{e} \beta$, e considere uma priori localmente uniforme para $\mathrm{p}$.

Assim, a densidade a priori pode ser escrita como segue:

$$
\Pi(\alpha, \beta, p)=\Pi(\alpha, \beta \mid p) \Pi_{0}(p)
$$

ou ainda,

$$
\Pi(\alpha, \beta, p) \propto\{\operatorname{det} I(\alpha, \beta \mid p)\}^{1 / 2} \Pi_{0}(p)
$$

onde $I(\alpha, \beta \mid p)$ é a Matriz de Informação de Fisher dado $\mathrm{p}$, dada por:

$$
I(\alpha, \beta \mid p)=\left(\begin{array}{ll}
E\left\{-\frac{\partial^{2} l}{\partial \alpha^{2}}\right\} & E\left\{-\frac{\partial^{2} l}{\partial \alpha \partial \beta}\right\} \\
E\left\{-\frac{\partial^{2} l}{\partial \beta \partial \alpha}\right\} & E\left\{-\frac{\partial^{2} l}{\partial \beta^{2}}\right\}
\end{array}\right)
$$

onde 1 é o logaritmo da função de verossimilhança (6.3), isto é, $l(\alpha, \beta, p)=\ln L(\alpha, \beta, p)$, dado por:

$$
l(\alpha, \beta, p)=r \ln p-r p \ln \alpha+\beta p \sum_{i=1}^{k} r_{i} \ln V_{i}+(p-1) \sum_{i=1}^{k} \sum_{j=1}^{r_{i}} \ln t_{i j}-\frac{\sum_{i=1}^{k} V_{i}^{\beta p} A_{i}(p)}{\alpha^{p}}
$$

Sem assumir qualquer tipo de relação com a variável stress, Cohen (1965) encontra estimadores de máxima verossimilhança para os parâmetros de interesse, onde os dados seguem distribuição de Weibull com dois parâmetros, considerando vários esquemas amostrais.

Note que se $p=1$, a distribuição Exponencial é obtida e a expressão (6.5) é equivalente à expressão (2.6) encontrada no Capítulo 2.

Resultado 6.1 Seja T uma variável aleatória com distribuição de Weibull, isto é,

$$
T \sim W e i\left(t ; \lambda^{*}, p\right)
$$


onde $p$ é o parâmetro de forma, $\lambda^{*}>0, p>0$ e $t \geq 0$. Então,

$$
T^{p} \sim \operatorname{Exp}\{\lambda\}
$$

ou seja, $T^{p}$ tem distribuição Exponencial com parâmetro $\lambda=\left(\lambda^{*}\right)^{p}$

(ver por exemplo, Lawless, 1982).

Observando que:

$$
E\left\{A_{i}(p)\right\}=E\left\{\sum_{j=1}^{r_{i}} t_{i j}^{p}+\left(n_{i}-r_{i}\right) t_{r_{i} i}^{p}\right\}=r_{i} \alpha^{p} / V_{i}^{\beta p},
$$

as esperanças das derivadas segundas de $l(\alpha, \beta, p)$ em relação à $\alpha, \beta$ e p, são dadas por:

$$
\begin{gathered}
E\left\{-\frac{\partial^{2} l}{\partial \alpha^{2}}\right\}=\frac{r p^{2}}{\alpha^{2}} \\
E\left\{-\frac{\partial^{2} l}{\partial \alpha \partial \beta}\right\}=E\left\{-\frac{\partial^{2} l}{\partial \beta \partial \alpha}\right\}=-\frac{p^{2} \sum_{i=1}^{k} r_{i} \ln V_{i}}{\alpha} \\
E\left\{-\frac{\partial^{2} l}{\partial \beta^{2}}\right\}=p^{2} \sum_{i=1}^{k} r_{i}\left(\ln V_{i}\right)^{2}
\end{gathered}
$$

Portanto, a Matriz de informação de Fisher é dada por:

$$
I(\alpha, \beta \mid p)=\left(\begin{array}{lr}
r p^{2} / \alpha^{2} & -\left(p^{2} \sum_{i=1}^{k} r_{i} \ln V_{i}\right) / \alpha \\
-\left(p^{2} \sum_{i=1}^{k} r_{i} \ln V_{i}\right) / \alpha & p^{2} \sum_{i=1}^{k} r_{i}\left(\ln V_{i}\right)^{2}
\end{array}\right)
$$

No cálculo do determinante de $I(\alpha, \beta \mid p)$, isto é, $\operatorname{det} I(\alpha, \beta \mid p)$, note que a expressão $r \sum_{i=1}^{k} r_{i}\left(\ln V_{i}\right)^{2}-\left(\sum_{i=1}^{k} r_{i} \ln V_{i}\right)^{2}$ é constante em relação aos parâmetros. Assim,

$$
\operatorname{det} I(\alpha, \beta \mid p) \propto \frac{p^{4}}{\alpha^{2}}
$$

Considere também, uma Priori de Jeffreys localmente uniforme para $\mathrm{p}$, dada por:

$$
\Pi_{0}(p) \propto \frac{1}{p}, \quad p>0
$$


Portanto, uma priori não - informativa para $\alpha, \beta$ e p, usando (6.4), é dada por:

$$
\Pi(\alpha, \beta, p) \propto \frac{1}{p} \sqrt{\frac{p^{4}}{\alpha^{2}}}
$$

ou seja,

$$
\Pi(\alpha, \beta, p) \propto \frac{p}{\alpha}, \quad \alpha \text { e } \beta \in \Theta \text { e } p>0
$$

Determinada então $\Pi(\alpha, \beta, p)$, a densidade a posteriori conjunta para esses parâmetros é dada por:

$$
\Pi(\alpha, \beta, p \mid d a d o s)=\frac{\Pi(\alpha, \beta, p) L(\alpha, \beta, p)}{\int_{-\infty}^{\infty} \int_{0}^{\infty} \int_{0}^{\infty} \Pi(\alpha, \beta, p) L(\alpha, \beta, p) d \alpha d p d \beta}
$$

ou ainda,

$$
\Pi(\alpha, \beta, p \mid \text { dados }) \propto \Pi(\alpha, \beta, p) L(\alpha, \beta, p)
$$

Isto é,

$$
\begin{aligned}
\Pi(\alpha, \beta, p \mid d a d o s) & \propto \frac{p}{\alpha}\left(\frac{p^{r}}{\alpha^{p r}}\right)\left(\prod_{i=1}^{k} V_{i}^{\beta p r_{i}}\right)\left(\prod_{i=1}^{k} \prod_{j=1}^{r_{i}} t_{i j}^{p-1}\right) \exp \left\{-\frac{\sum_{i=1}^{k} V_{i}^{\beta p} A_{i}(p)}{\alpha^{p}}\right\} \\
& \propto \frac{p^{r+1}}{\alpha^{p r+1}}\left(\prod_{i=1}^{k} V_{i}^{\beta p r_{i}}\right)\left(\prod_{i=1}^{k} \prod_{j=1}^{r_{i}} t_{i j}^{p-1}\right) \exp \left\{-\frac{\sum_{i=1}^{k} V_{i}^{\beta p} A_{i}(p)}{\alpha^{p}}\right\}
\end{aligned}
$$

onde $\alpha$ e $\beta \in \Theta$ e $p>0$. Assim, a densidade a posteriori marginal para p é dada por:

$$
\begin{aligned}
\Pi(p \mid \text { dados }) & \propto \int_{0}^{\infty} \int_{-\infty}^{\infty} \Pi(\alpha, \beta, p \mid d a d o s) d \beta d \alpha \\
& \propto p^{r+1}\left(\prod_{i=1}^{k} \prod_{j=1}^{r_{i}} t_{i j}^{p-1}\right) \int_{0}^{\infty} \int_{-\infty}^{\infty} \prod_{i=1}^{k} V_{i}^{\beta r_{i}} \exp \left\{-\frac{V_{i}^{\beta p} A_{i}(p)}{\alpha^{p}}\right\} d \beta d \alpha(6.8)
\end{aligned}
$$

Usando o Método de Laplace para aproximações de integrais, apresentado no Apêndice $\mathrm{B}$, a expressão $\Pi(p \mid$ dados $)$ dada acima, pode ser reescrita da seguinte forma:

$$
\Pi(p \mid \text { dados }) \propto p^{r+1}\left(\prod_{i=1}^{k} \prod_{j=1}^{r_{i}} t_{i j}^{p-1}\right) \int_{-\infty}^{\infty} \int_{0}^{\infty} \exp \{-n h(\alpha, \beta)\} d \alpha d \beta
$$


onde $\quad-n h(\alpha, \beta)=-(p r+1) \ln \alpha+\beta p \sum_{i=1}^{k} r_{i} \ln V_{i}-\sum_{i=1}^{k} V_{i}^{\beta p} A_{i}(p) / \alpha^{p}$.

Então,

$$
\int \exp \{-n h(\alpha, \beta)\} d \beta d \alpha \approx \sqrt{2 \pi}\left\{\operatorname{det}\left(n D^{2} h(\hat{\alpha}, \widehat{\beta})\right)\right\}^{-1 / 2} \exp \{-n h(\hat{\alpha}, \widehat{\beta})\}
$$

onde

$$
\left\{\begin{array}{l}
(\widehat{\alpha}, \widehat{\beta}) \text { maximiza }-n h(\alpha, \beta) \\
D^{2} h(\widehat{\alpha}, \widehat{\beta}) \text { é a Matriz Hessiana de h calculada no ponto }(\widehat{\alpha}, \widehat{\beta})
\end{array}\right.
$$

ções:

Os valores $(\hat{\alpha}, \widehat{\beta})$ são encontrados resolvendo-se o seguinte sistema de equa-

$$
\left\{\begin{array} { l } 
{ \frac { \partial ( - n h ( \alpha , \beta ) ) } { \partial \alpha } = 0 } \\
{ \frac { \partial ( - n h ( \alpha , \beta ) ) } { \partial \beta } = 0 }
\end{array} \quad \text { isto é, } \quad \left\{\begin{array}{l}
-(p r+1) / \alpha+p \sum_{i=1}^{k} V_{i}^{\beta p} A_{i}(p) / \alpha^{p+1}=0 \\
p \sum_{i=1}^{k} r_{i} \ln V_{i}-\sum_{i=1}^{k} V_{i}^{\beta p} A_{i}(p) \ln V_{i}=0
\end{array}\right.\right.
$$

ou seja,

$$
\left\{\begin{array}{l}
\hat{\alpha}=\left(p \sum_{i=1}^{k} V_{i}^{\widehat{\beta} p} A_{i}(p) /(p r+1)\right)^{1 / p} \\
p / \sum_{i=1}^{k} r_{i} \ln V_{i}=(p r+1) \sum_{i=1}^{k} V_{i}^{\widehat{\beta_{p}}} A_{i}(p) / \sum_{i=1}^{k} V_{i}^{\widehat{\hat{\beta} p}} A_{i}(p)
\end{array}\right.
$$
cada p fixado.

Através de métodos iterativos, é possivel encontrar os valores $(\widehat{\alpha}, \widehat{\beta})$ para

Portanto, a densidade a posteriori marginal para $\mathrm{p}$, aproximada pelo Método de Laplace, é dada por:

$$
\begin{aligned}
\Pi(p \mid \text { dados }) & \propto p^{r+1}\left(\prod_{i=1}^{k} \prod_{j=1}^{r_{i}} t_{i j}^{p-1}\right)\left\{\operatorname{det}\left(n D^{2} h(\hat{\alpha}, \widehat{\beta})\right)\right\}^{-1 / 2} \exp \{-n h(\hat{\alpha}, \widehat{\beta})\} \\
\propto & \frac{(p r+1)^{r-1}\left(\prod_{i=1}^{k} V_{i}^{\widehat{\beta} r_{i}}\right)\left(\prod_{i=1}^{k} \prod_{j=1}^{r_{i}} t_{i j}^{p-1}\right) e^{-(p r+1) / p}}{\left(\sum_{i=1}^{k} V_{i}^{\widehat{\beta} p} A_{i}(p)\right)^{r} B(p, \widehat{\beta})}
\end{aligned}
$$

onde $p>0 \mathrm{e}$

$$
B(p, \widehat{\beta})=\left\{\frac{\sum_{i=1}^{k} V_{i}^{\widehat{\beta} p} A_{i}(p)\left(\ln V_{i}\right)^{2}}{\sum_{i=1}^{k} V_{i}^{\widehat{\beta} p} A_{i}(p)}-\left(\frac{\sum_{i=1}^{k} V_{i}^{\widehat{\beta} p} A_{i}(p) \ln V_{i}}{\sum_{i=1}^{k} V_{i}^{\widehat{\beta} p} A_{i}(p)}\right)^{2}\right.
$$


Através da maximização de (6.10), torna-se possível encontrar um estimador pontual para $\mathrm{p}$, por exemplo, a moda a posteriori de $p, \tilde{p}$.

Note que, de maneira análoga à densidade a posteriori marginal para $\mathrm{p}$, o Método de Laplace pode ser usado para encontrar a densidade a posteriori marginal para $\alpha$ ou $\beta$.

Assumindo a partir dessa análise, o parâmetro $\mathrm{p}$ conhecido, estimado através da moda da densidade a posteriori e usando o Resultado (6.1), $T^{\tilde{p}}$ tem distribuição Exponencial. Portanto, para os dados transformados, os resultados das análises anteriores, dados nos Capítulos 2 e 3, podem ser considerados.

\subsection{Posteriori Conjunta Marginal para $\theta_{1}^{*}$ e p: $\Pi\left(\theta_{1}^{*}, p \mid\right.$ dados $)$}

Nesta seç̧ão, assumindo o parâmetro p desconhecido, ou seja, considerando os tempos de sobrevivência originais, é possivel encontrar a densidade a posteriori para $o$ parâmetro de interesse $\theta_{1}^{*}$ e p conjuntamente, a partir da densidade a posteriori conjunta para $\alpha, \beta$ e p. A partir da densidade a posteriori conjunta para os parâmetros $\alpha, \beta$ e $\mathrm{p}, \Pi(\alpha, \beta, p \mid$ dados $)$ ver (6.7), considerando que os dados têm distribuição de Weibull $\mathrm{e}$ o parâmetro $\lambda_{i}^{*}$ desta distribuição está relacionado com o Modelo de Lei de Potência Inversa, $1 / \lambda_{i}^{*}=\alpha / V_{i}^{\beta}$, pode-se encontrar a densidade a posteriori conjunta marginal para $\theta_{\mathrm{i}}^{*} \mathrm{e} \mathrm{p}$, onde $\theta_{i}^{*}$ é o tempo médio de sobrevivência dos itens com distribuição de Weibull em um nível i de stress V, dado por:

$$
\theta_{i}^{*}=\frac{\Gamma(1+1 / p)}{\lambda_{i}{ }^{*}},
$$

para $i=1,2, \ldots, k$.

A densidade a posteriori conjunta para $\alpha, \beta$ e p é dada por:

$$
\begin{aligned}
\Pi(\alpha, \beta, p \mid \text { dados }) & \propto \frac{p^{n+1}}{\alpha^{p n+1}}\left[\prod_{i=1}^{k} V_{i}^{\beta p r_{i}}\right]\left[\prod_{i=1}^{k} \prod_{j=1}^{r_{i}} t_{i j}^{p-1}\right] \\
& \times \exp \left\{-\frac{\sum_{i=1}^{k} A_{i}(p) V_{i}^{\beta p}}{\alpha^{p}}\right\}
\end{aligned}
$$


onde $\left\{\begin{array}{l}\alpha \text { e } \beta \in \Theta \text { e } p>0 \\ A_{i}(p)=\sum_{j=1}^{r_{i}} t_{i j}^{p}+\left(n_{i}-r_{i}\right) t_{r_{i} i}^{p} \\ r=\sum_{i=1}^{k} r_{i} \text { é o número total de observações para } \mathrm{k} \text { amostras e } i=1,2, \ldots, k .\end{array}\right.$

O tempo médio de sobrevivência dos componentes com distribuição de Weibull, num nível de stress $\mathrm{i}$, é dado por $\theta_{i}^{*}=\Gamma(1+1 / p) / \lambda_{i}{ }^{*}$ e portanto, o tempo médio de sobrevivência dos itens nas condições normais de funcionamento é dado por,

$$
\theta_{1}^{*}=\frac{\Gamma(1+1 / p)}{\lambda_{1}^{*}} \quad \text { onde } \quad \frac{1}{\lambda_{1}^{*}}=\frac{\alpha}{V_{1}^{\beta}}
$$

Usando a técnica de transformação de variáveis, tem-se:

$$
\left\{\begin{array}{l}
\alpha=\theta_{1}^{*} V_{1}^{\beta} / \Gamma(1+1 / p) \\
\beta=\beta \\
p=p
\end{array}\right.
$$

e a matriz Jacobiana é dada por:

$$
J=\left(\begin{array}{ccc}
\partial \alpha / \partial \theta_{1}^{*} & \partial \alpha / \partial \beta & \partial \alpha / \partial p \\
\partial \beta / \partial \theta_{1}^{*} & \partial \beta / \partial \beta & \partial \beta / \partial p \\
\partial p / \partial \theta_{1}^{*} & \partial p / \partial \beta & \partial p / \partial p
\end{array}\right)
$$

ou seja,

$$
\begin{aligned}
J & =\left(\begin{array}{ccc}
\frac{V_{1}^{\beta}}{\Gamma(1+1 / p)} & \frac{\theta_{1}^{*} V_{1}^{\beta} \ln V_{1}}{\Gamma(1+1 / p)} & * \\
0 & 1 & 0 \\
0 & 0 & 1
\end{array}\right) \\
\text { Portanto, } \operatorname{det}(J) & =\frac{V_{1}^{\beta}}{\Gamma(1+1 / p)} .
\end{aligned}
$$

A partir da transformação de variáveis, a densidade a posteriori conjunta para $\theta_{1}^{*}, \beta$ e p é dada por: 


$$
\begin{aligned}
\Pi\left(\theta_{1}^{*}, \beta, p\right) & \propto \frac{p^{p r+1}}{\left(\frac{\theta_{i}^{*} V_{1}^{\beta}}{\Gamma(1+1 / p)}\right)^{p r+1}}\left(\prod_{i=1}^{k} V_{i}^{\beta p r_{i}}\right)\left(\prod_{i=1}^{k} \prod_{j=1}^{r_{i}} t_{i j}^{p-1}\right) \\
& \times \frac{V_{1}^{\beta}}{\Gamma(1+1 / p)} \exp \left\{-\frac{\sum_{i=1}^{k} A_{i}(p) V_{i}^{\beta p}}{\left(\frac{\theta_{1}^{*} V_{1}^{\beta}}{\Gamma(1+1 / p)}\right)^{p}}\right\} \\
& \propto \frac{p^{r+1}}{\left(\theta_{1}^{*}\right)^{p r+1}}\left(\frac{\Gamma(1+1 / p)}{V_{1}^{\beta}}\right)^{p r}\left(\prod_{i=1}^{k} V_{i}^{\beta p r_{i}}\right)\left(\prod_{i=1}^{k} \prod_{j=1}^{r_{i}} t_{i j}^{p-1}\right) \\
& \times \exp \left\{-\left(\frac{\Gamma(1+1 / p)}{\theta_{1}^{*}}\right)^{p} \sum_{i=1}^{k} A_{i}(p)\left(\frac{V_{i}}{V_{1}}\right)^{\beta p}\right\}
\end{aligned}
$$

onde $\theta_{1}^{*}>0,-\infty<\beta<\infty$ e $p>0$.

A densidade a posteriori marginal conjunta para $\theta_{1}^{*}$ e $\mathrm{p}$, é dada por:

$$
\begin{aligned}
& \Pi\left(\theta_{1}^{*}, p \mid \text { dados }\right)=\int_{-\infty}^{\infty} \Pi\left(\theta_{1}^{*}, p, \beta \mid \text { dados }\right) d \beta \text { ou seja, } \\
& \Pi\left(\theta_{1}^{*}, p \mid \text { dados }\right)=\frac{p^{r+1}}{\left(\theta_{1}^{*}\right)^{p r+1}}(\Gamma(1+1 / p))^{p r} \prod_{i=1}^{k} \prod_{j=1}^{r_{i}} t_{i j}^{p-1} \\
& \times \int_{-\infty}^{\infty} \frac{\prod_{i=1}^{k} V_{i}^{\beta p r_{i}}}{V_{1}^{\beta p r}} \exp \left\{-\left(\frac{\Gamma(1+1 / p)}{\theta_{1}^{*}}\right)^{p} \sum_{i=1}^{k} A_{i}(p)\left(\frac{V_{i}}{V_{1}}\right)^{\beta p}\right\} d \beta
\end{aligned}
$$

Usando o Método de Laplace para aproximações de integrais (ver, por exemplo, Tierney \& Kadane, 1986), a densidade $\Pi\left(\theta_{1}^{*}, p \mid d a d o s\right)$ reescrita de forma adequada é dada por:

$$
\Pi\left(\theta_{1}^{*}, p \mid \text { dados }\right) \propto \frac{p^{r+1}}{\left(\theta_{1}^{*}\right)^{p r+1}}(\Gamma(1+1 / p))^{p r}\left(\prod_{i=1}^{k} \prod_{j=1}^{r_{i}} t_{i j}^{p-1}\right) \int_{-\infty}^{\infty} e^{-n h_{\theta_{1}^{*}, p}(\beta)} d \beta
$$

onde $\quad-n h_{\theta_{i}^{*}, p}(\beta)=\beta p \sum_{i=1}^{k} r_{i} \ln V_{i}-\beta p r \ln V_{1}-\left(\frac{\Gamma(1+1 / p)}{\theta_{i}^{*}}\right)^{p} \sum_{i=1}^{k} A_{i}(p)\left(\frac{V_{i}}{V_{1}}\right)^{\beta p}$

Assim, pelo Método de Laplace, a densidade a posteriori marginal conjunta para $\theta_{1}^{*}$ e p obtida é dada por: 


$$
\begin{aligned}
\Pi\left(\theta_{1}^{*}, p \mid \text { dados }\right) & \propto p^{r}\left(\theta_{1}^{*}\right)^{-p(r-1 / 2)-1}\left(\prod_{i=1}^{k} \prod_{j=1}^{r_{i}} t_{i j}^{p-1}\right)\left(\Gamma\left(1+\frac{1}{p}\right)\right)^{p^{\prime}(r-1 / 2)} \\
& \times \exp \left\{p \hat{\beta} \sum_{i=1}^{k} r_{i} \ln V_{i}-p \widehat{\beta} r \ln V_{1}-\left(\frac{\Gamma\left(1+\frac{1}{p}\right)}{\theta_{i}^{*}}\right)^{p} \sum_{i=1}^{k} A_{i}(p)\left(\frac{V_{i}}{V_{1}}\right)^{\hat{\beta} p}\right\} \\
& \times\left[\sum_{i=1}^{k} A_{i}(p)\left(\ln \frac{V_{i}}{V_{1}}\right)^{2}\left(\frac{V_{i}}{V_{1}}\right)^{\hat{\beta} p}\right]^{-1 / 2}
\end{aligned}
$$

onde $\theta_{1}^{*}>0, p>0$ e $\hat{\beta}$ é dado por:

$$
\left(\frac{\theta_{1}^{*}}{\Gamma\left(1+\frac{1}{p}\right)}\right)^{p}\left(\sum_{i=1}^{k} r_{i} \ln V_{i}-r \ln V_{1}\right)=\sum_{i=1}^{k} A_{i}(p)\left(\frac{V_{i}}{V_{1}}\right)^{\hat{\beta} p} \ln \left(\frac{V_{i}}{V_{1}}\right)
$$

Usando a densidade a posteriori conjunta para $\theta_{1}^{*}$ e p dada em (6.13), é possivel fazer inferências sobre os dois parâmetros $\theta_{1}^{*}$ e p. Por exemplo, é possível construir contornos de credibilidade para $\theta_{1}^{*}$ e $p$ ou ainda, determinar a moda a posteriori da densidade (6.13). 


\section{Capítulo 7}

\section{Dois Exemplos}

\subsection{Um Exemplo com a Distribuição Exponencial}

As análises clássica e Bayesiana discutidas nos Capítulos 2 e 3 são ilustradas neste capítulo, por meio de um exemplo com dados gerados (ver Achcar e Dias, 1990).

Usando o Modelo de Lei de Potência Inversa (2.2) com $\alpha=500, \beta=0.8 \mathrm{e}$ 5 níveis de stress $V_{i}, i=1, \ldots, 5$, uma amostra aleatória com distribuição Exponencial (2.1), de tamanho $n_{i}$ com $r_{i}$ falhas foi gerada para cada nível $V_{i}$. Os dados obtidos, que representam o tempo de sobrevivência dos itens testados, estão apresentados na tabela abaixo:

\begin{tabular}{|c|c|c|c|c|c|}
\hline i & $\overline{V_{i}}$ & $\bar{\theta}_{i}$ & $n_{i}$ & $r_{i}$ & Observações Não - Censuradas \\
\hline 1 & 10 & 79.24 & 30 & 5 & 68101214 \\
\hline 2 & 20 & 45.52 & 30 & 8 & 455688914 \\
\hline 3 & 30 & 32.90 & 30 & 12 & 23356778891017 \\
\hline 4 & 40 & 26.14 & 30 & 18 & 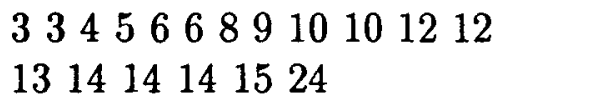 \\
\hline 5 & 50 & 21.87 & 30 & 22 & 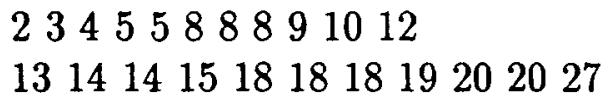 \\
\hline
\end{tabular}

Tabela 1: Dados gerados com $\alpha=500$ e $\beta=0.8$

O ajuste da distribuição Exponencial aos dados, é verificada através de uma análise gráfica baseada no estimador Produto Limite de Kaplan \& Meier. A escolha deste estimador não - paramétrico é justificada pelas suas boas propriedades. (ver Kaplan \& 
Meier, 1958).

A relação linear entre $\ln \left\{-\ln \widehat{S}\left(t_{i j}\right)\right\}$ e $\ln t_{i j}$ para $j=1, \ldots n_{i}$ e $i=1, \ldots, k$, onde $\widehat{S}(t)$ é o estimador Produto - Limite de Kaplan \& Meier, indica o possivel ajuste dos dados à distribuição Exponencial. Esta linearidade é verificada para as 5 amostras (ver Figuras $7.1-7.5$ ).

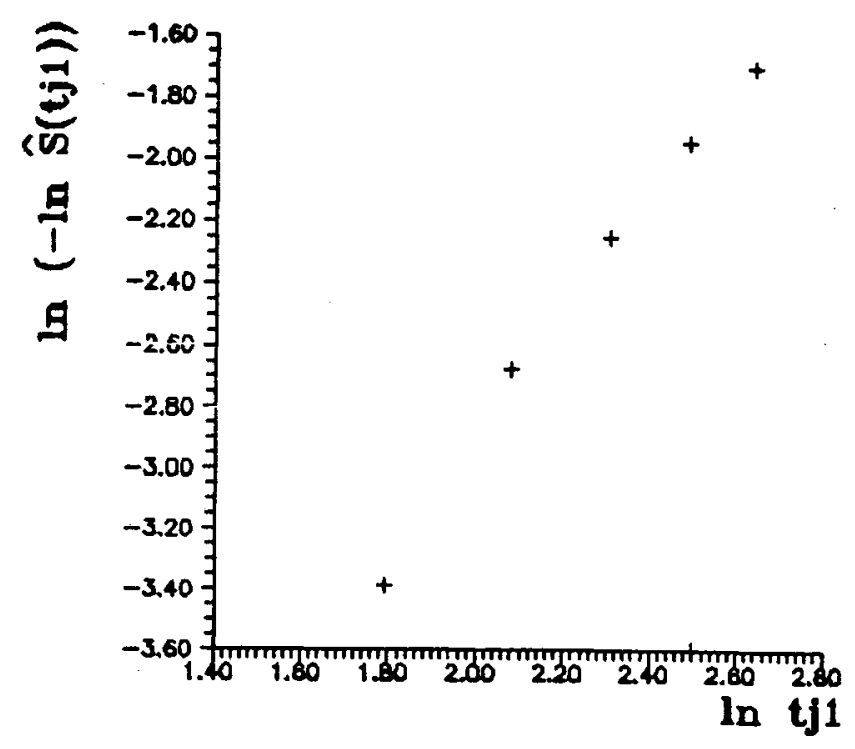

Figura 7.1: Amostra 1

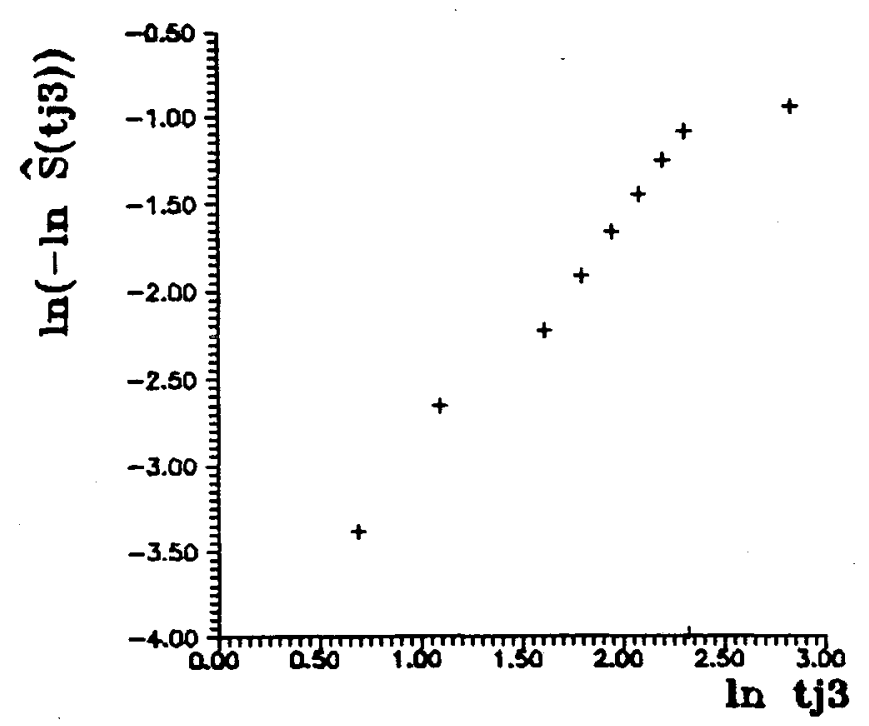

Figura 7.3: Amostra 3

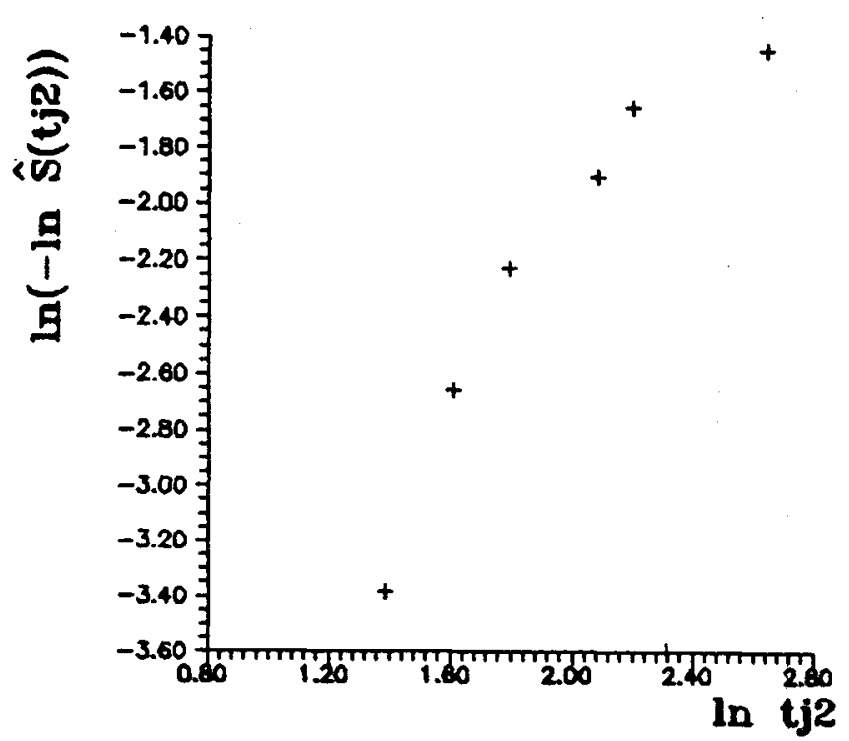

Figura 7.2: Amostra 2

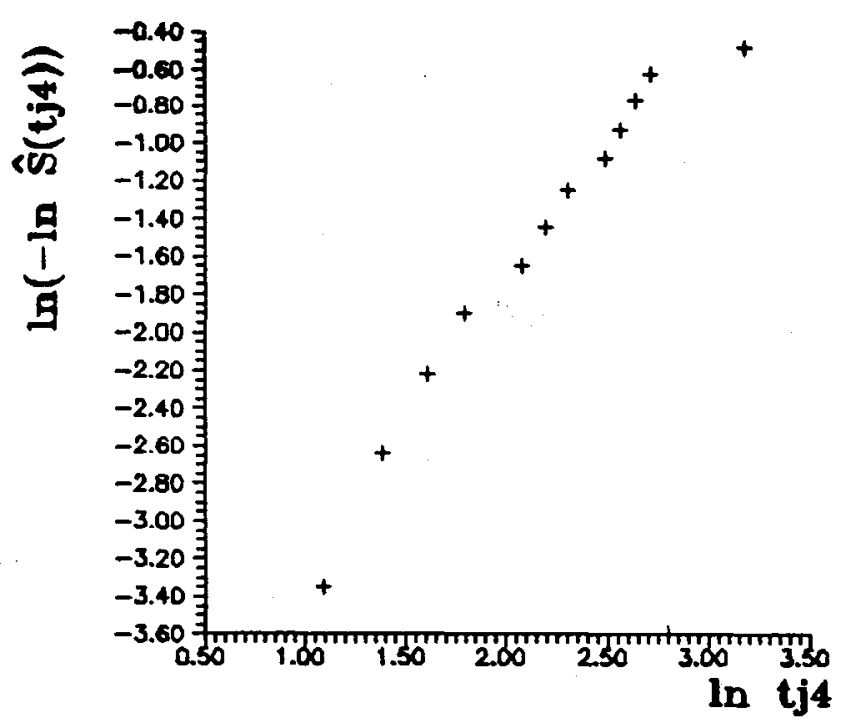

Figura 7.4: Amostra 4 


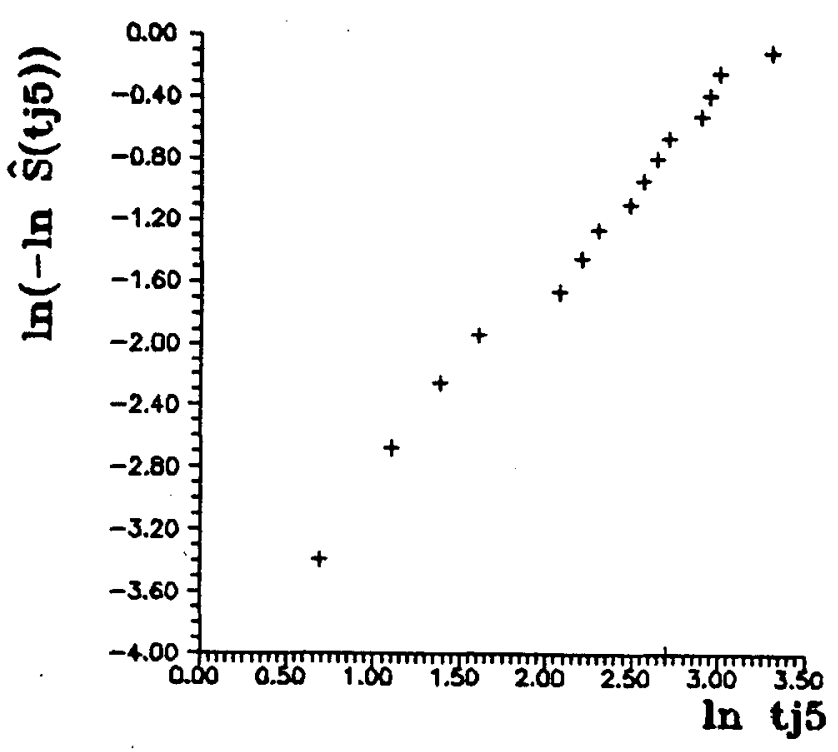

Figura 7.5: Amostra 5

Assumindo que os tempos gerados têm distribuição Exponencial, a adequabilidade do Modelo de Lei de Potência Inversa aos dados, é verificada da seguinte forma: de (2.2) tem-se que,

e a relação linear $\ln \lambda_{i}=-\ln \alpha+\beta \cdot \ln V_{i}$.

$$
\lambda_{i}=\frac{V_{i}^{\beta}}{\alpha},
$$

Considerando $\quad \hat{\lambda}_{i}=r_{i} / A_{i}$ o estimador de máxima verossimilhança para $\lambda_{i}$, para cada nível de stress, a relação linear entre $\ln \hat{\lambda}_{i}$ e $\ln V_{i}$ indica a adequabilidade do modelo (2.2) aos dados (ver Figura 7.6).

Para os dados $t_{i j}, j=1, \ldots, r_{i}$ e $i=1, \ldots, 5$ o logaritmo da função de verossimilhança para $\alpha$ e $\beta$ (ver 2.6), é dada por:

$$
l(\alpha, \beta)=228.7575 \beta-65 \ln \alpha-\frac{\sum_{i=1}^{5} A_{i} V_{i}^{\beta}}{\alpha}
$$

Os estimadores de máxima verossimilhança, (ver 2.7) são dados por:

$$
\left\{\begin{array}{l}
\hat{\alpha}=502.0524 \\
\widehat{\beta}=0.8003
\end{array}\right.
$$




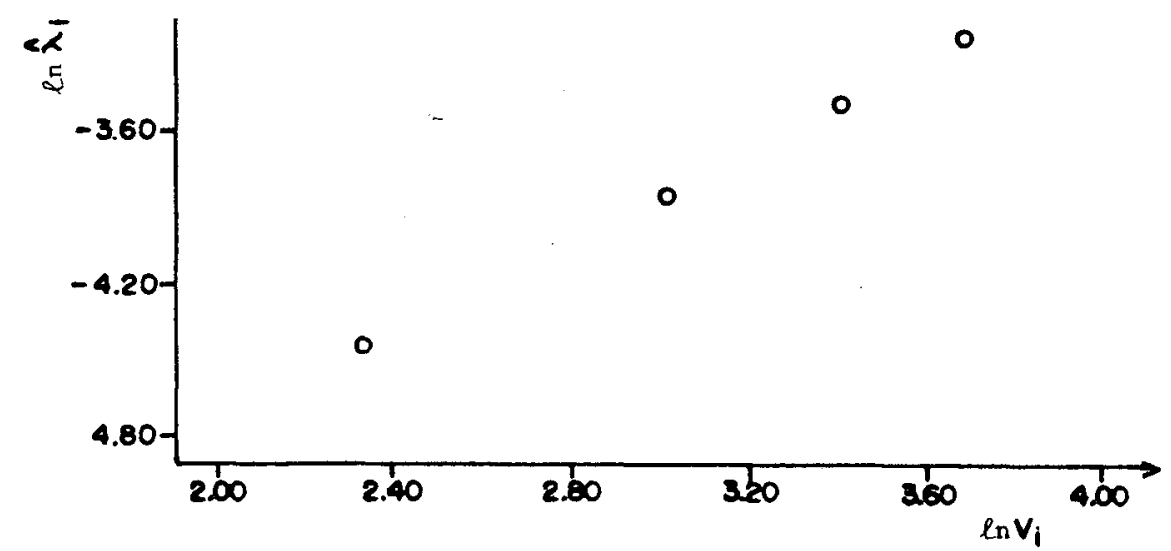

Figura 7.6: Gráfico de $\ln \left(\lambda_{i}\right)$ versus $\ln \left(V_{i}\right)$

O estimador de máxima verossimilhança para $\theta_{1}$ (tempo médio de sobrevivência sob as condições normais de uso) é dado por:

$$
\widehat{\theta}_{1}=\frac{\widehat{\alpha}}{V_{1}^{\widehat{\beta}}}=79.5150
$$

A matriz de Informação de Fisher para $\alpha$ e $\beta$ (ver (2.10)), calculada em $\widehat{\alpha}$ e $\hat{\beta}$, é dada por:

$$
I(\widehat{\alpha}, \widehat{\beta})=\left(\begin{array}{cc}
0.000258 & -0.455645 \\
-0.455645 & 818.749806
\end{array}\right)
$$

Usando o Resultado (2.2), os estimadores de máxima verossimilhança têm distribuição Normal Assintótica dada em (2.8). Assim,

$$
(\widehat{\alpha}, \widehat{\beta}) \stackrel{a}{\sim} N\left\{(\alpha, \beta) ; I^{-1}(\widehat{\alpha}, \widehat{\beta})\right\}
$$

onde $I^{-1}(\widehat{\alpha}, \widehat{\beta})$ é dada por:

$$
I^{-1}(\widehat{\alpha}, \widehat{\beta})=\left(\begin{array}{cc}
232204.6 & 129.22 \\
129.22 & 0.07314
\end{array}\right)
$$

A partir de (2.11), os intervalos com $90 \%$ de confiança para $\alpha$ e $\beta$ encontrados são dados por:

$$
i c(\alpha)=[-288.2244 ; 1292.3292]
$$




$$
i c(\beta)=[0.3568 ; 1.2438]
$$

e pelo Método Delta, usando a distribuição Normal Assintótica (2.13), um intervalo com $90 \%$ de confiança para $\theta_{1}$ dado em (2.14) é:

$$
i c\left(\theta_{1}\right)=[33.6654 ; 125.4033]
$$

Note que ao usar a Teoria da Aproximação Assintótica para os parâmetros $\alpha$ e $\beta$, o intervalo para $\alpha$ não respeita o intervalo de validade do parâmetro $(\alpha>0)$ sendo ainda muito grande, e pelo Método Delta, o intervalo encontrado para $\theta_{1}$ pode ser considerado grande.

Assumindo $\alpha$ e $\beta$ parâmetros desconhecidos e a priori de Jeffreys (3.1), a densidade a posteriori marginal para $\beta$ dada em (3.3) é:

$$
\Pi(\beta \mid \text { dados }) \propto \frac{\left\{2.23 \times 10^{99}\right\}^{\beta}}{\left\{400\left(10^{\beta}\right)+367\left(20^{\beta}\right)+391\left(30^{\beta}\right)+470\left(40^{\beta}\right)+486\left(50^{\beta}\right)\right\}^{65}}
$$

onde $-\infty<\beta<\infty$ (ver Figura 7.7).

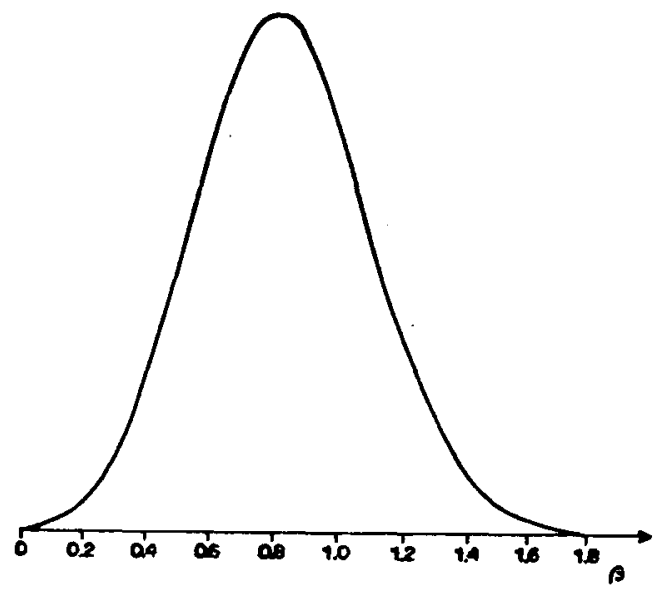

Figura 7.7: Densidade a Posteriori Marginal para $\beta$

O estimador pontual para $\beta$, a moda a posteriori, encontrada em (3.4), é dada por:

$$
\tilde{\beta} \cong 0.8
$$

A densidade a posteriori marginal para $\theta_{1}=\alpha / V_{1}^{\beta}$ dada em (3.7) usando o Método de Laplace para aproximação de integrais é mostrada na Figura 7.8. 


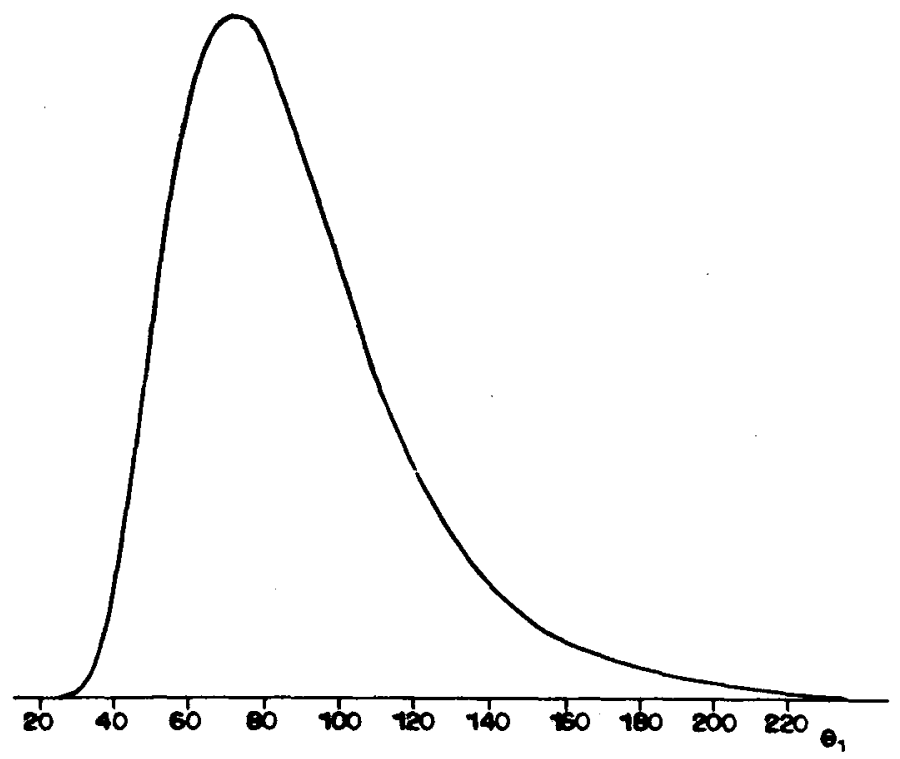

Figura 7.8: Densidade a Posteriori Marginal para $\theta_{1}$

Observando a Figura 7.8, uma reparametrização pode ser aplicada em $\theta_{1}$, para se obter uma forma conhecida da densidade $\Pi\left(\theta_{1} \mid d a d o s\right)$ e portanto, mais simples para se construir um intervalo de credibilidade para o parâmetro de interesse. Considerando a reparametrização $\delta_{1}=\ln \theta_{1}$, o gráfico da densidade a posteriori marginal para $\delta_{1}$, $\Pi\left(\delta_{1} \mid\right.$ dados $)$ (ver Apêndice $\left.C\right)$, mostrado na Figura 7.9, indica que através dessa transformação, a aproximação Normal para a posteriori de $\delta_{1}$ pode ser usada (ver Capítulo 2). Um intervalo com $90 \%$ de credibilidade para $\delta_{1}$ é dado por: $[3.7999 ; 4.9534]$.

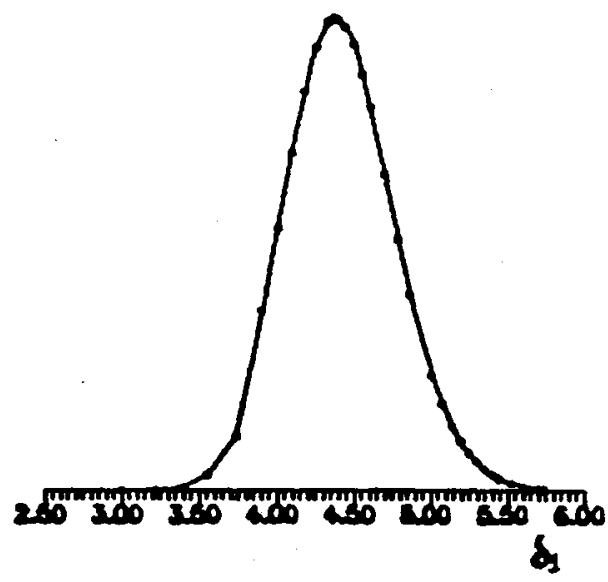

Figura 7.9: Densidade a Posteriori Marginal para $\delta_{1}=\ln \theta_{1}$ 
Para o parâmetro de interesse $\theta_{1}=e^{\delta_{1}}$, um intervalo com $90 \%$ de credibilidade é dado por [44.6967; 141.6558], que é diferente do intervalo [33.6654; 125.4033] encontrado usando a normalidade assintótica dos estimadores de máxima verossimilhança parâ $\alpha$ e $\beta$.

Uma análise gráfica no contorno da função de verossimilhança de $\alpha$ e $\beta$ mostra que esta aproximação não é boa (ver Figura 7.10). O contorno não apresenta uma forma elíptica, indicando que para este caso, a aproximação Normal para os estimadores $\widehat{\alpha}$ e $\widehat{\beta}$ não é adequada.

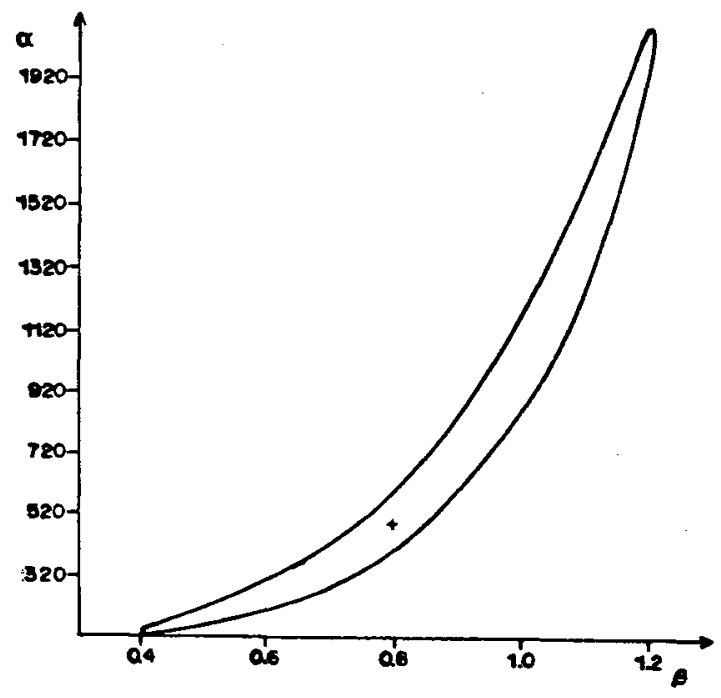

Figura 7.10: Contorno da Função de Verossimilhança para $\alpha$ e $\beta$

Assumindo $\beta$ conhecido, ou seja $\beta=0.8$, a forma da densidade a posteriori marginal para $\theta_{1}$ dada em (3.9) é mostrada na Figura 7.11. Então, usando (3.11), o intervalo com $90 \%$ de credibilidade encontrado é dado por: [65.6824 ; 98.9963]. A moda a posteriori da densidade para $\theta_{1}$ dada em (3.10) é:

$$
\tilde{\theta}_{1}=78.28
$$

O intervalo de credibilidade para $\theta_{1}$, assumindo $\beta$ conhecido, tem comprimento $L_{1}=33.3199$.

É possível aplicar os resultados do Capítulo 4 neste exemplo, para planejar um experimento com testes acelerados. Supor que o pesquisador tenha interesse em obter um comprimento do intervalo com $90 \%$ de credibilidade dado por $L=32$ (isto é, menor do que $L_{1}=33.3199$ obtido acima). Assim, ele quer estimar o número necessário de unidades num nivel adicional de stress, $V_{6}=60$. 


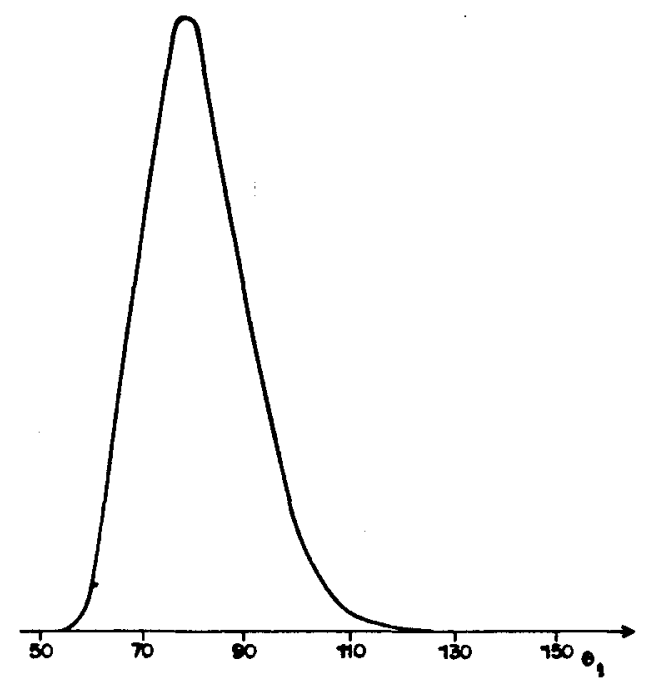

Figura 7.11: Densidade a Posteriori Marginal para $\theta_{1} \operatorname{com} \beta$ conhecido

De (4.6) com $r=65,1-\gamma=0.90$ e $L=32$ fixados, é possível encontrar valores de $1-\gamma^{*}$ para vários valores de $r_{6}$ (número de falhas no nível $V_{6}=60$ ). Estes valores são dados na Tabela 2.

\begin{tabular}{|c|c|}
\hline$r_{6}$ & $1-\gamma^{*}$ \\
\hline 2 & 0.06 \\
3 & 0.30 \\
4 & 0.45 \\
5 & 0.54 \\
6 & 0.62 \\
7 & 0.65 \\
8 & 0.75 \\
9 & 0.78 \\
10 & 0.81 \\
11 & 0.84 \\
12 & 0.87 \\
13 & 0.90 \\
14 & 0.91 \\
15 & 0.92 \\
16 & 0.94 \\
17 & 0.95 \\
\hline \hline
\end{tabular}

Tabela 2: Confiança observada $\left(1-\gamma^{*}\right)$ para diferentes valores de $r_{6}$ 
Assim, observa-se que se $1-\gamma^{*}=0.90$ é fixado, o pesquisador precisa acrescentar $r_{6}=13$ unidades no nivel adicional de stress $V_{6}=60$.

Considere os resultados obtidos no Capítulo 5 e suponha $\beta$ conhecido, ou seja $\beta=0.8$ (ver Capítulo 3 , secção 3.2). Neste exemplo, a função densidade preditiva para uma observação futura em um nivel i de stress $\mathrm{V}$, dada em (1.4), é:

$$
f^{i}\left(t_{(n+1) i} \mid t_{1}, \ldots, t_{n}\right)=\frac{65 V_{i}^{0.8}(32598.967)^{65}}{\left\{V_{i}^{0.8} t_{(n+1) i}+32598.967\right\}^{66}}, \quad t_{(n+1) i}>0 .
$$

As expressões de esperança e variância de $T_{(n+1) i}$, dadas em (1.7) e (1.8) respectivamente, são dadas por:

$$
\begin{aligned}
E^{i}\left(T_{(n+1) i} \mid t_{1}, \ldots, t_{n}\right) & =\frac{32598.967}{64 V_{i}^{0.8}} \\
\operatorname{Var}^{i}\left(T_{(n+1) i} \mid t_{1}, \ldots, t_{n}\right) & =\frac{65(32598.967)^{2}}{(64)^{2}(63) V_{i}^{1.6}}
\end{aligned}
$$

Para os 5 níveis de stress assumidos neste exemplo, os momentos de $f^{i}\left(t_{(n+1) i} \mid t_{1}, \ldots, t_{n}\right)$ são dados na Tabela 3.

\begin{tabular}{|lllll|}
\hline$i$ & $V_{i}$ & $E^{i}\left(T_{(n+1) i} \mid\right.$ dados $)$ & $\operatorname{Var}^{i}\left(T_{(n+1) i} \mid\right.$ dados $)$ & $\sqrt{\operatorname{Var}^{i}\left(T_{(n+1) i} \mid \text { dados }\right)}$ \\
\hline 1 & 10 & 80.7279 & 6723.8890 & 81.9993 \\
2 & 20 & 46.3660 & 2218.0562 & 47.0962 \\
3 & 30 & 33.5218 & 1159.3819 & 34.0497 \\
4 & 40 & 26.6303 & 731.6857 & 27.0497 \\
5 & 50 & 22.2765 & 511.9983 & 22.6274 \\
\hline \hline
\end{tabular}

Tabela 3: Momentos da Variável $T_{(n+1) i}$

Assumindo $\beta=0.8$ conhecido, pela Tabela 1 tem - se que $r=65$ e $C=\sum_{j=1}^{5} A_{j} V_{j}^{0.8}=32598.967$.

Considerando $1-\gamma=0.80$ e $V_{i}=30, P_{i}$ encontrado (de (5.12)) é dado por $P_{i}=7.3777$. Num teste de controle de qualidade, $m$ unidades são colocadas num nível de stress $V_{i}=30$ durante o período de tempo $P_{i}=7.38$ e observa - se o número $\mathrm{X}$ de falhas. A linha de produção está fora de controle se a hipótese $H_{0}: \gamma^{i} \leq 0.20$ for rejeitada, onde $\gamma^{i}=P_{i}\left\{T_{(n+1) i} \leq P_{i} \mid\right.$ dados $\}$ num nivel de significância estipulado $\alpha$. Isto é, a linha de produção está fora de controle se $X \geq 0.20 m+0.40 Z_{\alpha} \sqrt{m}$. 
Outra possibilidade é fixar um periodo de tempo $P_{i}=20,1-\gamma=0.80 \mathrm{e}$ encontrar um nivel de stress $V_{i}$ (de (5.13) ) dado por $V_{i}=8.6246$.

Observar que o critério descrito para controle de qualidade é muito flexível em termos das escolhas de $V_{i}$ e $P_{i}$ para minimizar tempo e custo dos testes (ver Achcar, 1990).

\subsection{Um Exemplo com a Distribuição de Weibull}

Os resultados obtidos no Capítulo 6 referente à análise Bayesiana, são aplicados, nesta seç̧ão, em um conjunto de dados (ver Tabela 4) sob um teste de sobrevivência acelerado, introduzido por Nelson (1972). Esses dados são supostos terem distribuição de Weibull (6.1), e a relação entre o parâmetro da distribuição de vida $\lambda^{*}$ e a variável stress $V$, é dada pelo Modelo de Lei de Potência Inversa (6.2). Isto foi verificado graficamente por Nelson (1972).

Os dados (ver Tabela 4), representam os tempos de ruptura de um material isolante, e são considerados sete níveis de voltagem.

\begin{tabular}{|c|ll|l|l|}
\hline $\mathrm{i}$ & $V_{i}$ & $n_{i}$ & \multicolumn{1}{|c|}{ Tempos Observados } \\
\hline 1 & 26 & 3 & 5.791579 .522323 .70 \\
2 & 28 & 5 & 68.85108 .29110 .29426 .071067 .60 \\
3 & 30 & 11 & 7.7417 .0520 .4621 .0222 .6643 .4047 .30 \\
& & & 139.07144 .12175 .88194 .90 \\
4 & 32 & 15 & 0.270 .400 .690 .792 .753 .919 .8813 .95 \\
& & & 15.9327 .8053 .2482 .8589 .29100 .58215 .10 \\
5 & 34 & 19 & 0.190 .780 .961 .312 .783 .164 .154 .67 \\
& & & 4.856 .507 .358 .018 .2712 .0631 .7532 .52 \\
& & & 33.9136 .7172 .89 \\
6 & 36 & 15 & 0.350 .590 .960 .991 .691 .972 .072 .58 \\
& & & 2.712 .903 .673 .99 & 5.3513 .7725 .50 \\
7 & 38 & 8 & 0.090 .390 .470 .730 .741 .131 .402 .38 \\
\hline \hline
\end{tabular}

Tabela 4: Tempos de vida de um material isolante

Note que este conjunto de dados foi coletado usando um esquema de amostragem completa. Ou seja, considerando $\mathrm{k}$ níveis de uma variável stress $V_{i}, i=1,2, ., k$ onde os tempos de sobrevivência têm distribuiçẫo de Weibull, uma amostra de $n_{\boldsymbol{i}}$ unidades é 
colocada em teste, para cada nível $V_{i}$. O experimento termina quando todas as observações falham.

Para os tempos de sobrevivência $t_{i j}, j=1 \ldots, n_{i}$ e $i=1, \ldots, 7$, o logaritmo da função de verossimilhança (6.5) é:

$$
\begin{aligned}
l(\alpha, \beta, p) & =76 \ln p-76 p \ln \alpha+265.68884 \beta p \\
& +(p-1) \sum_{i=1}^{7} \sum_{j=1}^{n_{i}} \ln t_{i j}-\frac{\sum_{i=1}^{7} V_{i}^{\beta_{p}} A_{i}(p)}{\alpha^{p}}
\end{aligned}
$$

onde $A_{i}(p)=\sum_{j=1}^{n_{i}} t_{i j}^{p}$ ( considerando o esquema de amostras completas).

Considerando a priori de Jeffreys (6.6), a densidade a posteriori marginal para p (dada em (6.10)) aproximada pelo Método de Laplace, é dada por,

$$
\Pi(p \mid \text { dados }) \propto \frac{(76 p+1)^{75}\left(\prod_{i=1}^{7} V_{i}^{\hat{\beta}_{p n_{i}}}\right)\left(\prod_{i=1}^{7} \prod_{j=1}^{n_{i}} t_{i j}^{p-1}\right) e^{-(76 p+1) / p}}{\left(\sum_{i=1}^{7} V_{i}^{\hat{\beta}_{p}} A_{i}(p)\right)^{76} B(p, \hat{\beta})},
$$

onde $p>0$ e $B(p, \hat{\beta})$ é dada em (6.11) (ver Figura 7.12).

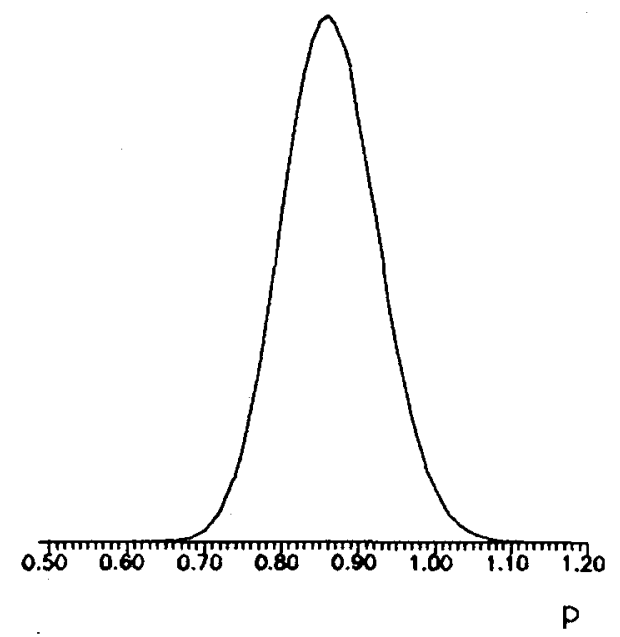

Figura 7.12: Densidade a Posteriori Marginal para $\mathrm{p}$ 
Observando a Figura 7.12, pode-se verificar que a moda da densidade a posteriori para p, é dada por:

$$
\tilde{p}=0.86
$$

e um intervalo com $95 \%$ de credibilidade para $p$ é dado por: $[0.74 ; 0.99]$

Assumindo $\mathrm{p}$ conhecido através da moda $\tilde{p}$, os resultados da análise Bayesiana, vistas no Capítulo 3 , podem ser considerados, pois os dados $t_{i j}^{\tilde{p}}, i=1, \ldots, k$ e $j=1, \ldots, n_{i}$ têm distribuição Exponencial.

Assim, para os tempos de sobrevivência $t_{i j}^{0.86}, i=1, \ldots, 7$ e $j=1, \ldots, n_{i}$, o logaritmo da função de verossimilhança para $\alpha$ e $\beta,(2.6)$, é dado por:

$$
l(\alpha, \beta)=265.68884 \beta-76 \ln \alpha-\frac{\sum_{i=1}^{7} A_{i} V_{i}^{\beta}}{\alpha}
$$

Assumindo $\alpha$ e $\beta$ desconhecidos e a priori de Jeffreys (3.1), a densidade a posteriori marginal para $\beta$, ver (3.3) é dada por:

$$
\Pi(\beta \mid d a d o s) \propto \frac{\prod_{i=1}^{7} V_{i}^{\beta n_{i}}}{\left(\sum_{i=1}^{7} A_{i} V_{i}^{\beta}\right)^{76}}, \quad-\infty<\beta<\infty
$$

O gráfico de $\Pi(\beta \mid$ dados $)$ é mostrado na Figura 7.13.

A moda da densidade a posteriori para $\beta$ dada na Figura (7.13), é dada por:

$$
\tilde{\beta} \cong 15.01
$$

A densidade a posteriori marginal para $\theta_{1}=\alpha / V_{1}^{\beta}$ dada em (3.7) (ver Figura 7.14), usando o Método de Laplace para aproximações de integrais, é dada por:

$$
\Pi\left(\theta_{1} \mid \text { dados }\right) \propto \frac{\left[h^{\prime \prime}(\hat{\beta})\right]^{-\frac{1}{2}} \prod_{i=1}^{7} V_{i}^{\hat{\beta}_{n_{i}}}}{\left(\theta_{1}^{77}\right)\left(26^{76} \hat{\beta}\right)} \exp \left\{\frac{-\sum_{i=1}^{7} A_{i} V_{i}^{\hat{\beta}}}{\theta_{1} 26 \hat{\beta}}\right\}, \quad \theta_{1}>0
$$

Observando a Figura 7.14, uma reparametrização pode ser aplicada. De maneira análoga ao Exemplo 1 , considere a reparametrização $\delta_{1}=\ln \theta_{1}$ (ver Apêndice $C$ ). 
O gráfico da densidade a posteriori marginal para $\delta_{1}, \Pi\left(\delta_{1} \mid\right.$ dados $)$, mostrado na Figura 7.15 , indica que a aproximação Normal para a posteriori de $\delta_{1}$ pode ser usada.

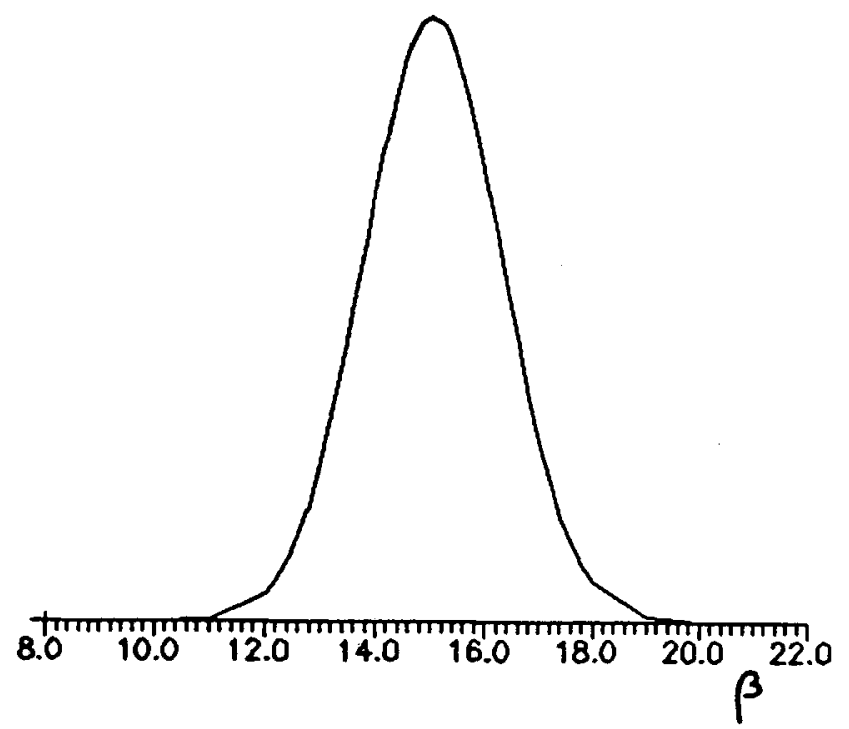

Figura 7.13: Densidade a Posteriori Marginal para $\beta$

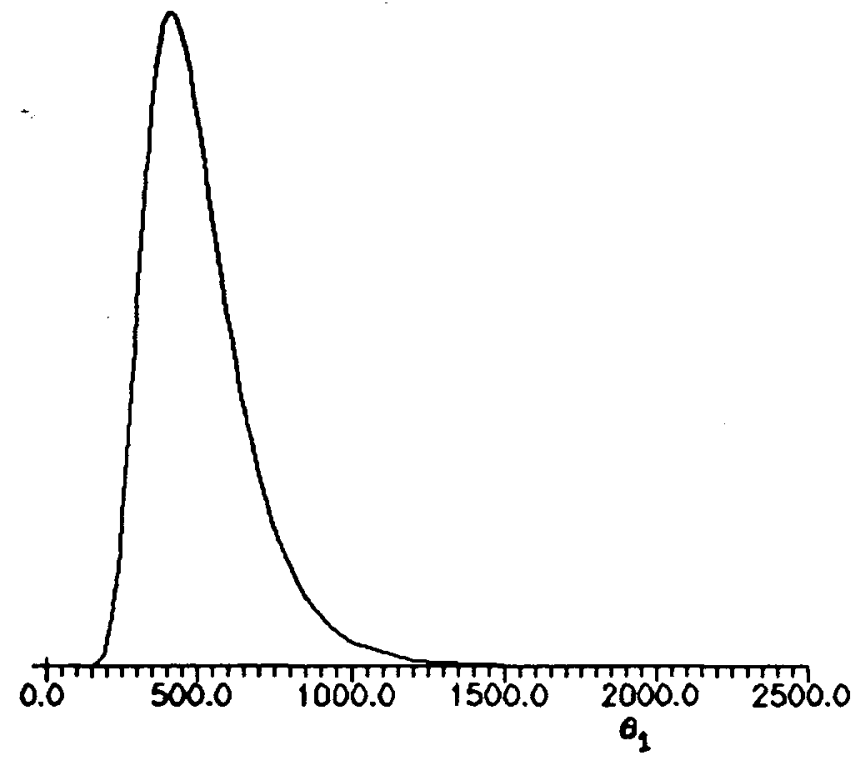

Figura 7.14: Densidade a Posteriori Marginal para $\theta_{1} \operatorname{com} \beta$ desconhecido 


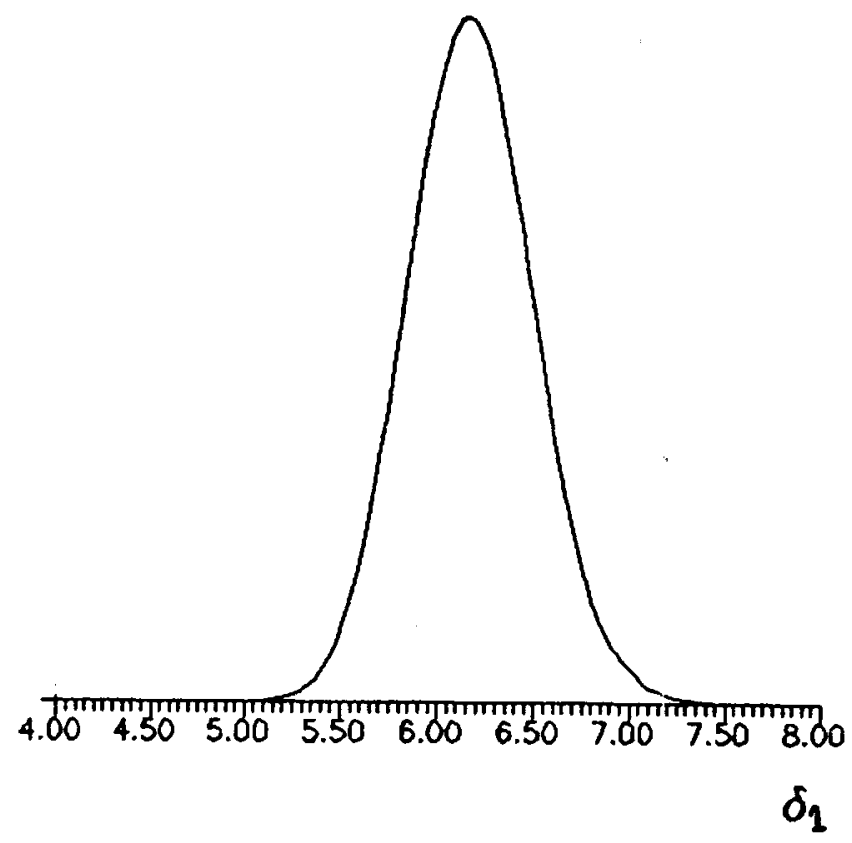

Figura 7.15: Densidade a Posteriori Marginal para $\delta_{1}=\ln \theta_{1}$

Um intervalo com $90 \%$ de credibilidade para $\delta_{1}$ é dado por: $[5.60714 ; 6.60675]$. Para $\theta_{1}=e^{\delta_{1}}$ como parâmetro de interesse, um intervalo com $90 \%$ de credibilidade para $\theta_{1}$, é dado por: [272.36416; 740.07386]. Observar que $\theta_{1}$ é o tempo médio de sobrevivência para os itens submetidos ao nível usual de stress $V_{1}=26$, considerando os dados transformados $t_{i j}^{0.86}, i=1, \ldots, 7$ e $j=1, \ldots, n_{i}$.

\subsubsection{Densidade a Posteriori Conjunta para $\theta_{1}^{*}$ e p: $\Pi\left(\theta_{1}^{*}, p \mid\right.$ dados $)$}

Nesta subsecção, considerando os resultados da secção 6.2 , é possível analisar o comportamento dos parâmetros $\theta_{1}^{*}$ e $\mathrm{p}$, conjuntamente, através da densidade $\Pi\left(\theta_{1}^{*}, p \mid\right.$ dados $)$.

A densidade a posteriori conjunta para $\theta_{1}^{*}, \beta$ e p, dada em (6.12) é:

$$
\begin{aligned}
\Pi\left(\theta_{1}^{*}, \beta, p \mid \text { dados }\right) & \propto \frac{p^{n+1}}{\left(\theta_{i}^{*}\right)^{p n+1}}\left(\frac{\Gamma(1+1 / p)}{26^{\beta}}\right)^{p}\left(\prod_{i=1}^{7} V_{i}^{\beta p n_{i}}\right)\left(\prod_{i=1}^{7} \prod_{j=1}^{n_{i}} t_{i j}^{p-1}\right) \\
& \times \exp \left\{-\left(\frac{\Gamma(1+1 / p)}{\theta_{i}^{*}}\right)^{p} \sum_{i=1}^{7} A_{i}(p)\left(\frac{V_{i}}{26}\right)^{\beta}\right\}
\end{aligned}
$$

$\operatorname{para} \theta_{1}^{*}>0, p>0$ e $-\infty<\beta<\infty$ 
A densidade a posteriori marginal para $\theta_{1}^{*}$ e $p$, reescrita de forma conveniente, usando o Método de Laplace (ver Apêndice B) é dada por:

$$
\begin{aligned}
\Pi\left(\theta_{1}^{*}, p \mid \text { dados }\right) & \propto \frac{p^{77}}{\left(\theta_{1}^{*}\right)^{76 p+1}}\left[\Gamma\left(1+\frac{1}{p}\right)\right]^{76 p}\left[\prod_{i=1}^{7} \prod_{j=1}^{n_{i}} t_{i j}^{p-1}\right] \\
& \times \int_{-\infty}^{\infty} e^{-n h_{\theta_{1}^{*}, p}(\beta)} d \beta
\end{aligned}
$$

onde $-n h_{\theta_{i}^{*}, p}(\beta)=18.07350 \beta p-\left(\frac{\Gamma(1+1 / p)}{\theta_{i}^{*}}\right)^{p} \sum_{i=1}^{7} A_{i}(p)\left(\frac{V_{i}}{26}\right)^{\beta p}$

e $\hat{\beta}$ que maximiza $-n h(\beta)$ é tal que satisfaça:

$$
18.07350\left(\frac{\theta_{1}^{*}}{p\left(1+\frac{1}{p}\right)}\right)^{p}=\sum_{i=1}^{7} A_{i}(p)\left(\frac{V_{i}}{26}\right)^{\hat{\beta}_{p}}\left(\ln \left(\frac{V_{i}}{26}\right)\right)
$$

Portanto,

$$
\begin{aligned}
\Pi\left(\theta_{1}^{*}, p \mid \text { dados }\right) & \propto \frac{p^{77}}{\left(\theta_{1}^{*}\right)^{76 p+1}}\left(\Gamma\left(1+\frac{1}{p}\right)\right)^{76 p}\left(\prod_{i=1}^{7} \prod_{j=1}^{n_{i}} t_{i j}^{p-1}\right) \\
& \times\left(n h^{\prime \prime}(\hat{\beta})\right)^{-\frac{1}{2}} \exp \left\{\hat{\beta} p \sum_{i=1}^{7} n_{i} \ln V_{i}-247.61534 \hat{\beta} p\right. \\
& \left.-\left(\frac{\Gamma\left(1+\frac{1}{p}\right.}{\left(\theta_{i}^{*}\right)}\right)^{p} \sum_{i=1}^{7} A_{i}(p)\left(\frac{V_{i}}{26}\right)^{\beta_{p}}\right\}, \quad \theta_{1}^{*}>0 \text { e } p>0
\end{aligned}
$$

ou seja,

$$
\begin{aligned}
\Pi\left(\theta_{1}^{*}, p \mid \text { dados }\right) & \propto p^{76}\left(\theta_{1}^{*}\right)^{-p\left(\frac{75}{2}\right)-1}\left(\Gamma\left(1+\frac{1}{p}\right)\right)^{p \frac{75}{2}}\left(\prod_{i=1}^{7} \prod_{j=1}^{n_{i}} t^{p-1}\right) \\
& \times\left[\sum_{i=1}^{7} A_{i}(p)\left(\ln \frac{V_{i}}{26}\right)^{2}\left(\frac{V_{i}}{26}\right)^{\beta_{p}}\right]^{-\frac{1}{2}} \exp \left\{\hat{\beta} p \sum_{i=1}^{7} r_{i} \ln V_{i}\right. \\
& \left.-247.6153 \hat{\beta} p-\left(\frac{\Gamma\left(1+\frac{1}{p}\right)}{\theta_{i}^{*}}\right)^{p} \sum_{i=1}^{7} A_{i}(p)\left(\frac{V_{i}}{26}\right)^{\beta_{p}}\right\}
\end{aligned}
$$

$(\operatorname{ver}(6.13))$. 
Uma aproximação para o cálculo da função Gama, $\Gamma(Z)$, onde $Z \in \Re$ é dada em Abramowitz \& Stegun (1968). Na Figura 7.19, tem-se alguns contornos para os parâmetros $\ln \theta_{1}^{*}$ e $\ln p$ obtidos a partir da densidade a posteriori conjunta de $\ln \theta_{1}^{*}$ e $\ln p$ (calculada a partir da posteriori conjunta $\Pi\left(\theta_{1}^{*}, p \mid\right.$ dados)). É possivel, usar a moda da posteriori de $\ln \theta_{1}^{*}$ e $\ln p$ para ser considerada como estimadores pontuais de $\ln \theta_{1}^{*}$ e $\ln p$ e assim, de $\theta_{1}^{*}$ e p. Também é possível encontrar intervalos de credibilidade para $\theta_{1}^{*}$ e p, onde $\theta_{1}^{*}$ é o tempo médio de sobrevivência dos itens submetidos ao nível usual de stress $V_{1}=26$ com os dados na escala original.

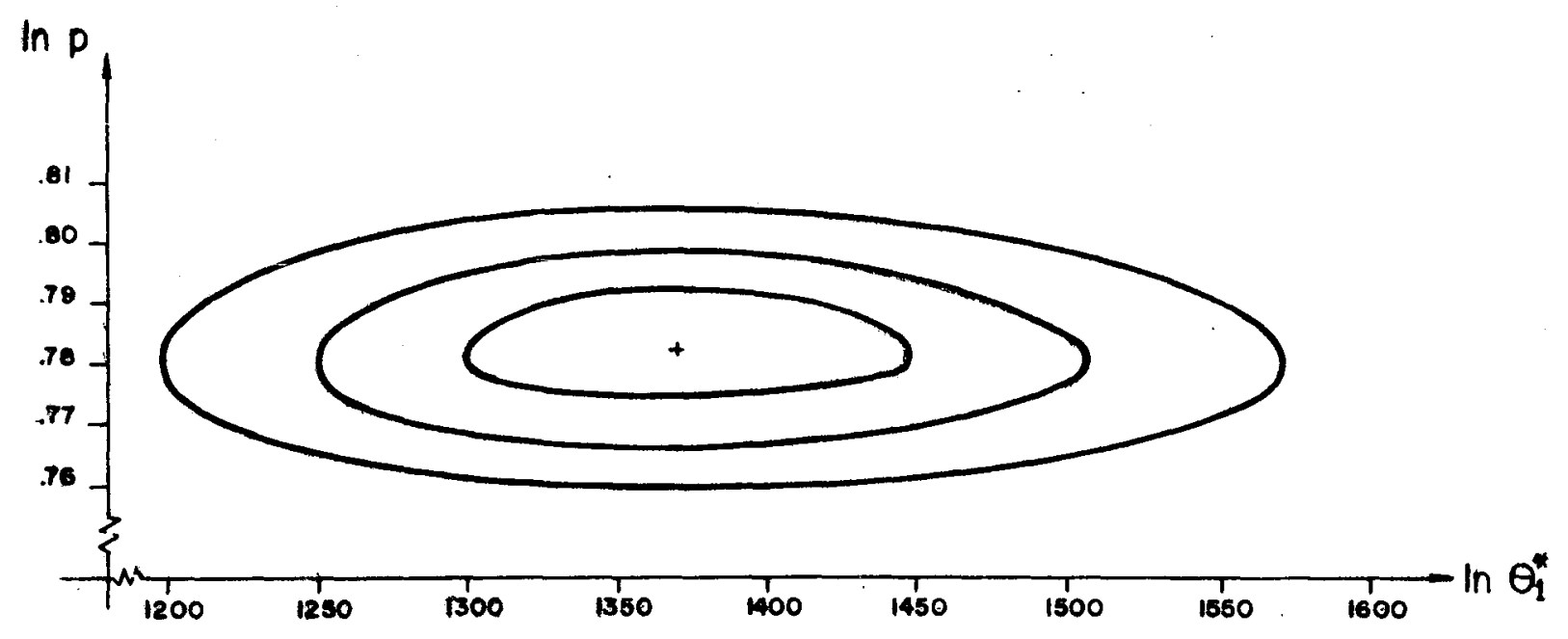

Figura 7.16: Contornos para a Densidade a Posteriori Conjunta para $\ln \theta_{1}^{*}$ e $\ln \mathrm{p}$ 


\section{Capítulo 8}

\section{Considerações Futuras}

Neste estudo, considerando um experimento onde os tempos de sobrevivência têm distribuição Exponencial assumindo um mecanismo de censura do Tipo II, análises clássica e Bayesiana foram desenvolvidas. $\mathrm{Na}$ análise Bayesiana, partindo de uma priori não - informativa de Jeffreys, densidades a posteriori marginais para os parâmetros de interesse foram determinadas, usando o Método de Laplace. Uma análise Bayesiana também foi desenvolvida para tempos de sobrevivência com distribuição de Weibull.

Com a aplicação de um método para aproximação de integrais, expressões mais simples foram obtidas para as densidades.

Como seqüência para a pesquisa desenvolvida nesta dissertação de mestrado, alguns itens podem ser destacados para serem estudados num estudo futuro:

1. O uso de métodos de aproximação para integrais requer muito cuidado quanto à precisão dos resultados obtidos. Achcar \& Smith (1990), estudaram o efeito de reparametrização para os métodos de Laplace e Família de Pearson e através de exemplos, comparam a precisão desses métodos com as reparametrizações adotadas.

O " problema" de aproximar integrais pelo Método de Laplace, é que resultados precisos são obtidos quando a forma de densidade se aproxima de uma distribuição Normal. Para amostras pequenas ou moderadas e em particular quando os parâmetros são definidos em intervalos diferentes da reta real, é necessário tomar cuidado na aplicação deste método. $\mathrm{O}$ método de aproximação baseado na Família de. Pearson, permite usar distribuições tais como as distribuições Gama ou Beta, porém estudada apenas para o caso uniparamétrico. 
Estes aspectos motivam um estudo mais detalhado sobre aproximações de integrais considerando diferentes reparametrizações, comparando os resultados obtidos quando à precisão do método aplicado com alguns métodos numéricos (por exemplo, o Método de Simpson). Também, outras distribuições de probabilidade podem ser consideradas.

2. O uso de outras densidades a priori para os parâmetros de interesse. Em aplicações industriais, é comum o uso de densidades a priori informativas em lugar das densidades a priori não - informativas, utilizada neste trabalho, pois muitas vezes os engenheiros têm informações sobre o mecanismo da distribuição dos parâmetros de interesse. Por exemplo, uma possível priori a ser estudada, pode ser dada pela priori conjugada.

3. O desenvolvimento de software para ser usado nas aplicações de testes acelerados nas indústrias.

4. Considerar aplicações do método Bayesiano proposto utilizando dados reais das indústrias. 


\section{APÊNDICES}

\section{Apêndice A \\ Método Delta}

Seja X uma variável aleatória com média $\mu$ e variância $\sigma^{2}$, denotada por:

$$
X \sim\left\{\mu ; \sigma^{2}\right\}
$$

e seja $g(X)$ uma função de $X$. Expandindo $g(X)$ sobre $\mu$ e desprezando as derivadas de ordem superior, tem-se que:

$$
g(X)=g(\mu)+(X-\mu) g^{\prime}(\mu)
$$

então, $\mathrm{g}(\mathrm{X})$ terá média e variância dadas por: $g(X) \approx\left\{g(\mu) ; \sigma^{2}\left(g^{\prime}(\mu)\right)^{2}\right\}$

Além disso, se $X \stackrel{a}{\sim}$ Normal, então, $g(X) \stackrel{a}{\sim}$ Normal.

Para o caso multivariado, em particular para os parâmetros $\hat{\alpha}$ e $\widehat{\beta}$, tem-se que:

$$
(\widehat{\alpha}, \widehat{\beta}) \sim\left\{(\alpha, \beta) ; I^{-1}(\widehat{\alpha}, \widehat{\beta})\right\}
$$

onde $I^{-1}(\widehat{\alpha}, \widehat{\beta})$ é a Matriz de Variância e Covariância dada em (2.9) .

Expandindo $g(\hat{\alpha}, \widehat{\beta})$ sobre $\alpha$ e $\beta$, tem-se que:

$$
g(\widehat{\alpha}, \widehat{\beta})=g(\alpha, \beta)+(\hat{\alpha}-\alpha)\left[\frac{\partial g(\alpha, \beta)}{\partial \alpha}\right]^{2}+2 \sigma_{\alpha \beta}\left[\frac{\partial g}{\partial \alpha}\right]\left[\frac{\partial g}{\partial \beta}\right]+(\widehat{\beta}-\beta)\left[\frac{\partial g(\alpha, \beta)}{\partial \beta}\right]^{2}
$$



dadas por:

Da mesma maneira que para o caso univariado, $g(\widehat{\alpha}, \widehat{\beta})$ terá média e variância

$$
g(\widehat{\alpha}, \widehat{\beta}) \simeq\left\{g(\alpha, \beta) ; \sigma_{\alpha}^{2}\left[\frac{\partial g}{\partial \alpha}\right]^{2}+2 \sigma_{\alpha \beta}\left[\frac{\partial g}{\partial \alpha}\right]\left[\frac{\partial g}{\partial \beta}\right]+\sigma_{\beta}^{2}\left[\frac{\partial g}{\partial \beta}\right]^{2}\right\}
$$

Usando o resultado (2.2), do Capítulo 2:

$$
(\hat{\alpha}, \widehat{\beta}) \stackrel{a}{\sim} N\left\{(\alpha, \beta) ; I^{-1}(\widehat{\alpha}, \widehat{\beta})\right\}
$$

Logo,

$$
g(\widehat{\alpha}, \widehat{\beta}) \stackrel{a}{\sim} N\left\{g(\alpha, \beta) ; \sigma_{\alpha}^{2}\left(\frac{\partial g}{\partial \alpha}\right)^{2}+2 \sigma_{\alpha \beta}\left(\frac{\partial g}{\partial \alpha}\right)\left(\frac{\partial g}{\partial \beta}\right)+\sigma_{\beta}^{2}\left(\frac{\partial g}{\partial \beta}\right)^{2}\right\}
$$




\section{Apêndice B}

\section{Aproximação de Integrais pelo Método de Laplace}

Em problemas onde a densidade a posteriori conjunta para um vetor de parâmetros é conhecida, e há interesse em se fazer inferências a respeito de um parâmetro particular, o Método de Laplace fornece resultados com boa precisão usando aproximações de integrais para achar a densidade a posteriori marginal e momentos a posteriori para o parâmetro de interesse.

Seja a densidade a posteriori conjunta $\Pi\left(\theta_{1}, \ldots, \theta_{p} \mid\right.$ dados $)$ e $\theta_{1}$ o parâmetro de interesse. A densidade a posteriori marginal para $\theta_{1}$ dada por:

$$
\Pi\left(\theta_{1} \mid \text { dados }\right)=\int \Pi\left(\theta_{1}, \ldots, \theta_{p} \mid d a d o s\right) d \theta_{2} \ldots d \theta_{p}
$$

A densidade a posteriori marginal $\Pi\left(\theta_{1} \mid\right.$ dados $)$ pode ser reescrita na forma:

$$
\Pi\left(\theta_{1} \mid d a d o s\right)=\int e^{-n h_{\theta_{1}}\left(\theta_{2}, \ldots, \theta_{p}\right)} d \theta_{2} \ldots d \theta_{p}
$$

onde $n h_{\theta_{1}}\left(\theta_{2}, \ldots, \theta_{p}\right)=\ln \Pi\left(\theta_{1}, \ldots, \theta_{p} \mid\right.$ dados $)$ é função de $\theta_{2}, \ldots, \theta_{p}$ para cada valor de $\theta_{1}$ fixado.

Seja $\left(\hat{\theta}_{2}, \ldots, \hat{\theta}_{p}\right)$ o vetor de valores dos parâmetros que maximiza $-n h_{\theta_{1}}\left(\theta_{2}, \ldots, \theta_{p}\right)\left(\left(\hat{\theta}_{2}, \ldots, \hat{\theta}_{p}\right)\right.$ é a moda a posteriori $)$ e seja $\sigma=\left\{\operatorname{det}\left(D^{2} h\left(\hat{\theta}_{2}, \ldots, \hat{\theta}_{p}\right)\right)\right\}^{-1 / 2}$ onde $D^{2} h\left(\theta_{2}, \ldots, \theta_{p}\right)$ é a Matriz Hessiana de $\mathrm{h}$ calculada em $\left(\hat{\theta}_{2}, \ldots, \hat{\theta}_{p}\right)$. Então, a aproximação de Laplace para a densidade a posteriori marginal para $\theta_{1}$ é dada por:

$$
\int \exp \left\{-n h_{\theta_{1}}\left(\theta_{2}, \ldots, \theta_{p}\right)\right\} d \theta_{2} \ldots d \theta_{p} \propto(2 \pi)^{p / 2} \sigma e^{-n h\left(\widehat{\theta}_{2}, \ldots, \hat{\theta}_{p}\right)}
$$

Portanto,

$$
\Pi\left(\theta_{1} \mid \text { dados }\right) \propto(2 \pi)^{p / 2} \sigma e^{-n h\left(\widehat{\theta}_{2}, \ldots, \widehat{\theta}_{p}\right)}
$$

Como uma aplicação particular, o Método de Laplace é usado para aproximar a densidade a posteriori marginal do parâmetro de interesse $\theta_{1}, \Pi\left(\theta_{1} \mid\right.$ dados $)$, determinada no Capítulo 3.

Seja a densidade posteriori marginal para $\theta_{1}, \theta_{1} \in\left\{\theta_{1}, \beta: \theta_{1}>0\right.$ e $-\infty<$ $\beta<\infty\}$, dada por:

$$
\Pi\left(\theta_{1} \mid \text { dados }\right)=\int \Pi\left(\theta_{1}, \beta \mid d a d o s\right) d \beta
$$


A densidade $\Pi\left(\theta_{1} \mid\right.$ dados $)$ pode ser reescrita de maneira adequada, tal que:

$$
\Pi\left(\theta_{1} \mid \text { dados }\right)=\int e^{-n h_{\theta_{1}}(\beta)} d \beta
$$

onde $n h_{\theta_{1}}(\beta)=\ln \Pi\left(\theta_{1}, \beta \mid\right.$ dados $)$ é função de $\beta$ para um valor $\theta_{1}$ fixado.

Seja $\widehat{\beta}$ o valor de $\beta$ que maximiza $-n h_{\theta_{1}}(\beta)$ ( $\hat{\beta}$ é a moda da posteriori ) e $\sigma=\left\{h^{\prime \prime}(\hat{\beta})\right\}^{-1 / 2}$. Então, a aproximação de Laplace para a densidade posteriori marginal para $\theta_{1}$ é dada por:

$$
\int \exp \left\{-n h_{\theta_{1}}(\beta)\right\} d \beta \propto \sqrt{2 \pi} \sigma n^{-1 / 2} \exp \{-n h(\beta)\}
$$

Portanto,

$$
\Pi\left(\theta_{1} \mid \text { dados }\right) \propto \sqrt{2 \pi} \sigma n^{-1 / 2} \exp \{-n h(\beta)\}
$$

Especificamente, para a densidade encontrada no Capítulo 3, (3.6), tem-se que:

$$
\Pi\left(\theta_{1} \mid \text { dados }\right) \propto \frac{1}{\theta_{1}^{r+1}} \int_{-\infty}^{\infty} \frac{\prod_{i=1}^{k} V_{i}^{\beta r_{i}}}{V_{1}^{\beta r}} \exp \left\{-\frac{\sum_{i=1}^{k} A_{i} V_{i}^{\beta}}{\theta_{1} V_{1}^{\beta}}\right\} d \beta
$$

Assim,

$$
n h_{\theta_{1}}(\beta)=\beta r V_{1}-\beta \sum_{i=1}^{k} r_{i} \ln V_{i}+\frac{1}{\theta_{1} V_{1}^{\beta}} \sum_{i=1}^{k} A_{i} V_{i}^{\beta}
$$

ou seja,

$$
h_{\theta_{1}}^{\prime \prime}(\widehat{\beta})=\frac{1}{\theta_{1} V_{1}^{\widehat{\beta}}}\left\{\sum_{i=1}^{k} A_{i} V_{i}^{\widehat{\beta}}\left(\ln V_{i}\right)^{2}-\left(\ln V_{1}\right)\left(\sum_{i=1}^{k} A_{i} V_{i}^{\widehat{\beta}}\right)\right\}+\ln V_{1}\left[\sum_{i=1}^{k} r_{i} \ln V_{i}-r \ln V_{1}\right]
$$

Portanto,

$$
\Pi\left(\theta_{1} \mid \text { dados }\right) \propto \frac{1}{\theta_{1}^{r+1}}\left[h^{\prime \prime}(\widehat{\beta})\right]^{-1 / 2} \frac{1}{V_{1}^{r \widehat{\beta}}} \prod_{i=1}^{k} V_{i}^{\widehat{\beta} r_{i}} \exp \left\{-\frac{\sum_{i=1}^{k} A_{i} V_{i}^{\widehat{\beta}}}{\theta_{1} V_{1}^{\widehat{\beta}}}\right\}, \quad \theta_{1} \geq 0
$$

Uma outra aplicação para o Método de Laplace, é a aproximação de integrais para calcular esperanças ou variâncias a posteriori de uma densidade (ver por exemplo, Achcar, 1989) ou ainda, densidades preditivas para observações futuras. 


\section{Apêndice $\mathbf{C}$}

\section{Densidade a Posteriori Marginal para $\delta_{1}=\ln \theta_{1}$ quando $\theta_{1}$ e $\beta$ são desconhecidos, onde os tempos de sobrevivência assumem uma distribuição Exponen- cial} em (3.7).

Assumindo $\beta$ desconhecido, a densidade a posteriori marginal para $\theta_{1}$ é dada

Para a construção de um intervalo de credibilidade para o parâmetro de interesse $\theta_{1}$, podem ser usados métodos numéricos, ou uma transformação de $\theta_{1}$ que leve à distribuições próximas da Normal.

Considere a transformação da variável $\delta_{1}=\ln \theta_{1}$ e a densidade a posteriori conjunta para $\alpha$ e $\beta$ dada em (3.2). Utilizando uma transformação de variáveis, a densidade a posteriori conjunta para $\theta_{1}$ e $\beta$ é determinada.

Seja então a transformação de variáveis:

$$
\left\{\begin{array}{l}
\beta=\beta \\
\delta_{1}=\ln \left(\alpha / V_{1}^{\beta}\right), \text { então, } \alpha=e^{\delta_{1}} V_{1}^{\beta}
\end{array}\right.
$$

A matriz Jacobiana é dada por:

$$
J=\left(\begin{array}{ll}
\partial \beta / \partial \beta & \partial \beta / \partial \delta_{1} \\
\partial \alpha / \partial \beta & \partial \alpha / \partial \delta_{1}
\end{array}\right)
$$

Portanto, $\operatorname{det}(J)=e^{\delta_{1}} V_{1}^{\beta}$.

A densidade a posteriori conjunta para $\delta_{1}$ e $\beta$ é dada por:

$$
\Pi\left(\delta_{1}, \beta \mid \text { dados }\right) \propto \prod_{i=1}^{k} \frac{V_{i}^{\beta r_{i}}}{e^{\delta_{1} r} V_{1}^{\beta r}} \exp \left\{-\frac{\sum_{i=1}^{k} A_{i} V_{i}^{\beta}}{e^{\delta_{1}} V_{1}^{\beta}}\right\}
$$

Integrando a equação acima em relação à $\beta$, a densidade marginal para $\delta_{1}$ 
encontrada é dada por:

$$
\Pi\left(\delta_{1} \mid \text { dados }\right)=\frac{1}{e^{\delta_{1} \tau}} \int_{-\infty}^{\infty} \frac{\prod_{i=1}^{k} V_{i}^{\beta r_{i}}}{V_{1}^{\beta r}} \exp \left\{-\frac{\sum_{i=1}^{k} A_{i} V_{i}^{\beta}}{e^{\delta_{1}} V_{1}^{\beta}}\right\} d \beta
$$

De forma análoga à densidade (3.6), aqui existem dificuldades para resolver analiticamente essa integral. Aplicando o Método de Laplace para aproximação de integrais (ver Apêndice $B), \Pi\left(\delta_{1} \mid d a d o s\right)$ pode ser reescrita da seguinte maneira:

$$
\Pi\left(\delta_{1} \mid \text { dados }\right) \propto \frac{1}{e^{\delta_{1} r}} \int_{-\infty}^{\infty} \exp \left\{-n h_{\delta_{1}}(\beta)\right\} d \beta
$$

onde $n h_{\delta_{1}}(\beta)=\beta r \ln V_{1}-\beta \sum_{i=1}^{k} r_{i} \ln V_{i}+\sum_{i=1}^{k} A_{i} V_{i}^{\beta} / e^{\delta_{1}} V_{1}^{\beta}$. Daí,

$$
\int \exp \{-n h(\beta)\} d \beta \approx \sqrt{2 \pi} \sigma n^{-1 / 2} \exp \{-n h(\widehat{\beta})\}
$$

Assim, a densidade posteriori marginal para $\delta_{1}$, aproximada pelo Método de Laplace, é dada por:

$$
\Pi\left(\delta_{1} \mid \text { dados }\right) \propto \frac{1}{e^{\delta_{1} r}}\left\{h^{\prime \prime}(\widehat{\beta})\right\}^{-1 / 2} \frac{\prod_{i=1}^{k} V_{i}^{\widehat{\beta} r_{i}}}{V_{1}^{\widehat{\beta} r}} \exp \left\{-\frac{\sum_{i=1}^{k} A_{i} V_{i}^{\widehat{\beta}}}{e^{\delta_{1}} V_{1}^{\widehat{\beta} r}}\right\}
$$

Partindo de uma priori de Jeffreys, dada em (3.1), que implica numa priori uniforme em relação à $\delta_{1}$ e $\beta$, a densidade a posteriori conjunta $\Pi\left(\delta_{1}, \beta \mid\right.$ dados $)$ e a função de verossimilhança para $\delta_{1}$ e $\beta$, serão equivalentes.

A função de verossimilhança para $\delta_{1}$ e $\beta$ é dada por:

$$
L\left(\delta_{1}, \beta\right) \propto \prod_{i=1}^{k}\left\{\frac{V_{i}^{\beta r_{i}}}{\left(e^{\delta_{1}} V_{1}^{\beta}\right)^{r_{1}}} \exp \left[-\frac{A_{i} V_{i}^{\beta}}{e^{\delta_{1}} V_{1}^{\beta}}\right]\right\}
$$

ou seja, $l\left(\delta_{1}, \beta\right)=\beta \sum_{i=1}^{k} r_{i} \ln V_{i}-r \delta_{1}-r \beta \ln V_{1}-\sum_{i=1}^{k} A_{i} V_{i}^{\beta} / e^{\delta_{1}} V_{1}^{\beta}$.

Os estimadores de máxima verossimilhança de $\left(\delta_{1}, \beta\right)$ são dados pela resolução das equações:

$$
\left\{\begin{array}{l}
\frac{\partial l\left(\delta_{1}, \beta\right)}{\partial \delta_{1}}=0 \\
\frac{\partial l\left(\delta_{1}, \beta\right)}{\partial \beta}=0
\end{array}\right.
$$


ou seja,

$$
\left\{\begin{array}{cl}
\hat{\delta}_{1} & =\ln \left(\sum_{i=1}^{k} A_{i} V_{i}^{\widehat{\beta}}\right)-\ln r-\hat{\beta} \ln V_{1} \\
\sum_{i=1}^{k} r_{i} \ln V_{1}-r \ln V_{1} & =\left(\sum_{i=1}^{k} A_{i} V_{i}^{\widehat{\beta}} \ln V_{i}-\ln V_{1} \sum_{i=1}^{k} A_{i} V_{i}^{\widehat{\beta}}\right) / V_{1}^{\widehat{\beta}} e^{\widehat{\delta}_{1}}
\end{array}\right.
$$

Usando o resultado (2.2), tem-se que:

$$
\left(\widehat{\delta}_{1}, \hat{\beta}\right) \stackrel{a}{\sim} N\left\{\left(\delta_{1}, \beta\right) ; I^{-1}\left(\hat{\delta}_{1}, \widehat{\beta}\right)\right\}
$$

onde $I(\delta, \beta)$ é a Matriz de Informação de Fisher dada por:

$$
I\left(\delta_{1}, \beta\right)=\left(\begin{array}{lr}
E\left\{-\partial^{2} l / \partial \delta_{1}^{2}\right\} & E\left\{-\partial^{2} l / \partial \delta_{1} \partial \beta\right\} \\
E\left\{-\partial^{2} l / \partial \delta_{1} \partial \beta\right\} & E\left\{-\partial^{2} l / \partial \beta^{2}\right\}
\end{array}\right)
$$

ou seja,

$$
I\left(\delta_{1}, \beta\right)=\left(\begin{array}{cc}
r & r \ln V_{1}-\sum_{i=1}^{k} r_{i} \ln V_{i} \\
r \ln V_{1}-\sum_{i=1}^{k} r_{i} \ln V_{i} & a
\end{array}\right)
$$

onde $a=\sum_{i=1}^{k} r_{i}\left(\ln V_{i}\right)^{2}-2 \ln V_{1} \sum_{i=1}^{k} r_{i} \ln V_{i}+r\left(\ln V_{1}\right)^{2}$ e portanto,

$$
I^{-1}\left(\widehat{\delta}_{1}, \widehat{\beta}\right)=\left(\begin{array}{cc}
\hat{\sigma}_{\delta_{1}}^{2} & \widehat{\sigma}_{\delta_{1}, \beta} \\
\hat{\sigma}_{\beta, \delta 1} & \widehat{\sigma}_{\beta}^{2}
\end{array}\right)
$$

é a Matriz de Variância e Covariância para os parâmetros $\delta_{1}$ e $\beta$.

Como os elementos da Matriz de Informação de Fisher, $I\left(\delta_{1}, \beta\right)$, são constantes, os resultados assintóticos obtidos são precisos (ver por exemplo, Sprott, 1973, 1980).

Observe que

$$
\widehat{\delta}_{1} \stackrel{a}{\sim} N\left\{\delta_{1} ; \hat{\sigma}_{\delta_{1}}^{2}\right\}
$$

Então, um intervalo de credibilidade $100(1-\gamma) \%$ para $\delta_{1}$ é dado por:

$$
i c\left(\delta_{1}\right)=\left[\hat{\delta}_{1}-Z_{\gamma / 2} \hat{\sigma}_{\delta_{1}} ; \hat{\delta}_{1}+Z_{\gamma / 2} \widehat{\sigma}_{\delta_{1}}\right]
$$




\section{Bibliografia}

ABRAMOWITZ, M. ; STEGUN, I.A. Handbook of Mathematical Functions with formulas, graphs and mathematical tables. New York, Dover, 1968

ACHCAR, J.A. Densidades Preditivas em Análise de Sobrevivência In: Simpósio Nacional de Probabilidade e Estatística, 6, Rio de Janeiro, 1984. Atas Rio de Janeiro, IM/UFRJ, 1984 p. 1-7, 1984

ACHCAR, J.A. Reparametrizations and Laplace Approximations for Posteriori Moments in Binomial Models. Revista de Matemática e Estatística, V. 7, p. 73 - 86, 1989

ACHCAR, J.A. Use of predictive densities in quality control with accelerated life tests assuming a power rule model and an exponential distribution. São Carlos, ICMSC - USP, 1990. (Notas do ICMSC - USP, 76)

ACHCAR, J.A. ; BOLFARINE, H. Predictive Densities in Survival Analysis with a Generalized Gamma Regression Model. Revista Brasileira de Probabilidade e Estatistica, V. 2, p. $23-31,1988$

ACHCAR, J.A. ; DIAS, T.C.M. Accelerated tests with an Exponential distribution: a Bayesian approach with the power rule model and type II censored data. São Carlos, ICMSC - USP, 1990. (Notas do ICMSC - USP, 67)

ACHCAR, J.A. ; SMITH, A.F.M. Aspects of Reparametrization in Aproximate Bayesian Inference In: GEISSER, S. ; HODGES, J. S. et al. Bayesian and Likelihood Methods In Statistics and Econometrics. Holanda, Elsevire, 1990. p. 431 - 52. (Statistics in Bayesian Econometrics and Statisticas, 7)

AITCHINSON, J. ; DUNSMORE, I.R. Statistical Prediction Analysis. Cambridge, Cambridge University Press, 1975

BHATTACHARYA, S.K. Bayesian Approach to Life Testing and Reliability Estimation. 
Journal of the American Statistical Association, V. 62, p. 48 - 62, 1967

BHATTACHARYYA, G.K. ; FRIES, A. Inverse Gaussian Regression and Accelerated Life Tests In Proceedings of the Special Topics Meeting on Survival Analysis. In: 138th Meeting of the Institute of Mathematics Statistics. Columbus, Ohio, p. 101 17,1981

BICKEL, P.J ; DOKSUM, K.A. Mathematical Statistics. San Francisco, Holden Day, 1977

BOX, G.E.P. ; TIAO, G.C. Bayesian Inference in Statistical Analysis. New York: Addison - Wesley, 1973

COHEN, A.C. Maximum Likelihood Estimation in the Weibull Distribution Basead on Complete and Censored Samples. Technometrics, V. 7, N. 4, p. 579-88, 1965

COX, D.R. ; REID, N. Parameter Orthogonality and Approximate Conditional Inference. Journal Royal Statistical Society, V. 49, N. 1, p. 1 - 39, 1987

EPSTEIN, B. ; SOBEL, M. Life Testing. Journal of the American Statistical Association, $V .48, N .263$, p. $486-502,1953$

GEISSER, S. Aspects of the Preditive and Estimative Approachs in the Determination of Probabibilities. Biometrics Supplement: Current Topics in Biostatistics and Epidemiology, p. $75-85,1982$

KAPLAN, E.L. ; MEIER, P. Nonparametric Estimation from Incomplete Observations. Journal of the American Statistical Association, V. 53, N. 282, p. 457-81, 1958

KLEIN, J.P. ; BASU, A.P. Accelerated Life Testes Under Competing Weibull Causes of Failure. University of Missouri - Columbia, Technical Reporter N. 103, 1981

KLEIN, J.P. ; BASU, A.P. Weibull Accelerated Life Tests when there are Competing Causes of Failure. Communications in Statistics - Theory ans Methods, A10(20), p. 2073 - 100, 1981a

LAWLESS, J.F. Statistical Models and Methods for Lifetime Data. New York, Willey, 1982

LEVENBACH, G.J. Accelerated Life Testing of Capacitors. IRE Transactions, V. 10, p. $9-20,1957$

MANN, N.R. ; SCHAFFER, R.E. ; SINGPURWALLA, N.D. Methods for Statistical 
Analysis of Reliability and Life Data. New York, Wiley, 1974

MILLER, R.G. Survival Analysis. New York, Wiley, 1981

MOOD, A.M. ; GRAYBILL, F.A. ; BOES, D.C. Introduction to the Theory of Statistical. Englewood Cliffs, N.J., McGraw - Hill, 1974

NELSON, W.B. Graphical Analysis of Accelerated Life Test Data with the Inverse Power Law Model. IEEE Transactions on Reliability, V. R-21, N. 1, p. 2 - 11, 1972

SINGPURWALLA, N.D. Inference from Accelerated Life Tests When Observations are Obtained from Censored Samples. Technometrics, V. 13, N. 1, p. $161-70,1971$

PRESS, S.J. Bayesian Statistics: Principles, Models and Applications. New York, Willey, 1988

SPROTT, D.A. Maximum Likelihood in Small Samples: Estimation in the Presence of Nuisance Parameters. Biometrika, V. 67, p. $515-23,1980$

SPROTT. D.A. Normal Likelihoods and Relation to a Large Sample Theory of Estimations. Biometrika, V. 60, p. $457-65,1973$

SPROTT, D.A. ; KALBFLEISH. J.D. Examples of Likelihoods and Comparison with point Estimates and Large Samples Approximations. Journal of the American Statistical Association, V. 64, N. 326, p. 468 - 84, 1968

TIERNEY, L. ; KADANE, J.B. Accurate Approximations for Posteriori Moments and Marginal Densities. Journal of the American Statistical Association, V. 81, N. 393, p. $82-6,1986$

TIERNEY, L. ; KASS, R.E. ; KADANE, J.B. Fully Exponential Laplace Approximations to Expectations and Variances of Nonpositive Functions. Journal of the American Statistical Association. V. 84, N. 407 p. 710 - 16, 1989

ZACKS, S. The Theory of Statistical Inference. New York, Willey, 1971

ZELEN, M. Factorial Experiments in Life Testing. Technometrics, V. 1, N. 3, p. 269 $-288,1959$ 